\title{
Staggered Chiral Perturbation Theory at Next-to-Leading Order
}

\author{
Stephen R. Sharpe* and Ruth S. Van de Wateli \\ Physics Department, University of Washington, Seattle, WA 98195-1560
}

(Dated: November 12, 2018)

\begin{abstract}
We study taste and Euclidean rotational symmetry violation for staggered fermions at nonzero lattice spacing using staggered chiral perturbation theory. We extend the staggered chiral Lagrangian to $\mathcal{O}\left(a^{2} p^{2}\right), \mathcal{O}\left(a^{4}\right)$ and $\mathcal{O}\left(a^{2} m\right)$, the orders necessary for a full next-to-leading order calculation of pseudo-Goldstone boson masses and decay constants including analytic terms. We then calculate a number of $S O(4)$ taste-breaking quantities, which involve only a small subset of these NLO operators. We predict relationships between $S O(4)$ taste-breaking splittings in masses, pseudoscalar decay constants, and dispersion relations. We also find predictions for a few quantities that are not $S O(4)$ breaking. All these results hold also for theories in which the fourth-root of the fermionic determinant is taken to reduce the number of quark tastes; testing them will therefore provide evidence for or against the validity of this trick.
\end{abstract}

\section{INTRODUCTION}

Simulations of lattice QCD using staggered fermions [1] are able, at present, to reach significantly smaller dynamical quark masses than those using other fermion discretizations [2, 3]. This computational advantage is due in part to the axial $U(1)$ symmetry remaining at nonzero lattice spacing, which protects the quark mass from additive renormalization. The primary disadvantage of staggered fermions is that each continuum flavor comes in four tastes. In the continuum limit, the tastes become degenerate and one can formally remove the extra tastes by taking the

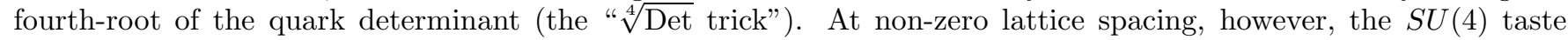
symmetry is broken by discretization errors of $\mathcal{O}\left(a^{2}\right)$, where $a$ is the lattice spacing. These errors are numerically significant in present simulations [4]. This has two related implications. First, since one must, in practice, take the fourth-root of the determinant before taking the continuum limit, the resulting underlying quark action is likely non-local. It is thus not guaranteed to lie in the same universality class as QCD, even if the non-local part is, in some sense, of $\mathcal{O}\left(a^{2}\right)$ and thus small. ${ }^{1}$ Second, assuming that the continuum limit is correct, it is necessary to perform a combined continuum and chiral extrapolation taking into account taste-breaking effects of $\mathcal{O}\left(a^{2}\right)$ [7].

The first issue is clearly more fundamental, but also more difficult to address. To date, support for the use of the $\sqrt[4]{\text { Det }}$ trick comes from an accumulation of numerical or indirect evidence: the accurate agreement of lattice and experimental results for all "gold-plated" quantities that have been calculated [3]; the success of fits of the light pseudoscalar meson masses and decay constants to the predictions of chiral perturbation theory including the $\mathcal{O}\left(a^{2}\right)$ taste-breaking errors [4]; the near four-fold degeneracy of eigenvalues of the staggered Dirac operator and its ability to correctly measure the topology of the gauge configurations $[8,9]$; and the results of analytic studies of the time continuum limit [10, 11]. None of these tests purports to be conclusive. Nevertheless, in the absence of a more theoretical approach, it is important to have further numerical tests. This is one of the motivations for the present work.

Our major focus, however, is on the further development of chiral perturbation theory for staggered fermions including discretization errors. This "staggered chiral perturbation theory" (or S $\chi \mathrm{PT}$ for short) is the theoretical tool which determines the functional forms needed to do the combined continuum and chiral extrapolations incorporating taste violations. The development of $\mathrm{S} \chi \mathrm{PT}$ for staggered fermions without the $\sqrt[4]{\text { Det }}$ trick, i.e. with four tastes per flavor, is a standard application of the methods of effective field theory [12, 13]. In order to apply S $\chi \mathrm{PT}$ to simulations done with the $\sqrt[4]{\text { Det }}$ trick, however, one must alter the $\chi \mathrm{PT}$ Feynman rules by hand (or alternatively use a variant of the replica trick [14] ), so there is no underlying "staggered chiral Lagrangian" which can reproduce the Feynman rules 13. Nevertheless, if the resulting expressions describe the outcomes of numerical simulations done using the

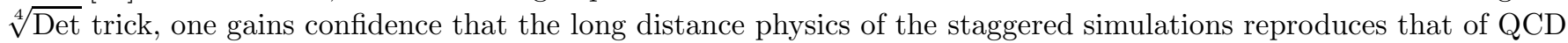
in the continuum limit. Indeed, the expressions in $\mathrm{S} \chi \mathrm{PT}$ go over to those of standard $\mathrm{QCD} \chi \mathrm{PT}$ in the continuum limit, and give an explicit example of how the effect of the $\sqrt[4]{\text { Det }}$ trick can vanish smoothly.

\footnotetext{
*Electronic address: sharpe@phys.washington.edu

$\dagger$ Electronic address: ruthv@u.washington.edu

${ }^{1}$ For summaries of this and related problems with the $\sqrt[4]{\text { Det }}$ trick see Refs. [5, 6].
} 
We consider here only $\mathrm{S} \chi \mathrm{PT}$ applied to the properties of pseudo-Goldstone bosons (PGBs). ${ }^{2} \mathrm{~S} \chi \mathrm{PT}$ is a combined expansion in powers of the quark masses and lattice spacing, in which the usual power counting is $m \sim a^{2}$. The leading order (LO) correction to continuum $\chi \mathrm{PT}$ is the $\mathcal{O}\left(a^{2}\right)$ potential, which was determined for a single flavor of staggered fermion in Ref. 12]. This potential retains an $S O(4)$ subgroup of the continuum $S U(4)$ taste symmetry. Since this subgroup is larger than the discrete lattice symmetry group, the masses of the PGBs in certain lattice irreps are predicted, at LO, to be degenerate. Reference [13] generalized the potential to multiple staggered flavors, and showed that the degeneracies remain. These predictions of S $\chi \mathrm{PT}$ work very well; both in old quenched results [15, 16, 17] and in recent dynamical simulations (using the $\sqrt[4]{\mathrm{Det}}$ trick) [4]. This success gives one confidence in the utility of S $\chi \mathrm{PT}$.

It is well known from continuum $\chi \mathrm{PT}$ that practical applications to the properties of the physical PGB mesons requires one to work at least to next-to-leading order (NLO). The same is true of chiral extrapolations of lattice data [18]. Thus for $\mathrm{S} \chi \mathrm{PT}$ to be a practical tool for guiding extrapolations, it must be extended beyond LO. Important first steps were taken in Refs. [13] and [19], where the one-loop contributions to the mass and decay constant of the lattice Goldstone boson (that with taste $\xi_{5}$ ) were calculated. These forms, and in particular the taste-breaking built into them, have proved essential to describe the numerical results [7]. Conversely, the success of the fits provides more evidence for the applicability of $\mathrm{S} \chi \mathrm{PT}$, and the lack of problems introduced by the $\sqrt[4]{\text { Det }}$ trick.

In this paper we continue the extension of S $\chi \mathrm{PT}$ to NLO by determining all the operators proportional to $a^{2} p^{2}$, $a^{2} m$ and $a^{4}$, including source terms for left- and right-handed currents and for scalar and pseudoscalar densities. These operators give the analytic NLO contributions that incorporate the effects of discretization. Each is multiplied by a different, unknown low energy coefficient (LEC). The full NLO result is obtained by adding their contributions to those from one-loop diagrams and from the continuum NLO operators.

In continuum $\chi \mathrm{PT}$ there are of order ten NLO operators, and it is possible to consider enough physical quantities to determine their coefficients and make predictions. In $\mathrm{S} \chi \mathrm{PT}$ we find that there are an order of magnitude more NLO operators. While, in principle, there is a corresponding increase in the number of physical quantities that can be calculated (e.g. scattering amplitudes with many choices of external tastes), in practice it will be difficult to carry through a NLO program along the lines of that in the continuum.

Nevertheless, our enumeration of operators is not a purely academic exercise, for there is one set of quantities for which very few operators contribute, and a number of new predictions can be made. These are quantities which are non-vanishing only because the $S O(4)$ taste symmetry is broken. Since this symmetry is preserved by the LO Lagrangian, it is also preserved by the one-loop contributions, as these involve LO vertices. Thus the only source of $S O$ (4) breaking is the NLO operators. Furthermore, we find that for pion masses and pseudoscalar decay constants (i.e. the vacuum to pion matrix element of the pseudoscalar density), the only contribution to $S O(4)$ breaking comes from the operators proportional to $a^{2} p^{2}$, and that there are few enough of these that we can make several predictions. These are valid only at NLO, being violated by higher order terms. Comparison of these S $\chi \mathrm{PT}$ predictions with lattice data numerically tests the $\sqrt[4]{\text { Det }}$ trick.

Discussions of staggered fermions can quickly become rather technical, so in an attempt to keep the main text of this paper accessible, we relegate most of the details of operator enumeration to an appendix. The precise organization is as follows. In Sections \and WII we review the construction of the continuum quark-level effective staggered action and leading order staggered chiral perturbation theory, respectively. In Section IV] we discuss the symmetry-breaking pattern exhibited by the $\mathcal{O}\left(a^{2} p^{2}\right), \mathcal{O}\left(a^{4}\right)$, and $\mathcal{O}\left(a^{2} m\right)$ operators, and outline the general consequences for various PGB properties. Our specific predictions for relations between $S O(4)$ breaking in masses, dispersion relations and pseudoscalar decay constants are presented and explained in Section $\nabla$ We conclude in Section VI We determine the NLO staggered operators in Appendix $\mathrm{A}$ and collect them in the tables of Appendix B]

\section{CONTINUUM EFFECTIVE LAGRANGIAN FOR STAGGERED QUARKS}

The construction of the chiral Lagrangian including discretization errors proceeds in two steps [20]. One first determines the continuum effective Lagrangian for quarks with momenta much smaller than the lattice cut-off $1 / a$. The dominant power-law dependence on the lattice spacing is thereby made explicit. In the second step one maps the continuum quark-level Lagrangian onto an effective chiral Lagrangian. In this section we briefly review the first step for staggered fermions, including in the effective Lagrangian the leading discretization effects, which are proportional

\footnotetext{
2 There is a potential ambiguity in this terminology. We use the term PGB to refer to all pseudoscalar mesons (and fermions, once we consider partially quenched theories) whose masses vanish in the combined chiral and continuum limits. This includes the pion whose mass vanishes in the chiral limit even at non-zero lattice spacing, which we refer to as the "lattice Goldstone" pion, as well as all the other light pseudoscalars with different tastes.
} 
to $a^{2}$. This Lagrangian was determined for a single flavor in Ref. [12], and generalized to multiple flavors in Ref. [13]. While we obtain no new results here, we attempt to clarify aspects of the discussion in these papers.

The construction of the effective quark Lagrangian follows the standard method developed by Symanzik [21], in which one determines all local continuum operators of dimension up to and including six that are invariant under the lattice symmetry group. Given the complexity of the symmetry group for staggered fermions [22], a simple way to perform this enumeration is to first write down allowed lattice operators of dimension up to six, and then match them onto continuum operators. The first step utilizes all of the lattice symmetries, including translations. It is discussed in detail in Appendix A of Ref. [12]. The second step uses only transformations that map the $2^{4}$ hypercube onto itself, which are related to spin and taste transformations in the continuum 23. As is well known, there are no dimension 5 operators 24, 25]. At dimension 6 one finds purely gluonic operators, fermion bilinears and four-fermion operators. These operators were enumerated in Refs. [26] and [12].

Neither the gluonic operators nor the fermion bilinears break the continuum taste symmetry because they do not contain any taste matrices. Since our major focus is taste symmetry breaking, we do not reproduce these operators here. They do, however, break the continuum $S O(4)$ rotation symmetry down to its hypercubic subgroup, $S W_{4}$, and we discuss this issue briefly in Appendix A

$S U(4)$ taste symmetry is broken by the four-fermion operators. We first recall the results for a single flavor and then generalize to multiple flavors. We write quark bilinears using the direct-product (spin $\otimes$ taste) notation of Refs. 27, 28, 29]. ${ }^{3}$ Each four fermion operator in the effective Lagrangian turns out to be a product of two bilinears with the same spin and taste. Thus one can use a compact notation to represent them. Following Refs. [12, 29] we write the pair of spin matrices in the two bilinears as

$$
S \equiv 1 \otimes 1, \quad P \equiv \gamma_{5} \otimes \gamma_{5}, \quad V \equiv \sum_{\mu} \gamma_{\mu} \otimes \gamma_{\mu}, \quad A \equiv \sum_{\mu} \gamma_{\mu 5} \otimes \gamma_{5 \mu}, \quad T \equiv \sum_{\mu<\nu} \gamma_{\mu \nu} \otimes \gamma_{\nu \mu}
$$

using the definitions $\gamma_{\mu \nu} \equiv \gamma_{\mu} \gamma_{\nu}$ and $\gamma_{\mu 5} \equiv \gamma_{\mu} \gamma_{5}$. The pair of taste matrices are denoted in the same manner with $\gamma_{\mu} \rightarrow \xi_{\mu}$. The four fermion operator is then denoted by the spin and taste matrices that it contains. For example

$$
[A \times T] \equiv \sum_{\mu} \sum_{\nu<\rho} \bar{Q}\left(\gamma_{\mu 5} \otimes \xi_{\nu \rho}\right) Q \bar{Q}\left(\gamma_{5 \mu} \otimes \xi_{\rho \nu}\right) Q
$$

where $Q, \bar{Q}$ are Dirac fields with spinor and taste indices. We stress that $Q, \bar{Q}$ are at this stage continuum fields, and that the two $Q$ 's and two $\bar{Q}$ 's in this operator reside at the same position in Euclidean space.

The fields $Q, \bar{Q}$ also have color indices, which can be contracted in (2) either within each bilinear or between them. These two color contractions are related by Fierz transformations. In Ref. [12], these transformations were used to select a basis in which all operators had the same type of color contraction. Here we proceed differently, following Ref. [13]. We keep both color contractions, and use the notation such as $[A \times T]$ to implicitly refer to both. This is a sensible notation because, when we map these operators into the chiral Lagrangian, the choice of color contraction is irrelevant: the two operators map onto the same chiral operators. Using this notation allows us to shorten the list of operators, since by Fierz transformations we can always bring the operators into a form in which both bilinears have "odd distance" (see Ref. 12] for an explanation of this terminology). For example, the operator $\{[S \times S]-[P \times P]\}$, which consists of two bilinears both of distance zero, can be transformed into a linear combination of operators with odd-distance bilinears.

We now list the resulting four-fermion operators. They can be divided into two types based on how badly they break taste symmetry. Twelve have their spin and taste indices contracted separately, as in the example of (2), and are therefore rotationally-invariant:

$$
\begin{aligned}
\mathcal{L}_{6}^{\mathrm{FF}(\mathrm{A}) \sim} & {[S \times A]+[S \times V]+[A \times S]+[V \times S]+[P \times V]+[P \times A]+[V \times P]+[A \times P]+} \\
& {[T \times V]+[T \times A]+[V \times T]+[A \times T] . }
\end{aligned}
$$

Each operator has a unique coefficient, which we do not show, that is proportional to $a^{2}$, but also depends on $g(a)^{2}$ and $\ln (a)$. Because the indices on the taste matrices are contracted between bilinears, these operators preserve an $S O(4)$ subgroup of the full $S U(4)$ taste symmetry. The four remaining four fermion operators have spin and taste indices contracted together:

$$
\mathcal{L}_{6}^{\mathrm{FF}(\mathrm{B})} \sim\left[T_{\mu} \times V_{\mu}\right]+\left[T_{\mu} \times A_{\mu}\right]+\left[V_{\mu} \times T_{\mu}\right]+\left[A_{\mu} \times T_{\mu}\right] .
$$

\footnotetext{
${ }^{3}$ In the standard staggered convention, the spin matrices, $\gamma_{S}$, are Euclidean gamma matrices, while the taste matrices, $\xi_{T}$, are complexconjugated Euclidean gamma matrices.
} 
[The precise meaning of this notation can be seen from the example in (A19).] They are therefore only invariant under certain combined spin and taste rotations, and break the full spin-taste symmetry down to the discrete subgroup respected by the lattice theory, $\Gamma_{4} \rtimes S W_{4}$ [30]. In other words, these "FF(B)" operators break the continuum symmetry maximally.

One advantage of this basis is that the $U(1)_{A}$ symmetry of the lattice theory (which is exact in the chiral limit) is manifest. Under this symmetry

$$
Q \longrightarrow \exp \left(i \alpha\left[\gamma_{5} \otimes \xi_{5}\right]\right) Q, \quad \bar{Q} \longrightarrow \bar{Q} \exp \left(i \alpha\left[\gamma_{5} \otimes \xi_{5}\right]\right)
$$

and each of the operators in (3) and (4) is separately invariant. Another advantage is that there are fewer operators to consider; Ref. [12] had an additional six $F F(A)$ and two $F F(B)$ operators.

Thus far we have only considered the single flavor theory. We now recall the generalization to $n>1$ flavors 13 . The quark field picks up a flavor index, $Q \rightarrow Q_{i}$, and bilinear operators generalize straightforwardly, e.g. $m \bar{Q} Q \rightarrow$ $\sum_{i} m_{i} \bar{Q}_{i} Q_{i}$. The generalization of the four-fermion operators is more subtle. Since these operators are the result of hard gluon exchange, and gluons do not change flavor, they can be written in a form containing flavor diagonal bilinears, i.e.

$$
\sum_{i, j} \bar{Q}_{i}\left(\gamma_{S} \otimes \xi_{T}\right) Q_{i} \bar{Q}_{j}\left(\gamma_{S} \otimes \xi_{T}\right) Q_{j}
$$

Of course we could Fierz transform such an operator to bring it into a "flavor-mixed" form, but it is advantageous not to do so. This is because, in the unmixed basis (6), each bilinear must have odd distance in order to be invariant under the lattice axial symmetry, as discussed in the following paragraph. Thus the spin-taste structure of the $F F(A)$ and $F F(B)$ four-fermion operators remains as in (3) and (4), respectively, but the flavor structure is now given by (6). As for the single flavor case, there are two possible color contractions of each operator, but we do not show these explicitly as they play no role when we map the operators into the chiral Lagrangian.

We emphasize that the result that only odd-distance bilinears appear in the flavor unmixed basis follows from symmetries alone. The discussion of gluon exchanges is given only to provide motivation for considering the flavor unmixed basis, but is not strictly necessary. Since the four-fermion operators remain in the chiral limit, they must be invariant under the symmetries of the lattice theory in that limit. These include $U(n)$ axial transformations, which act as in (15), except that $\alpha$ is now an Hermitian $n \times n$ flavor matrix, and $Q$ a flavor vector. ${ }^{4}$ In the lattice theory, this corresponds to rotating even and odd sites by opposite $U(n)$ transformations. It is easy to check that the operators in (3) and (4) are invariant under $U(n)$ transformations if they are composed of flavor diagonal bilinears. On the other hand, compound operators with spin-taste structure such as $[S \times S]-[P \times P]$ are not invariant for more than one flavor. We stress that it is not sufficient to consider only $U(1)^{n}$ transformations (in which each flavor is rotated independently), since this does not rule out compound operators of the form

$$
\sum_{i}\left[\bar{Q}_{i}\left(\gamma_{I} \otimes \xi_{I}\right) Q_{i} \bar{Q}_{i}\left(\gamma_{I} \otimes \xi_{I}\right) Q_{i}-\bar{Q}_{i}\left(\gamma_{5} \otimes \xi_{5}\right) Q_{i} \bar{Q}_{i}\left(\gamma_{5} \otimes \xi_{5}\right) Q_{i}\right]
$$

These are only ruled out by their lack of invariance under general $U(n)$ transformations.

Finally, we comment briefly on operators of yet higher dimension. Since we aim to determine operators in $\mathrm{S} \chi \mathrm{PT}$ proportional to $a^{4}$, one might have thought that we need to enumerate all dimension 8 operators in the continuum effective theory (dimension 7 operators are forbidden by the axial symmetry). Fortunately this is not the case. The naive argument for this is that the dimension 6 operators break the continuum symmetries down to the lattice subgroup, and so dimension 8 operators break no further symmetries. Since it is the symmetries that are used to map to the chiral Lagrangian, the dimension 8 operators are not needed. This argument is, however, incomplete. What matters in addition is the number of taste matrices with correlated indices in the quark-level operators. If there are more such taste matrices, then one can build different operators in the chiral Lagrangian. We explain this point in Appendix A where in Section A3 we show that certain operators in the chiral Lagrangian do not arise until $\mathcal{O}\left(a^{8}\right)$, since they require four tensor taste matrices with fully correlated indices, and this does not occur unless one has an eight-quark operator. Now, the dimension 6 operators have at most two taste matrices, as exemplified by $[A \times T]$ in (2). Moving to dimension 8 adds further derivatives or gluon fields, but no further bilinears, and thus no more taste matrices. For this reason we do not need to enumerate the dimension 8 operators.

\footnotetext{
4 These transformations do not form a group for $n>1$, but this is not important for our considerations.
} 


\section{CHIRAL LAGRANGIAN FOR STAGGERED QUARKS AT LEADING ORDER}

In this section we review the mapping of the continuum quark effective Lagrangian into the effective chiral Lagrangian, working at leading order in our joint our expansion about the chiral and continuum limits. This was done for a single staggered flavor in Ref. [12], and generalized to multiple flavors in Refs. [13, 31].

If there are $n$ flavors of staggered fermions, then, in the combined chiral-continuum limit, the theory possesses an $S U(4 n)_{L} \times S U(4 n)_{R}$ chiral symmetry. We assume that, as in QCD, this breaks spontaneously down to $S U(4 n)_{V}{ }^{5}$ This symmetry breaking pattern leads to $16 n^{2}-1$ pseudo-Goldstone bosons. We can collect these in the usual way into an $S U(4 n)$ matrix:

$$
\Sigma=\exp (i \Phi / f)
$$

where $\Phi$ is a traceless $4 n \times 4 n$ matrix:

$$
\Phi=\left(\begin{array}{cccc}
U & \pi^{+} & K^{+} & \ldots \\
\pi^{-} & D & K^{0} & \cdots \\
K^{-} & \frac{K^{0}}{} & S & \cdots \\
\vdots & \vdots & \vdots & \ddots
\end{array}\right)
$$

with $4 \times 4$ submatrices:

$$
U=\sum_{a=1}^{16} U_{a} T_{a}
$$

and so forth. The pion decay constant, $f$, is normalized such that $f_{\pi} \approx 132 \mathrm{MeV}$. We choose to express the $S U(4)$ generators in the following Hermitian basis:

$$
T_{a}=\left\{\xi_{5}, i \xi_{\mu 5}, i \xi_{\mu \nu}, \xi_{\mu}, \xi_{I}\right\}
$$

where $\xi_{I}$ is just the $4 \times 4$ identity matrix. It is important to retain the taste singlet meson, $U_{I} \propto \operatorname{tr}(U)$, because with $n$ flavors (and thus $4 n$ tastes), only the overall $S U(4 n)$ singlet decouples. Under an $S U(4 n)_{L} \times S U(4 n)_{R}$ chiral symmetry transformation, $\Sigma$ transforms as

$$
\Sigma \rightarrow L \Sigma R^{\dagger}
$$

where $L \in S U(4 n)_{L}$ and $R \in S U(4 n)_{R}$.

We use the following power-counting scheme when determining the staggered chiral Lagrangian:

$$
p^{2} / \Lambda_{Q C D}^{2} \approx m / \Lambda_{Q C D} \approx a^{2} \Lambda_{Q C D}^{2},
$$

which is consistent with parameters of current simulations. The lowest order Lagrangian, which is of $\mathcal{O}\left(p^{2}, m, a^{2}\right)$, is

$$
\mathcal{L}_{\chi}=\frac{f^{2}}{8} \operatorname{Tr}\left(\partial_{\mu} \Sigma \partial_{\mu} \Sigma^{\dagger}\right)-\frac{1}{4} \mu f^{2} \operatorname{Tr}\left(\mathcal{M} \Sigma+\mathcal{M} \Sigma^{\dagger}\right)+a^{2} \mathcal{V}
$$

where $\mathcal{M}$ is the quark mass matrix:

$$
\mathcal{M}=\left(\begin{array}{cccc}
m_{u} I & 0 & 0 & \cdots \\
0 & m_{d} I & 0 & \cdots \\
0 & 0 & m_{s} I & \cdots \\
\vdots & \vdots & \vdots & \ddots
\end{array}\right)
$$

\footnotetext{
${ }^{5}$ We do not need to worry about a possible change in the dynamics for large numbers of flavors, e.g. the loss of asymptotic freedom, because practical applications always involve three or less light dynamical flavors, for the which the chiral symmetry breaking pattern of QCD should apply. The reduction from $4 n$ quarks to 2-3 sea quarks is accomplished by partial quenching, as explained at the end of this section. The theoretical status of symmetry breaking in partially quenched theories has been discussed in Ref. [32].
} 
and $\mathcal{V}$ is the taste-breaking potential resulting from the four-fermion operators in the quark effective action. Note that $\operatorname{Tr}$ is a full $4 n \times 4 n$ trace in both flavor and taste space. The dimensionful constant, $\mu$, is of $\mathcal{O}\left(\Lambda_{Q C D}\right)$, and is defined such that the PGB mass is given at LO by

$$
\left(m_{\pi}^{2}\right)_{\mathrm{LO}}=2 \mu \frac{m_{i}+m_{j}}{2}+a^{2} \Delta_{F} .
$$

The labels $i$ and $j$ indicate the flavors of quarks in the PGB, here assumed to be different, while the splitting, $\Delta_{F}$, depends on the taste.

The mapping of the four-fermion operators enumerated in the previous section into the mesonic operators in $\mathcal{V}$ is done by treating the taste matrices as spurions. Having worked out the implications of the symmetry under axial $U(n)$ transformations at the quark level, no further subtleties arise in the mapping. The method of Ref. [12], generalized to multiple flavors [13], can be used. A key result is that only the $F F(A)$ operators, i.e. those in (31), contribute to $\mathcal{V}$, so the potential has a larger symmetry than the underlying lattice theory. We give a brief recapitulation of the determination of the form of $\mathcal{V}$ at the end of Secs. $\mathrm{A} 2 \mathrm{a}$ and $\mathrm{A} 2 \mathrm{~b}$ as a byproduct of our extension of the methodology to NLO. In the notation of Ref. [13], the result is

$$
\mathcal{V}=\mathcal{U}+\mathcal{U}^{\prime}
$$

where

$$
\begin{aligned}
-\mathcal{U} \equiv \sum_{k} C_{k} \mathcal{O}_{k}= & C_{1} \operatorname{Tr}\left(\xi_{5}^{(n)} \Sigma \xi_{5}^{(n)} \Sigma^{\dagger}\right) \\
& +C_{3} \frac{1}{2} \sum_{\nu}\left[\operatorname{Tr}\left(\xi_{\nu}^{(n)} \Sigma \xi_{\nu}^{(n)} \Sigma\right)+\text { h.c. }\right] \\
& +C_{4} \frac{1}{2} \sum_{\nu}\left[\operatorname{Tr}\left(\xi_{\nu 5}^{(n)} \Sigma \xi_{5 \nu}^{(n)} \Sigma\right)+\text { h.c. }\right] \\
& +C_{6} \sum_{\mu<\nu} \operatorname{Tr}\left(\xi_{\mu \nu}^{(n)} \Sigma \xi_{\nu \mu}^{(n)} \Sigma^{\dagger}\right)
\end{aligned}
$$

and

$$
\begin{aligned}
-\mathcal{U}^{\prime} \equiv \sum_{k^{\prime}} C_{k^{\prime}} \mathcal{O}_{k^{\prime}}= & C_{2 V} \frac{1}{4} \sum_{\nu}\left[\operatorname{Tr}\left(\xi_{\nu}^{(n)} \Sigma\right) \operatorname{Tr}\left(\xi_{\nu}^{(n)} \Sigma\right)+h . c .\right] \\
& +C_{2 A} \frac{1}{4} \sum_{\nu}\left[\operatorname{Tr}\left(\xi_{\nu 5}^{(n)} \Sigma\right) \operatorname{Tr}\left(\xi_{5 \nu}^{(n)} \Sigma\right)+h . c .\right] \\
& +C_{5 V} \frac{1}{2} \sum_{\nu}\left[\operatorname{Tr}\left(\xi_{\nu}^{(n)} \Sigma\right) \operatorname{Tr}\left(\xi_{\nu}^{(n)} \Sigma^{\dagger}\right)\right] \\
& +C_{5 A} \frac{1}{2} \sum_{\nu}\left[\operatorname{Tr}\left(\xi_{\nu 5}^{(n)} \Sigma\right) \operatorname{Tr}\left(\xi_{5 \nu}^{(n)} \Sigma^{\dagger}\right)\right],
\end{aligned}
$$

Here $\xi_{T}^{(n)}$ is the $4 n \times 4 n$ generalization of the taste-matrix:

$$
\xi_{T}^{(n)}=\left(\begin{array}{cccc}
\xi_{T} & 0 & 0 & \cdots \\
0 & \xi_{T} & 0 & \cdots \\
0 & 0 & \xi_{T} & \cdots \\
\vdots & \vdots & \vdots & \ddots
\end{array}\right),
$$

with $\xi_{T}$ the ordinary $4 \times 4$ taste matrix. This flavor diagonal form mirrors that of the underlying quark operators, (6i).

The potential is both rotationally and $S O(4)$ taste symmetric. Therefore the PGB sector respects a larger symmetry than the lattice theory at $\mathcal{O}\left(a^{2}\right)$. Although the 16 PGB masses (for a given choice of constituent quark flavors) are no longer degenerate as in the continuum, they split into five degenerate groups according to $S O(4)$ representations having tastes $\xi_{5}, \xi_{A}, \xi_{T}, \xi_{V}$, and $\xi_{I}$. The taste $\xi_{5}$ PGB is the lattice Goldstone boson and receives no mass correction from $\mathcal{V}$ because of the exact axial symmetry. Thus $\Delta_{F}$ in (16) in fact only depends on the $S O(4)$ representation of the particular taste. 
The potential can be simplified for a single flavor using Fierz transformations. In particular, all double-trace operators can be transformed into operators with only single traces. This single trace basis is that used in Ref. 12]. Such transformations, however, are not useful for multiple flavors because they do not maintain the flavor diagonal form of $\xi_{T}^{(n)}$. Indeed, as realized in Ref. 13], the double trace operators of $\mathcal{U}^{\prime}$ give rise to a previously unnoticed effect, namely the presence of hairpin diagrams (quark disconnected contractions) for flavor singlet mesons with non-trivial taste. In the LO potential, these occur only for vector and axial tastes, but, as we show below, at NLO they arise for all tastes.

Given the importance of the axial $U(n)$ transformations in restricting the form of the underlying quark operators, as explained in the previous section, it is worthwhile discussing these transformations at the mesonic level. They are simply $S U(4 n)_{A}$ transformations, acting on $\Sigma$ as in (12), with $L=R^{\dagger}=\exp \left(i \alpha \otimes \xi_{5}\right)$. Here, as in the previous section, $\alpha$ is an Hermitian $n \times n$ matrix acting in flavor space, while $\xi_{5}$ acts in taste space. Using the commutation relations among the taste matrices it is easy to show that

$$
\begin{aligned}
L \xi_{\mu}^{(n)} L & =\xi_{\mu}^{(n)}, \quad L \xi_{\mu 5}^{(n)} L=\xi_{\mu 5}^{(n)} \\
L \xi_{5}^{(n)} L^{\dagger} & =\xi_{5}^{(n)}, \quad L \xi_{\mu \nu}^{(n)} L^{\dagger}=\xi_{\mu \nu}^{(n)}
\end{aligned}
$$

It then follows immediately that all the operators in the potential $\mathcal{V}$ are invariant under these transformations.

We conclude by generalizing the results to partially quenched (PQ) theories, since partial quenching is often used in practical simulations. Following Ref. [33], one introduces valence quark flavors with ghost partners, each coming

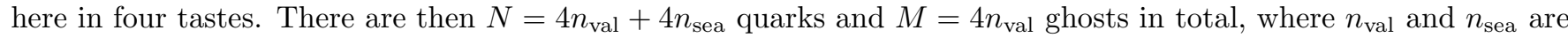
the number of valence and sea flavors, respectively. Note that the unquenched sea-quark sector lies within the PQ theory, so one loses no generality by considering the larger theory 34].

As noted in Ref. [13], the form of the chiral Lagrangian in the PQ theory is unchanged, except that the chiral symmetry group becomes $S U(N \mid M)_{L} \times S U(N \mid M)_{R}$, traces are replaced by supertraces, and the PGB matrix is enlarged. For example, in a theory with two valence quarks ( $x$ and $y$ ) and three sea quarks $(u, d$ and $s)$, the PGB and mass matrices become (schematically)

$$
\Phi=\left(\begin{array}{ccccccc}
X & P^{+} & \ldots & \ldots & \ldots & \ldots & \ldots \\
P^{-} & Y & \ldots & \ldots & \ldots & \ldots & \ldots \\
\vdots & \vdots & U & \pi^{+} & K^{+} & \ldots & \ldots \\
\vdots & \vdots & \pi^{-} & D & K^{0} & \ldots & \ldots \\
\vdots & \vdots & K^{-} & K^{0} & S & \ldots & \ldots \\
\vdots & \vdots & \vdots & \vdots & \vdots & \widetilde{X} & \widetilde{P}^{+} \\
\vdots & \vdots & \vdots & \vdots & \vdots & \widetilde{P}^{-} & \widetilde{Y}
\end{array}\right), \quad \mathcal{M}=\left(\begin{array}{ccccccc}
m_{x} I & 0 & \ldots & \ldots & \ldots & \ldots & \ldots \\
0 & m_{y} I & 0 & \ldots & \ldots & \ldots & \ldots \\
\vdots & 0 & m_{u} I & 0 & \ldots & \ldots & \ldots \\
\vdots & \vdots & 0 & m_{d} I & 0 & \ldots & \ldots \\
\vdots & \vdots & \vdots & 0 & m_{s} I & 0 & \ldots \\
\vdots & \vdots & \vdots & \vdots & 0 & m_{x} I & 0 \\
\vdots & \vdots & \vdots & \vdots & \vdots & 0 & m_{y} I
\end{array}\right),
$$

where each block is a $4 \times 4$ taste matrix. The taste matrices $\xi_{T}^{(n)}$ are also enlarged to size $(N+M)^{2}$, but they maintain their flavor diagonal form, (20). In fact, for the sake of brevity we drop the superscript $(n)$ in the remaining sections. Finally, for practical calculations in S $\chi \mathrm{PT}$, it may be simplest to enlarge $\Sigma$ to be a $U(N \mid M)$ matrix, and then remove the singlet component by adding a mass term to $\mathcal{L}_{\chi}$ proportional to $m_{0}^{2} \operatorname{Str}(\Phi)^{2}$, where $m_{0}$ is sent to infinity at the end 32 .

\section{CHIRAL LAGRANGIAN FOR STAGGERED QUARKS AT NLO}

We have extended the construction reviewed in the previous two sections to NLO. At this order, the new operators introduced by discretization are proportional to $a^{2} p^{2}, a^{2} m$ and $a^{4}$. We explain in detail how we enumerate these operators in Appendix A This requires combining appropriate numbers of taste spurions, mass spurions and Lie derivatives into independent operators that are invariant under the chiral group, forming parity invariant combinations, and reducing them to a minimal set using the equations of motion. We use the graded group theory method of Ref. [36] to determine the number of linearly-independent operators. We include sources for left- and right-handed currents and scalar and pseudoscalar densities, so that we can calculate matrix elements of these operators. This leads to additional operators of $\mathcal{O}\left(a^{2} p^{2}\right)$ that are proportional to the sources, and thus contribute only to PGB decay constants. We refer to these as "source" operators. We collect all of the NLO taste-violating operators in the tables of Appendix B 
In this section we focus on the pattern of symmetry-breaking exhibited by these NLO operators. Unlike the LO staggered potential, they come from four-fermion operators in both $S_{6}^{F F(A)}$ and $S_{6}^{F F(B)}$. Thus they break the $S O(4)$ taste symmetry down to the lattice symmetry group. In order to understand the symmetries and symmetry-breaking, we examine the consequences of these operators for physical observables. We discuss the symmetries respected by these quantities order-by-order in the combined chiral-continuum expansion. It is useful to keep in mind the general form of this expansion, illustrated by the NLO PGB mass:

$$
\left(m_{\pi}^{2}\right)_{\mathrm{NLO}}=\left(m_{\pi}^{2}\right)_{\mathrm{LO}}+\left(\delta m_{\pi}^{2}\right)_{1-\text { loop }}+\left(\delta m_{\pi}^{2}\right)_{m^{2}}+\left(\delta m_{\pi}^{2}\right)_{a^{2} m}+\left(\delta m_{\pi}^{2}\right)_{a^{4}} .
$$

The subscripts indicate the order, in $\mathrm{S} \chi \mathrm{PT}$, of the terms which contribute to the physical quantity, with $m$ indicating both $m$ and $p^{2}$. We use a similar notation for the axial and pseudoscalar decay constants, $f^{A}$ and $f^{P}$. ${ }^{6}$

The LO pion mass is given in (16), while the LO decay constants are

$$
\left(f^{A}\right)_{\mathrm{LO}}=f, \quad\left(f^{P}\right)_{\mathrm{LO}}=\mu f .
$$

The results of the previous section imply that the LO contributions to these quantities respect the $S O(4)$ taste symmetry, since the potential $\mathcal{V}$ does. This has already been discussed for the PGB mass, but also holds for the decay constants. In fact, the LO decay constants are $S U(4)$ symmetric, since $\mathcal{V}$ does not contribute to either one. The staggered potential also generates interesting predictions for the difference between the properties of flavor singlet and non-singlet particles. This difference is predicted to vanish for decay constants of all tastes, and for the masses of taste $\xi_{5}$ and $\xi_{\mu \nu}$ pions (since there are only vector and axial hairpins). ${ }^{7}$ One of our aims is to determine at what order these predictions fail.

The form of the 1-loop contributions is

$$
\begin{aligned}
\left(\delta m_{\pi}^{2}\right)_{1-\text { loop }} & \sim\left[\left(m_{\pi}^{2}\right)_{\mathrm{LO}}+a^{2}\right]^{2} \ln \left(m_{\pi}^{2}\right)_{\mathrm{LO}} \\
\left(\delta f^{A, P}\right)_{1-\text { loop }} & \sim\left[\left(m_{\pi}^{2}\right)_{\mathrm{LO}}+a^{2}\right] \ln \left(m_{\pi}^{2}\right)_{\mathrm{LO}} .
\end{aligned}
$$

These involve only LO vertices, and thus do not break the $S O(4)$ taste symmetry. They do, however, break the $S U(4)$ symmetry of decay constants down to $S O(4)$. Note that, unlike in continuum $\chi \mathrm{PT}$, the coefficients of the logarithms are not proportional to the LO mass - there are additional $a^{2}$ terms from the hairpin vertices and four-pion vertices produced by $\mathcal{V}$. The exception is in the lattice Goldstone mass, which is protected by the $U(1)_{A}$ symmetry. The one-loop contributions to the lattice Goldstone mass and axial decay constant have been calculated in Ref. [13, 19].

Now we turn to the analytic NLO contributions. The generic form of these contributions are as follows:

$$
\begin{gathered}
\left(\delta m_{\pi}^{2}\right)_{m^{2}} \sim m^{2}, \quad\left(\delta m_{\pi}^{2}\right)_{a^{2} m} \sim a^{2} m, \quad\left(\delta m_{\pi}^{2}\right)_{a^{4}} \sim a^{4}, \\
\left(\delta f^{A, P}\right)_{m^{2}} \sim m, \quad\left(\delta f^{A, P}\right)_{a^{2} m} \sim a^{2} . \\
\left(\mathcal{A}^{4 \pi}\right)_{m^{2}} \sim m^{2}+m p^{2}+p^{4}, \quad\left(\mathcal{A}^{4 \pi}\right)_{a^{2} m} \sim a^{2} m+a^{2} p^{2}, \quad\left(\mathcal{A}^{4 \pi}\right)_{a^{4}} \sim a^{4} .
\end{gathered}
$$

Here we begin to include the 4-pion scattering amplitude, $A^{4 \pi}$, as it is the simplest quantity showing the most general pattern of contributions from the NLO operators. In these expressions we use a schematic notation, leaving out factors of $\Lambda_{\mathrm{QCD}}$, but distinguishing between mass and momentum-dependent terms. Note that there is no $\mathcal{O}\left(a^{4}\right)$ contribution to $f^{A, P}$ because such terms in the chiral Lagrangian contain no sources. In order to determine whether there are relationships between splittings, one needs to know which types of operators from Appendix B contribute to which of the corrections in (2729). This information is collected in Table 1 for single supertrace operators, and in Table $\llbracket$ for double supertrace operators. The former are the only contributions to the masses and decay constants of

\footnotetext{
${ }^{6}$ We use "axial decay constant" to refer to the the standard decay constant, which comes from the vacuum-to-pion matrix element of the axial current, $\left\langle 0\left|A_{\mu}\right| \pi\right\rangle \propto f^{A}$. In a slight abuse of terminology, "pseudoscalar decay constant" refers to the decay constant from the corresponding matrix element of the pseudoscalar density, $\langle 0|P| \pi\rangle \propto f^{P}$.

7 We are phrasing this discussion as if the hairpin vertices iterate to give only a mass shift for flavor singlets. As is well known, however, this is not generally true in PQ theories because valence quark loops are absent, and there are factors of $1 / 4$ added by hand due to the $\sqrt[4]{\text { Det }}$ trick. There will generally be additional, unphysical double poles in correlators 34 . Nevertheless, one can always determine the size of the hairpin vertices by measuring the residue of the double pole and comparing to the prediction of PQ S $\chi \mathrm{PT}$, as discussed below.
} 


\begin{tabular}{lccccccc}
\hline \hline \multicolumn{1}{c}{ Operator } & Table & $S O(4) ?$ & $\left(\delta m_{\pi}^{2}\right)_{a^{2} m}$ & $\left(\delta m_{\pi}^{2}\right)_{a^{4}}$ & $\left(\delta f^{A}\right)_{a^{2} m}$ & $\left(\delta f^{P}\right)_{a^{2} m}$ & $\left(\delta \mathcal{A}^{4 \pi}\right)_{a^{2} m, a^{4}}$ \\
\hline$a^{2} p^{2}(F F(A))$ & XVIIXVIII & $\mathrm{Y}$ & $\mathrm{Y}$ & $\mathrm{N}$ & $\mathrm{Y}$ & $\mathrm{Y}$ & $\mathrm{Y}$ \\
$a^{2} m(F F(A))$ & XVIIXVIII & $\mathrm{Y}$ & $\mathrm{Y}$ & $\mathrm{N}$ & $\mathrm{N}$ & $\mathrm{Y}$ & $\mathrm{Y}$ \\
$a^{4}(F F(A) \& F F(B))$ & XXXXIII & $\mathrm{Y}$ & $\mathrm{N}$ & $\mathrm{Y}$ & $\mathrm{N}$ & $\mathrm{N}$ & $\mathrm{Y}$ \\
$a^{2}$ source $(F F(A))$ & $\mathrm{XXIV}$ & $\mathrm{Y}$ & $\mathrm{N}$ & $\mathrm{N}$ & $\mathrm{Y}$ & $\mathrm{N}$ & $\mathrm{N}$ \\
\hline$a^{2} p^{2}(F F(B))$ & $\mathrm{XIX}$ & $\mathrm{N}$ & $\mathrm{Y}$ & $\mathrm{N}$ & $\mathrm{Y}$ & $\mathrm{Y}$ & $\mathrm{Y}$ \\
$a^{2}$ source $(F F(B))$ & $\mathrm{XXV}$ & $\mathrm{N}$ & $\mathrm{N}$ & $\mathrm{N}$ & $\mathrm{Y}$ & $\mathrm{N}$ & $\mathrm{N}$ \\
$a^{4}(F F(B))$ & $\mathrm{XXIII}$ & $\mathrm{N}$ & $\mathrm{N}$ & $\mathrm{N}$ & $\mathrm{N}$ & $\mathrm{N}$ & $\mathrm{Y}$ \\
\hline \hline
\end{tabular}

TABLE I: Contributions of single supertrace NLO S $\chi \mathrm{PT}$ operators to physical quantities. The column labeled "SO(4)?" indicates whether the contributions are consistent with $S O(4)$ taste symmetry. The last five columns indicate which quantities receive contributions from the particular operators. For further explanation see text.

\begin{tabular}{lcccccc}
\hline \hline \multicolumn{1}{c}{ Operator } & Table & $S O(4) ?$ & $\left(\delta m_{\pi}^{2}\right)_{a^{2} m}$ & $\left(\delta m_{\pi}^{2}\right)_{a^{4}}$ & $\left(\delta f^{A}\right)_{a^{2} m}$ & $\left(\delta f^{P}\right)_{a^{2} m}$ \\
\hline$a^{2} p^{2}(F F(A))$ & XVIIXVIII & $\mathrm{Y}$ & $\mathrm{Y}$ & $\mathrm{N}$ & $\mathrm{Y}$ & $\mathrm{Y}$ \\
$a^{2} m(F F(A))$ & XVIIXVIII & $\mathrm{Y}$ & $\xi_{\mu(5)}$ only & $\mathrm{N}$ & $\mathrm{N}$ & $\xi_{\mu(5)}$ only \\
$a^{4}(F F(A) \& F F(B))$ & XXXXIII & $\mathrm{Y}$ & $\mathrm{N}$ & $\mathrm{Y}$ & $\mathrm{N}$ & $\mathrm{N}$ \\
$a^{2}$ source $(F F(A))$ & XXIV & $\mathrm{Y}$ & $\mathrm{N}$ & $\mathrm{N}$ & $\xi_{\mu(5)}$ only & $\mathrm{N}$ \\
\hline$a^{2} p^{2}(F F(B))$ & XIX & $\mathrm{N}$ & $\mathrm{Y}$ & $\mathrm{N}$ & $\mathrm{Y}$ & $\mathrm{Y}$ \\
$a^{2}$ source $(F F(B))$ & $\mathrm{XXV}$ & $\mathrm{N}$ & $\mathrm{N}$ & $\mathrm{N}$ & $\xi_{\mu(5)}$ only & $\mathrm{N}$ \\
\hline \hline
\end{tabular}

TABLE II: Contributions of NLO S $\chi \mathrm{PT}$ operators with two supertraces to PGB masses and decay constants. Notation as in Table【 $\xi_{\mu(5)}$ indicates both vector and axial taste.

flavor non-singlet mesons, while the latter gives the additional hairpin contributions for flavor singlets. The distinction between single and double supertraces is less significant for the scattering amplitude, so we only include it in the first table.

As indicated in the tables, there is an important distinction between the contributions from underlying $F F(A)$ and $F F(B)$ four fermion operators: the former cannot break the taste $S O(4)$ symmetry, while the latter can. These two types of operator differ primarily in their index structure. Those resulting from $F F(A)$ operators must have indices contracted in pairs, while those from $F F(B)$ operators must contain more than two identical indices. This can be seen by comparing the $\mathcal{O}\left(a^{2} p^{2}\right) F F(A)$ operators in Tables XVII and XVIII to the corresponding $F F(B)$ operators in Table XIX] or the $\mathcal{O}\left(a^{4}\right) F F(A)$ operators in Tables XXXXII to the corresponding $F F(B)$ operators in Table XXIII Note, however, that the there are no $\mathcal{O}\left(a^{2} m\right)$ contributions from $F F(B)$ operators because there are not enough indices to contract more than two at a time. Thus $\mathcal{O}\left(a^{2} m\right)$ operators do not break taste $S O(4)$.

An interesting feature of the pattern of $S O(4)$ breaking is that the $\mathcal{O}\left(a^{4}\right)$ mesonic operators resulting from double insertions of underlying $F F(B)$ operators (which are listed in Table XXIII) do not break the $S O(4)$ symmetry of pion masses, despite the fact that they break $S O(4)$ in general. This is because, when calculating tree-level masses from operators such as

$$
\sum_{\mu} \sum_{\nu \neq \mu} \operatorname{Str}\left(\xi_{\mu \nu} \Sigma \xi_{\mu} \Sigma \xi_{\nu \mu} \Sigma^{\dagger} \xi_{\mu} \Sigma^{\dagger}\right)
$$

two of the four $\Sigma$ 's in each operator must be replaced by the identity, so the taste matrices collapse into a form which is $S O(4)$ symmetric. In contrast, one contribution to the four-pion scattering amplitude replaces each $\Sigma$ with a pion field, giving an $S O(4)$ breaking contribution to $\mathcal{A}^{4 \pi}$. Other contributions do not, however, break $S O(4)$. This is why, in Table【 operators of type " $a^{4} F F(B)$ " are listed as both conserving and breaking $S O(4)$, and why the latter contributes only to $\mathcal{A}^{4 \pi}$.

This distinction between two- and four-pion contributions does not arise for the $S O(4)$ breaking $\mathcal{O}\left(a^{2} p^{2}\right)$ operators. These violate $S O(4)$ by correlating indices on derivatives and taste matrices, e.g.

$$
\sum_{\mu} \operatorname{Str}\left(\Sigma D_{\mu} \Sigma^{\dagger} \xi_{\mu} \Sigma^{\dagger} D_{\mu} \Sigma \xi_{\mu}\right),
$$


where $D_{\mu}$ is the covariant derivative. They produce direction-dependent contributions to masses and decay constants, as well as to scattering amplitudes.

As is shown in the tables, the $\mathcal{O}\left(a^{2} m\right)$ operators contribute both to pion masses and to $f^{P}$ (since $M$ and $M^{\dagger}$ are sources for $P$ ), but not to $f^{A}$ (since they contain no derivatives). In particular, each $a^{2} m$ contribution to PGB properties comes with two independent mass dependencies, $m_{x}+m_{y}$ (where $x$ and $y$ are the valence flavors) and $\sum m_{\text {sea }}$, The only exception is the mass of the taste $\xi_{5}$ pion, which is guaranteed to vanish when $m_{x}+m_{y} \rightarrow 0$ by the axial symmetry, and indeed has no $a^{2} \sum m_{\text {sea }}$ contributions. Similarly, it is easy to see that it has no contributions proportional to $a^{4}$. Another feature indicated in the tables is that the " $a^{2}$ source" operators contribute only to $f{ }^{A}$.

Finally, we compare the entries in the single and double supertrace operator tables, keeping in mind that the double supertrace operators are responsible for splittings between flavor singlet and non-singlet PGB properties. The entries are almost identical. The only difference is that some of the "Y" entries in the latter are replaced by " $\xi_{\mu(5)}$ only". This is because the $a^{2} m$ and $a^{2}$ source two supertrace terms only contain vector and axial tastes. (Recall that the same is true of the LO two supertrace operators in $\mathcal{U}^{\prime}$.) In contrast, operators of $\mathcal{O}\left(a^{2} p^{2}\right)$ and $\mathcal{O}\left(a^{4}\right)$ with two supertraces contain all possible taste matrices.

We now summarize the consequences of the previous observations, with the goal in mind to generate predictions of $\mathrm{S} \chi \mathrm{PT}$ which can be tested on the lattice. We focus on the masses and decay constants, as these quantities are straightforward to calculate in simulations.

1. The $S U(4)$ symmetry in the LO decay constants of flavor non-singlet PGBs is broken down to $S O(4)$ by the NLO terms resulting from $F F(A)$ operators. ${ }^{8}$ There is no relation between the $S U(4) \rightarrow S O(4)$ breaking in $f^{A}$ and $f^{P}$, since the $a^{2} p^{2}$ and $a^{2} m$ operators contribute differently to these two decay constants. Furthermore, 1-loop contributions to $m_{\pi}^{2}, f^{A}$ and $f^{P}$ will give independent contributions to the splittings. Thus there are no predictions between splittings at this stage.

2. The breaking of $S O(4)$ down to $\Gamma_{4} \rtimes S W_{4}$ occurs first in the NLO analytic terms, and not in the 1-loop terms. It arises only from two types of operator $-a^{2} p^{2}$ and " $a^{2}$ source". In particular, since both $m_{\pi}$ and $f^{P}$ only receive $S O(4)$-breaking contributions from $\mathcal{O}\left(a^{2} p^{2}\right)$ operators, there are relations between the splittings within $S O(4)$ multiplets. The " $a^{2}$ source" operators break all such relations among splittings in $f^{A}$. There are also predictions for the rotational symmetry breaking in the dispersion relations. These results hold separately for flavor singlet and non-singlet pions.

3. The absence of taste $\xi_{5}$ and $\xi_{\mu \nu}$ hairpins at LO does not hold at NLO, where there are hairpin vertices for all tastes. We note that the presence of a hairpin vertex for taste $\xi_{5}$ is consistent with its Goldstone nature since the vertex is proportional to $p^{2}$, and thus $m_{\pi}^{2}$ at the pole.

4. The hairpin contributions to $f^{A}$ and $f^{P}$ are related to each other for tastes $\mathrm{P}$ and $\mathrm{T}$, since there are no two supertrace operators with sources for either the pseudoscalar density or the axial current. This is true for both the $S O(4)$-conserving and the $S O(4)$-violating contributions.

We discuss the various relations and predictions in detail in the following section. Here we stress that none of these relations follows from a symmetry of the lattice theory - indeed, we have checked that all of them are broken by higher dimension operators in $\mathrm{S} \chi \mathrm{PT}$.

\section{NLO RELATIONS FOR PGB MASSES AND DECAY CONSTANTS}

In this section we work out the detailed form of the relations which follow from the particular form of the NLO analytic terms.

We first study $S O(4)$-taste and rotational symmetry breaking in the pion dispersion relations. This arises from the $a^{2} p^{2} F F(B)$ operators, which are listed in Table XIX Of the 18 such operators, only 8 contribute to $S O(4)$ breaking in single pion properties. The others, such as

$$
\sum_{\mu} \sum_{\nu \neq \mu} \operatorname{Str}\left(\partial_{\mu} \Sigma^{\dagger} \partial_{\mu} \Sigma \Sigma^{\dagger} \xi_{\mu \nu} \Sigma \xi_{\nu \mu}\right)+\text { p.c. }
$$

\footnotetext{
8 We have checked that there are sufficient independent operators to completely break the symmetry down to $S U(4)$ in this and all other similar cases that we mention.
} 
(with p.c. indicating parity conjugate) give either vanishing, or taste symmetric, contributions to two pion properties, because the two pions must be drawn from the fields with derivatives acting on them. The contribution of the $S O(4)$ breaking operators to the chiral Lagrangian is

$$
\begin{aligned}
& a^{2} \sum_{\mu} \sum_{\nu \neq \mu}\left\{C_{2} \operatorname{Str}\left(\partial_{\mu} \Sigma^{\dagger} \xi_{\mu \nu} \partial_{\mu} \Sigma \xi_{\nu \mu}\right)+C_{7} \operatorname{Str}\left(\Sigma \partial_{\mu} \Sigma^{\dagger} \xi_{\mu \nu}\right) \operatorname{Str}\left(\Sigma^{\dagger} \partial_{\mu} \Sigma \xi_{\nu \mu}\right)\right. \\
& \left.+C_{10}\left[\operatorname{Str}\left(\Sigma \partial_{\mu} \Sigma^{\dagger} \xi_{\mu \nu} \Sigma \partial_{\mu} \Sigma^{\dagger} \xi_{\nu \mu}\right)+p . c .\right]+C_{13}\left[\operatorname{Str}\left(\Sigma \partial_{\mu} \Sigma^{\dagger} \xi_{\mu \nu}\right) \operatorname{Str}\left(\Sigma \partial_{\mu} \Sigma^{\dagger} \xi_{\nu \mu}\right)+p . c .\right]\right\} \\
& +a^{2} \sum_{\mu}\left\{C_{36 V} \operatorname{Str}\left(\Sigma \partial_{\mu} \Sigma^{\dagger} \xi_{\mu} \Sigma^{\dagger} \partial_{\mu} \Sigma \xi_{\mu}\right)+C_{36 A} \operatorname{Str}\left(\Sigma \partial_{\mu} \Sigma^{\dagger} \xi_{\mu 5} \Sigma^{\dagger} \partial_{\mu} \Sigma \xi_{5 \mu}\right)\right. \\
& \left.+C_{41 V} \operatorname{Str}\left(\partial_{\mu} \Sigma^{\dagger} \xi_{\mu}\right) \operatorname{Str}\left(\partial_{\mu} \Sigma \xi_{\mu}\right)+C_{41 A} \operatorname{Str}\left(\partial_{\mu} \Sigma^{\dagger} \xi_{\mu 5}\right) \operatorname{Str}\left(\partial_{\mu} \Sigma \xi_{5 \mu}\right)\right\} .
\end{aligned}
$$

The eight operators reduce to six for two pion contributions, three single supertrace and three double supertrace operators:

$$
\begin{aligned}
& \frac{a^{2}}{f^{2}} \sum_{\mu}\left\{\sum_{\nu \neq \mu}\left[\left(C_{2}-2 C_{10}\right) \operatorname{Str}\left(\partial_{\mu} \Phi \xi_{\mu \nu} \partial_{\mu} \Phi \xi_{\nu \mu}\right)+\left(C_{7}-2 C_{13}\right) \operatorname{Str}\left(\partial_{\mu} \Phi \xi_{\mu \nu}\right) \operatorname{Str}\left(\partial_{\mu} \Phi \xi_{\mu \nu}\right)\right]\right. \\
& \quad+C_{36 V} \operatorname{Str}\left(\partial_{\mu} \Phi \xi_{\mu} \partial_{\mu} \Phi \xi_{\mu}\right)+C_{41 V} \operatorname{Str}\left(\partial_{\mu} \Phi \xi_{\mu}\right) \operatorname{Str}\left(\partial_{\mu} \Phi \xi_{\mu}\right) \\
& \left.\quad+C_{36 A} \operatorname{Str}\left(\partial_{\mu} \Phi \xi_{\mu 5} \partial_{\mu} \Phi \xi_{5 \mu}\right)+C_{41 A} \operatorname{Str}\left(\partial_{\mu} \Phi \xi_{\mu 5}\right) \operatorname{Str}\left(\partial_{\mu} \Phi \xi_{5 \mu}\right)\right\} .
\end{aligned}
$$

We consider first the effect of these operators on the properties of flavor non-singlet mesons, and in particular those composed of a quark of flavor $a$ and an antiquark of a different flavor $b$, where these flavors can be either valence or sea quarks. Examples are kaons and charged pions, both unquenched and partially quenched. ${ }^{9}$ For these particles only the single supertrace operators contribute, and we find the following results for their dispersion relations:

$$
\begin{aligned}
E_{I}^{2} & =\vec{p}^{2}+m_{I}^{2}\left(1+\delta_{I}\right) \\
E_{5}^{2} & =\vec{p}^{2}+m_{5}^{2}\left(1+\delta_{5}\right) \\
E_{k}^{2} & =\left(p_{i}^{2}+p_{j}^{2}\right)+p_{k}^{2}\left(1+\delta_{k}-\delta_{4}\right)+m_{\mu}^{2}\left(1+\delta_{k}\right) \\
E_{4}^{2} & =\vec{p}^{2}\left(1+\delta_{4}-\delta_{k}\right)+m_{\mu}^{2}\left(1+\delta_{4}\right) \\
E_{k 5}^{2} & =\left(p_{i}^{2}+p_{j}^{2}\right)+p_{k}^{2}\left(1+\delta_{k 5}-\delta_{45}\right)+m_{\mu 5}^{2}\left(1+\delta_{k 5}\right) \\
E_{45}^{2} & =\vec{p}^{2}\left(1+\delta_{45}-\delta_{k 5}\right)+m_{\mu 5}^{2}\left(1+\delta_{45}\right) \\
E_{l m}^{2} & =\left(p_{l}^{2}+p_{m}^{2}\right)\left(1+\delta_{l m}-\delta_{k 4}\right)+p_{k}^{2}+m_{\mu \nu}^{2}\left(1+\delta_{l m}\right) \\
E_{k 4}^{2} & =\left(p_{l}^{2}+p_{m}^{2}\right)\left(1+\delta_{k 4}-\delta_{l m}\right)+p_{k}^{2}+m_{\mu \nu}^{2}\left(1+\delta_{k 4}\right) .
\end{aligned}
$$

Here $E_{F}$ is the energy of the taste $F$ PGB determined from the exponential fall-off of the two-point function along the Euclidean time direction. The quantities $m_{F}$ are the masses including all NLO contributions except that from the $S O(4)$ breaking operators in (34). They are thus labeled by indices of the $S O(4)$ irreps: $I, 5, \mu, \mu 5$ and $\mu \nu$. The additional contributions from the $S O(4)$ breaking operators are denoted $\delta_{F}$. Here the labels, like those on the energies, are those of the 8 irreps of the lattice timeslice group, which distinguishes between spatial and timelike indices [37]. This is the maximal splitting among tastes consistent with the lattice symmetry for states at zero spatial momentum. The expressions show that, at non-zero momentum, the states fall into even smaller representations, due to the coupling of the spin and taste transformations in the lattice symmetry group. These irreps have been discussed in Ref. [30].

The expressions for the $S O(4)$ breaking mass shifts $\left(\delta m_{\pi}^{2} / m_{\pi}^{2}\right)$ are

$$
\begin{aligned}
& \delta_{I}=-\frac{8 a^{2}}{f^{2}}\left(C_{36 V}+C_{36 A}\right), \\
& \delta_{5}=\frac{8 a^{2}}{f^{2}}\left(C_{36 V}+C_{36 A}\right),
\end{aligned}
$$

\footnotetext{
${ }^{9}$ The following relations also hold for flavor neutral mesons, e.g the $\pi_{0}$-like states with flavor structure $(a a-b b)$, if flavors $a$ and $b$ are degenerate.
} 


$$
\begin{aligned}
\delta_{k} & =-\frac{8 a^{2}}{f^{2}}\left(C_{2}-2 C_{10}\right)+\frac{8 a^{2}}{f^{2}}\left(C_{36 V}-C_{36 A}\right), \\
\delta_{4} & =+\frac{24 a^{2}}{f^{2}}\left(C_{2}-2 C_{10}\right)-\frac{8 a^{2}}{f^{2}}\left(C_{36 V}-C_{36 A}\right), \\
\delta_{k 5} & =-\frac{8 a^{2}}{f^{2}}\left(C_{2}-2 C_{10}\right)-\frac{8 a^{2}}{f^{2}}\left(C_{36 V}-C_{36 A}\right), \\
\delta_{45} & =+\frac{24 a^{2}}{f^{2}}\left(C_{2}-2 C_{10}\right)+\frac{8 a^{2}}{f^{2}}\left(C_{36 V}-C_{36 A}\right), \\
\delta_{l m} & =-\frac{8 a^{2}}{f^{2}}\left(C_{36 V}+C_{36 A}\right), \\
\delta_{k 4} & =+\frac{8 a^{2}}{f^{2}}\left(C_{36 V}+C_{36 A}\right) .
\end{aligned}
$$

There are three $S O(4)$ breaking splittings, those between tastes $\xi_{4}$ and $\xi_{i}$, between $\xi_{45}$ and $\xi_{i 5}$, and between $\xi_{i j}$ and $\xi_{k 4}$. The six independent coefficients are sufficient to give independent contributions to each of these splittings. Thus there are no relations predicted between these mass splittings.

What is predicted, however, are relations between the violation of rotation symmetry in the dispersion relations and the $S O(4)$-taste breaking in the mass splittings. These can be written as

$$
\begin{aligned}
\frac{E_{k}^{2}-E_{4}^{2}}{m_{k}^{2}-m_{4}^{2}} & =1+\frac{p_{i}^{2}+p_{j}^{2}+2 p_{k}^{2}}{\left(m_{k}^{2}+m_{4}^{2}\right) / 2}, \\
\frac{E_{k 5}^{2}-E_{45}^{2}}{m_{k 5}^{2}-m_{45}^{2}} & =1+\frac{p_{i}^{2}+p_{j}^{2}+2 p_{k}^{2}}{\left(m_{k 5}^{2}+m_{45}^{2}\right) / 2}, \\
\frac{E_{l m}^{2}-E_{k 4}^{2}}{m_{l m}^{2}-m_{k 4}^{2}} & =1+2 \frac{p_{l}^{2}+p_{m}^{2}}{\left(m_{l m}^{2}+m_{k 4}^{2}\right) / 2} .
\end{aligned}
$$

In these relations $m_{F}$ is the full NLO mass. Note that the relations are trivial when $\vec{p}=0$. The fact that the precise form of the mass used in the denominator on the r.h.s. does not matter at this order allows these expressions to be written in terms of quantitities that are directly measurable on the lattice.

Note that neither the taste singlet nor the taste Goldstone PGBs receive rotational symmetry violating contributions to their dispersion relations at this order. Such terms are prohibited by the fact that these tastes transform trivially under rotations. Therefore they can only feel the effect of rotational symmetry breaking through operators with four or more derivatives [13],

$$
a^{2} \sum_{\mu} \partial_{\mu}^{2} \pi \partial_{\mu}^{2} \pi
$$

which are of $\mathcal{O}\left(a^{2} p^{4}\right)$ and thus contribute only at NNLO.

Next we turn to the relations involving the decay constants, still considering only flavor non-singlet mesons. As explained in the previous section, the $S O(4)$ breaking operators of $\mathcal{O}\left(a^{2} p^{2}\right)$ contribute to both the masses and the decay constants. For the pseudoscalar decay constants, as for the masses, the contribution is through wavefunction renormalization. Since the same terms contribute to both masses and decay constants, there are three simple relationships between splittings:

$$
\begin{aligned}
\left(\frac{f_{k}^{P}-f_{4}^{P}}{f_{k}^{P}+f_{4}^{P}}\right) & =\frac{1}{2}\left(\frac{m_{k}^{2}-m_{4}^{2}}{m_{k}^{2}+m_{4}^{2}}\right), \\
\left(\frac{f_{k 5}^{P}-f_{45}^{P}}{f_{k 5}^{P}+f_{45}^{P}}\right) & =\frac{1}{2}\left(\frac{m_{k 5}^{2}-m_{45}^{2}}{m_{k 5}^{2}+m_{45}^{2}}\right), \\
\left(\frac{f_{l m}^{P}-f_{k 4}^{P}}{f_{l m}^{P}+f_{k 4}^{P}}\right) & =\frac{1}{2}\left(\frac{m_{l m}^{2}-m_{k 4}^{2}}{m_{l m}^{2}+m_{k 4}^{2}}\right) .
\end{aligned}
$$

These are likely to be the most simple predictions to test in practice. It is essential for these expressions that the $Z$-factors are $S O(4)$-invariant, and therefore identical for both tastes in the expression. They can thus be tested using bare lattice operators, thereby avoiding $\mathcal{O}\left(a^{2}\right)$ ambiguities in matching lattice and continuum operators that could 
destroy the relationship. This is not true in general (e.g. for $f^{A}$ ) but does hold for the pseudoscalar operators $\gamma_{5} \otimes \xi_{F}$. For example, the operators with spin-taste structure $\gamma_{5} \otimes \xi_{i}$ are related to $\gamma_{5} \otimes \xi_{4}$ by a lattice rotation.

This argument shows why there can be no similar relationships involving the axial decay constants, since the $Z$ factors in that case are not $S O(4)$ invariant. For example, $\gamma_{45} \otimes \xi_{i}$ is a "2 link" operator while $\gamma_{45} \otimes \xi_{4}$ is a "4 link" operator, so they are clearly not related by any lattice symmetries, and thus have different $Z$ 's. In fact, it is not even clear how to unambiguously calculate the quantities on the l.h.s. of eq. (46) for $f^{P} \rightarrow f^{A}$, as the $Z$-factors are not

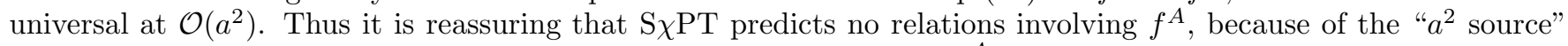
mesonic operators, which contribute $S O(4)$ breaking contributions to $f^{A}$ alone. The only prediction for axial decay constants is a qualitative one: the splittings between $S O(4)$ irreps should not be different in magnitude from those within the irreps. In other words, there should be no pattern of approximate degeneracies, unlike for the masses which exhibit approximate $S O(4)$ symmetry. This expectation is consistent with the results of Ref. [16], in which $f^{A}$ was calculated for all tastes in the quenched approximation.

We now turn to $S O(4)$-breaking predictions for PGBs for which the double supertrace, or "hairpin", operators also contribute. These are particles which have a flavor singlet component, i.e. $\bar{u} u+\bar{d} d$ and $\bar{s} s$ in the unquenched sector, and $\bar{x} x$ or $\bar{y} y$ (and their ghost companions) in the valence sector. These states are mixed by hairpin contributions, and because, in general, they start off before mixing with different masses (due to the different masses of the quarks), there are no simple predictions for the properties of the resulting mixed states. The situation is yet more complicated if the $\sqrt[4]{\text { Det }}$ trick is being used. In fact, for the vector and axial tastes these complications arise at LO, due to the presence of hairpins, and have been addressed in detail in Ref. [13].

The only theories for which the flavor non-singlet predictions still hold as written are unquenched theories with $N \geq 1$ degenerate flavors. In these theories there is a single flavor-singlet state, and thus no mixing. This state can have any taste except $\xi_{I}$, which is, as noted above, not a PGB. The predictions for the other fifteen tastes take exactly the same form as those given above for the flavor non-singlets, except that there are additional contributions to the $\delta$ 's from the double supertrace operators:

$$
\begin{aligned}
\delta_{4}^{\text {singlet }} & =-N \frac{32 a^{2}}{f^{2}} C_{41 V}, \\
\delta_{45}^{\text {singlet }} & =-N \frac{32 a^{2}}{f^{2}} C_{41 A}, \\
\delta_{k 4}^{\text {singlet }} & =-N \frac{16 a^{2}}{f^{2}}\left(C_{7}-2 C_{13}\right) .
\end{aligned}
$$

Thus the $S O(4)$ breaking splittings for the flavor singlets are not related to those of the non-singlets, but the form of the predictions for dispersion relations, (44), and of the relations between mass and decay constant splittings, (46), remain unchanged.

The situation is similar if one has $N$ degenerate flavors and uses the $\sqrt[4]{\text { Det }}$ trick. The only complication is that the disconnected quark contractions must be multiplied, by hand, by a factor of $1 / 4$ before being added to the connected quark contractions. Then the predictions of (44) and (46) apply, with the $\delta$ 's having the additional contributions given in (47), except that $N \rightarrow N / 4$.

For theories with non-degenerate sea quarks, such as QCD, and for PQ theories, there are no simple relations involving masses and decay constants. Instead, we can obtain simple relations involving the hairpin vertices by considering disconnected valence correlators. These have the flavor structure $11 \leftrightarrow 22$, where 1 and 2 refer to valence flavors $(x, y, \ldots)$. This picks out the desired hairpin contractions at the quark level. To cancel the LO decay constants, and to obtain a quantity with a simple dependence on Euclidean time, we take the ratio of the disconnected correlator to the connected $(12 \leftrightarrow 21)$ correlator. This ratio has been studied extensively in the quenched approximation. In the present context, it has the attractive property that the derived relationships hold even if one uses the $\sqrt[4]{\text { Det }}$ trick. For simplicity, we restrict ourselves to degenerate valence quarks, i.e. $m_{1}=m_{2}$.

The earlier flavor nonsinglet predictions related splittings within $S O(4)$ irreps in masses and pseudoscalar decay constants. As a natural generalization to flavor singlets, we therefore consider $S O(4)$-breaking in disconnected correlators of the pseudoscalar density. For example, for tensor taste we calculate

$$
\frac{\left\langle P_{11}(0) P_{22}(\vec{p}=0, t)\right\rangle_{\xi_{\ell m}}-\left\langle P_{11}(0) P_{22}(\vec{p}=0, t)\right\rangle_{\xi_{k 4}}}{\left\langle P_{11}(0) P_{22}(\vec{p}=0, t)\right\rangle_{\xi_{\mu \nu}}},
$$

where $P$ indicates a pseudoscalar operator with the flavor indicated by the subscript. The taste of the operators are shown by the subscript to the expectation value. Note that, in the denominator, any choice of tensor taste can be used, as the difference between the correlators for timeslice irreps $\xi_{\ell m}$ and $\xi_{k 4}$ is subleading. 


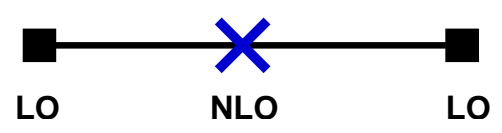

FIG. 1: NLO contribution to the $\langle P P\rangle$ flavor-disconnected correlator. The two black squares represent insertions of the pseudoscalar density, while the cross represents the hairpin vertex. For tensor taste, this vertex comes from both $\mathcal{O}\left(a^{2} p^{2}\right)$ and $\mathcal{O}\left(a^{4}\right)$ two supertrace operators, but only two of the $\mathcal{O}\left(a^{2} p^{2}\right)$ operators lead to $S O(4)$ breaking. The analogous diagram, with pseudoscalar sources changed to axial currents, contributes to the $\langle A A\rangle$ flavor-disconnected correlator.

We describe the calculation of (48) in detail, as the remaining predictions in this section all rely on some generalization of this method. At NLO, the numerator is due to one diagram, shown in Figure 11 in which two LO insertions of the flavor singlet pseudoscalar density are contracted with a NLO hairpin vertex. While the full NLO tensor taste hairpin vertex comes from both $\mathcal{O}\left(a^{2} p^{2}\right)$ and $\mathcal{O}\left(a^{4}\right)$ operators, only two operators, $C_{7}$ and $C_{13}$ in (33), produce $S O(4)$-breaking. ${ }^{10}$ Thus, before Fourier transformation, the numerator of (48) is

$$
\begin{aligned}
\left\langle P_{11}(0) P_{22}(p)\right\rangle_{\xi_{\ell m}}-\left\langle P_{11}(0) P_{22}(p)\right\rangle_{\xi_{k 4}} & =16 a^{2} \mu^{2} p^{2}\left(C_{7}-2 C_{13}\right)\left(\frac{1}{p^{2}+m_{\mu \nu}^{2}}\right)^{2} \\
& =16 a^{2} \mu^{2}\left(C_{7}-2 C_{13}\right)\left\{\frac{1}{p^{2}+m_{\mu \nu}^{2}}-m_{\mu \nu}^{2}\left(\frac{1}{p^{2}+m_{\mu \nu}^{2}}\right)^{2}\right\} .
\end{aligned}
$$

The mass $m_{\mu \nu}$ is that of the non-singlet taste tensor meson with flavor 12 . In this equation it is the LO mass, which is the same for all components of the $S O(4)$ irrep. At NLO accuracy, it can, however, be replaced by the NLO (or all orders) mass of any member of the irrep. In the second line we have shown explicitly the single and double-pole contributions. In an unquenched theory, the former would give rise to a correction to the pseudoscalar decay constant, the latter to the PGB mass. The $\mathrm{S} \chi \mathrm{PT}$ prediction is that they have a common coefficient since they result from the same operators.

To denominator of the ratio (48) is just the LO contribution to the connected $\langle P P\rangle$ correlator. Before Fourier transformation it is

$$
\left\langle P_{11}(0) P_{22}(p)\right\rangle_{\xi_{\mu \nu}}=\mu^{2} f^{2} \frac{1}{p^{2}+m_{\mu \nu}^{2}} .
$$

We now set $\vec{p}=0$, Fourier transform to Euclidean time, and form the ratio, yielding:

$$
\begin{aligned}
\frac{\left\langle P_{11}(0) P_{22}(\vec{p}=0, t)\right\rangle_{\xi_{\ell m}}-\left\langle P_{11}(0) P_{22}(\vec{p}=0, t)\right\rangle_{\xi_{k 4}}}{\left\langle P_{11}(0) P_{22}(\vec{p}=0, t)\right\rangle_{\xi_{\mu \nu}}} & =-\delta_{k 4}^{\operatorname{singlet}}\left(1-\left[1+m_{\mu \nu}|t|\right] / 2\right) \\
& =-\delta_{k 4}^{\operatorname{singlet}}\left(1-m_{\mu \nu}|t|\right) / 2
\end{aligned}
$$

where $t$ is Euclidean time, and $\delta_{k 4}^{\text {singlet }}$ is given in (47) with $N=1$. On the first line, the 1 on the r.h.s. is from the single pole in the numerator, while the $\left[1+m_{\mu \nu}|t|\right] / 2$ is from the double pole. We imagine that the mass $m_{\mu \nu}$ is first determined from the connected correlator. The prediction of $\mathrm{S} \chi \mathrm{PT}$ is then that the constant term and the term linear in $m_{\mu \nu}|t|$ should have opposite coefficients. That it is possible to separate these two terms has been shown in quenched simulations [35]. Note that for this prediction to hold one must be at large enough Euclidean times that the PGB contributions dominate the correlators in both the numerator and denominator of the ratio, but small enough that $\left|\delta_{k 4}^{\text {singlet }} m_{\mu \nu} t\right| \ll 1$. If the latter condition does not hold, further terms in the iteration of the NLO hairpin must be included.

As noted above, the prediction (52) holds irrespective of whether the $\sqrt[4]{\text { Det }}$ trick has been used. This is because the hairpin correlators do not, at this order, access the sea sector of the theory. The only caveat is that one must ensure that the mass of the tensor taste valence mesons differs significantly from that of any of the tensor taste mesons composed of sea quarks. If not, mixing of valence and sea sectors, although of higher than NLO, can be enhanced by the proximity to intermediate poles.

Another feature of the flavor-singlet prediction common with those earlier is that one can use the bare lattice pseudoscalar densities, since $Z$ factors cancel in the ratio. One might be concerned that the $Z$ factors in the numerator

10 Recall that $\mathcal{O}\left(a^{4}\right)$ operators are $S O(4)$-invariant for 2-PGB properties, and only two-supertrace operators produce hairpin vertices. 
and denominator might differ, since the calculation of the former involves additional quark disconnected diagrams with intermediate gluons. One can show, however, that the difference between the $Z$ factors vanishes in the continuum limit. This is actually true irrespective of the spin of the bilinear and follows because the operator has non-trivial taste. Thus the difference between $Z$ 's in the numerator and denominator is of $O\left(a^{2}\right)$, and does not contribute at NLO.

We have not been able to find a prediction as simple as (52) for the vector or axial taste PGBs. The analogous $S O(4)$ breaking quantity to consider for, say, vector taste is:

$$
\frac{\left\langle P_{11}(0) P_{22}(\vec{p}=0, t)\right\rangle_{\xi_{4}}-\left\langle P_{11}(0) P_{22}(\vec{p}=0, t)\right\rangle_{\xi_{k}}}{\left\langle P_{11}(0) P_{22}(\vec{p}=0, t)\right\rangle_{\xi_{\mu}}}
$$

Using the methods of Refs. 13, 32] one can determine the form of this ratio. The calculation is complicated by the presence of the LO hairpin vertex, which means that there are not only terms proportional to $\delta_{4}^{\text {singlet }}$, but also terms proportional to the non-singlet $S O(4)$ breaking quantity $\left(\delta_{4}-\delta_{k}\right)$. Furthermore, the fact that the LO hairpins must be iterated to all orders brings in the sea sector through intermediate propagators, and the momentum dependence is quite complicated. Thus we do not give explicit expressions.

Finally, we discuss the fourth point of our summary in the previous section. We can relate the hairpin contributions to $f^{P}$ and $f^{A}$ for pseudoscalar and tensor taste because they only arise from operators of $\mathcal{O}\left(a^{2} p^{2}\right)$ and $\mathcal{O}\left(a^{4}\right)$. In particular, there are no two supertrace $\mathcal{O}\left(a^{2} m\right)$ operators, which would contribute to $f^{P}$, or $\mathcal{O}\left(a^{2}\right.$ source $)$ operators, which would only modify $f^{A}$; all NLO hairpin operators contribute to either both or neither quantity. It is a straightforward exercise to calculate the contribution of these operators to $f^{P}$ and $f^{A}$, and, when we do, we find a simple relation for both tastes:

$$
\left(\frac{\delta f_{5}^{P}}{f^{P}}\right)_{\text {hairpin }}=-\left(\frac{\delta f_{5}^{A}}{f^{A}}\right)_{\text {hairpin }}, \quad\left(\frac{\delta f_{\mu \nu}^{P}}{f^{P}}\right)_{\text {hairpin }}=-\left(\frac{\delta f_{\mu \nu}^{A}}{f^{A}}\right)_{\text {hairpin }} .
$$

The contributions of the NLO two supertrace operators are equal and opposite. The minus sign comes from the fact that the $\mathcal{O}\left(a^{2} p^{2}\right)$ terms contribute to $f^{P}$ only through wavefunction renormalization, but to $f^{A}$ also through the axial sources contained in the covariant derivative. Note that these relationships do involve both $S O(4)$ conserving and breaking NLO contributions - in this sense they are more general than those considered above.

These expressions can only be evaluated as written in unquenched theories with degenerate quarks. One needs to determine the decay constants of flavor singlet and non-singlet PGBs, and take the difference-this is $\delta f_{\text {hairpin. For }}$ non-degenerate sea quarks and/or PQ theories, however, this will not work. As above, one can instead isolate the hairpin contributions using disconnected valence correlation functions. We consider the following ratios of pseudoscalar densities and axial currents:

$$
\frac{\left\langle P_{11}(0) P_{22}(\vec{p}=0, t)\right\rangle_{\xi_{T}}}{\left\langle P_{12}(0) P_{21}(\vec{p}=0, t)\right\rangle_{\xi_{T}}}, \quad \frac{\left\langle A_{11}^{4}(0) A_{22}^{4}(\vec{p}=0, t)\right\rangle_{\xi_{T}}}{\left\langle A_{12}^{4}(0) A_{21}^{4}(\vec{p}=0, t)\right\rangle_{\xi_{T}}} .
$$

The superscript on the axial currents indicates the Lorentz index, and not taste. Again we assume equal valence quark masses, $m_{1}=m_{2}$, and that the valence PGBs have masses differing from those of all PGBs composed of sea quarks. In both these ratios bare operators can be used as $Z$ factors cancel as above.

First consider taste $\xi_{5}$. In this case $\mathcal{O}\left(a^{4}\right)$ hairpin vertices are prohibited because of the lattice axial symmetry, so that the numerators of the ratios only involve two operators:

$$
a^{2} \sum_{\mu}\left\{C_{7 P} \operatorname{Str}\left(\Sigma \partial_{\mu} \Sigma^{\dagger} \xi_{5}\right) \operatorname{Str}\left(\Sigma^{\dagger} \partial_{\mu} \Sigma \xi_{5}\right)+C_{13 P}\left[\operatorname{Str}\left(\Sigma \partial_{\mu} \Sigma^{\dagger} \xi_{5}\right) \operatorname{Str}\left(\Sigma \partial_{\mu} \Sigma^{\dagger} \xi_{5}\right)+p . c .\right]\right\}
$$

The contribution to the pseudoscalar ratio can be worked out as above. Only the diagram of Fig. 1 contributes to the numerator, and one finds at NLO

$$
\frac{\left\langle P_{11}(0) P_{22}(\vec{p}=0, t)\right\rangle_{\xi_{5}}}{\left\langle P_{12}(0) P_{21}(\vec{p}=0, t)\right\rangle_{\xi_{5}}}=-\frac{16 a^{2} \mu^{2}}{f^{2}}\left(C_{7 P}-2 C_{13 P}\right)\left(\frac{1-m_{5}|t|}{2}\right) .
$$

In fact, without even relating this to the $\langle A A\rangle$ ratio, we have another prediction of $\mathrm{S} \chi \mathrm{PT}$ : the constant and $m_{5}|t|$ terms should have opposite signs.

For the axial current correlator, the numerator receives contributions not only from wave function renormalization (Fig. 1), but also through direct renormalization of the axial current (shown in Fig. 2). The flavor-disconnected axial 


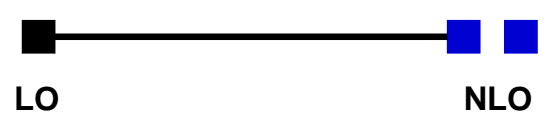

FIG. 2: NLO contribution to the $\langle A A\rangle$ flavor-disconnected correlator from axial current renormalization. The single black square represents an insertion of the LO axial current, while the double box represents an insertion of the NLO axial current. It is shown as two boxes because the hairpin vertex is in the current itself.

correlator for taste $\xi_{5}$ is:

$$
\left\langle A_{11}^{4}(0) A_{22}^{4}(p)\right\rangle_{\xi_{5}}=-16 a^{2}\left(C_{7 P}-2 C_{13 P}\right) p_{4}^{2}\left\{\frac{p^{2}}{\left(p^{2}+m_{5}^{2}\right)^{2}}-2 \frac{1}{\left(p^{2}+m_{5}^{2}\right)}\right\},
$$

where the first term is from wavefunction renormalization, and the second is from axial current renormalization. If we set $\vec{p}=0$, this can be simplified to:

$$
\left\langle A_{11}^{4}(0) A_{22}^{4}\left(\vec{p}=0, p_{4}\right)\right\rangle_{\xi_{5}}=16 a^{2}\left(C_{7 P}-2 C_{13 P}\right)\left\{2-\frac{m_{5}^{4}}{\left(p_{4}^{2}+m_{5}^{2}\right)^{2}}\right\} .
$$

Thus, in the axial correlator, the fact that only $\mathcal{O}\left(a^{2} p^{2}\right)$ operators contribute for taste $\xi_{5}$ results in the absence of a single pole term. To test this in practice one Fourier transforms and takes the ratio to the connected correlator:

$$
\frac{\left\langle A_{11}^{4}(0) A_{22}^{4}(\vec{p}=0, t)\right\rangle_{\xi_{5}}}{\left\langle A_{12}^{4}(0) A_{21}^{4}(\vec{p}=0, t)\right\rangle_{\xi_{5}}}=-\frac{16 a^{2} \mu^{2}}{f^{2}}\left(C_{7 P}-2 C_{13 P}\right)\left(\frac{-1-m_{5}|t|}{2}\right) .
$$

The absence of a single pole translates into the predicted $\left(1+m_{5}|t|\right)$ dependence.

Now we can return to the relation between hairpin contributions to $f^{P}$ and $f^{A}$. This shows up in the fact that the overall coefficients in the $\langle P P\rangle$ and $\langle A A\rangle$ ratios are the same. The prediction in (54) becomes the result that the constant terms have opposite signs. On the other hand, the linear terms must have the same sign as they correpond to a mass shift which does not depend on the external operators. One way to test the prediction of opposite constant terms is to add the two ratios:

$$
\frac{\left\langle P_{11}(0) P_{22}(\vec{p}=0, t)\right\rangle_{\xi_{5}}}{\left\langle P_{12}(0) P_{21}(\vec{p}=0, t)\right\rangle_{\xi_{5}}}+\frac{\left\langle A_{11}^{4}(0) A_{22}^{4}(\vec{p}=0, t)\right\rangle_{\xi_{5}}}{\left\langle A_{12}^{4}(0) A_{21}^{4}(\vec{p}=0, t)\right\rangle_{\xi_{5}}} \propto m_{5}|t|
$$

The $\mathrm{S} \chi \mathrm{PT}$ prediction at NLO is then that there is no constant term in this quantity.

Because the tensor taste PGB is not a lattice Goldstone pion, it has $\mathcal{O}\left(a^{4}\right)$ hairpin vertices in addition to those of $\mathcal{O}\left(a^{2} p^{2}\right)$. Because of this, neither of the predictions (57) or (60) apply - the constant and linear terms in $|t|$ within each individual ratio are not related. Since the $\mathcal{O}\left(a^{4}\right)$ hairpins lead only to mass renormalization, one expects, however, for the constant terms in the $\langle P P\rangle$ and $\langle A A\rangle$ ratios to be opposite, as for taste $\xi_{5}$. This turns out to be the case. Thus we find

$$
\frac{\left\langle P_{11}(0) P_{22}(\vec{p}=0, t)\right\rangle_{\xi_{\mu \nu}}}{\left\langle P_{12}(0) P_{21}(\vec{p}=0, t)\right\rangle_{\xi_{\mu \nu}}}+\frac{\left\langle A_{11}^{4}(0) A_{22}^{4}(\vec{p}=0, t)\right\rangle_{\xi_{\mu \nu}}}{\left\langle A_{12}^{4}(0) A_{21}^{4}(\vec{p}=0, t)\right\rangle_{\xi_{\mu \nu}}} \propto m_{\mu \nu}|t|
$$

The absence of a constant term in this quantity is really two independent predictions, one for taste $\xi_{4 k}$ and another for $\xi_{\ell m}$.

Since the predictions (57), (60) and (61) do not require $S O(4)$ breaking, there can be and in fact are one-loop contributions to these ratios involving LO vertices. Note that, as discussed in Ref. [32], quark disconnected correlators receive contributions at one-loop from single supertrace vertices. We have evaluated this contributions, and find that they are consistent with all three predictions. Thus these are full NLO predictions of S $\chi \mathrm{PT}$.

\section{CONCLUSION}

Staggered fermion simulations are currently able to reach much lower dynamical quark masses than other fermion discretizations. However, taste-symmetry breaking is numerically significant at current lattice spacings. Thus combined chiral and continuum extrapolations incorporating discretization errors are crucial for correct extrapolation of physical quantities. Moreover, the $\sqrt[4]{\text { Det }}$ trick, which must be in practice be used before taking the continuum limit, 
may or may not change the universality class of the theory. Thus it is unclear whether one is actually studying QCD. We have addressed both of these issues in this paper.

By enumerating all of the NLO operators in the staggered chiral Lagrangian, including source terms, we allow a full NLO calculation, including analytic terms, of PGB properties. Because $S O(4)$-taste symmetry breaking first enters at this order, these operators correctly reflect the true symmetry group of the underlying lattice action. They are therefore necessary, combined with one-loop contributions, for accurate extrapolations and precise determinations of physical quantities.

As we noted in the Introduction, the large number of operators that appear at NLO implies that one must calculate a correspondingly large number of physical quantities in order to obtain predictions. Thus, for most new quantities one calculates (e.g. the decay constants of PGBs of different tastes), the NLO analytic terms simply give to an independent contribution proportional to $a^{2}$. The most important NLO terms are then the one-loop contributions, which lead to non-analytic dependence on the quark mass and lattice spacing.

The exceptions to this observation are quantities which are only non-vanishing because of $S O(4)$ breaking. These only recieve contributions from the NLO operators we have enumerated, and, in fact, from only a handful of these. There are no loop contributions at NLO. This allows us to make a number of testable predictions relating $S O(4)$ breaking splitting in PGB masses, decay constants, and dispersion relations. These predictions can be tested by calculating only two-point correlators of unrenormalized lattice operators. For the hairpin operators we suggest a method similar to that used successfully in quenched simulations. Thus we hope that it will be practical to carry out these tests.

We also find a few predictions for quantities that do not involve $S O(4)$ breaking, but reflect the structure of $\mathrm{S} \chi \mathrm{PT}$. These are NLO analogues of the absence of the taste tensor hairpin at LO. We stress that these predictions, like those above, are not consequences of lattice symmetries, and indeed are violated at NNLO or higher order in the chiral-continuum expansion. In a similar vein, we note that not all operators in the effective chiral theory which are consistent with the lattice symmetries and power counting actually appear at a given order. One must enumerate quark-level operators and then match these onto mesonic operators, and this leads, at NLO, to restrictions on the contraction of indices.

In our view, the most important application of the predictions we have given is to test the applicability of the effective chiral theory when one uses the $\sqrt[4]{\text { Det }}$ trick, and by so doing to test the assumption that this trick does not modify the continuum limit. Most of our predictions are unchanged in form when one implements the $\sqrt[4]{\text { Det }}$ trick by hand in the effective theory. This is both good and bad. It is good because it makes the predictions somewhat more theoretically robust; it is bad because one would like to calculate quantities which directly check the factors of $1 / 4$ put in by hand. In any case, the key point is that if $\mathrm{S} \chi \mathrm{PT}$ fails to describe results from numerical simulations with small quark masses, this would shed serious doubt on the correctness of the $\sqrt[4]{\text { Det }}$ trick. Testing our predictions will thus provide further empirical evidence either for or against the validity of this trick.

\section{Acknowledgments}

We thank Oliver Bär and Claude Bernard for useful discussions. This work was supported in part by the US Department of Energy through grant DE-FG02-96ER40956. R.V. thanks the theory group at Fermilab for hospitality while some of this work was undertaken. 


\section{APPENDIX A: DETERMINATION OF $\mathcal{O}\left(a^{2} p^{2}, a^{2} m, a^{4}\right)$ OPERATORS}

Here we determine all operators in staggered chiral perturbation theory which are NLO in our power counting and arise from discretization errors. We do not discuss the remaining NLO operators, which are of $\mathcal{O}\left(p^{4}, p^{2} m, m^{2}\right)$, since they are unchanged from continuum chiral perturbation theory.

We first discuss the chiral operators of $\mathcal{O}\left(a^{2} p^{2}, a^{2} m\right)$. These arise from a single insertion of the quark-level $\mathcal{O}\left(a^{2}\right)$ operators. Then we turn to the $\mathcal{O}\left(a^{4}\right)$ chiral operators, which require either two insertions of quark-level $\mathcal{O}\left(a^{2}\right)$ operators, or a single insertion of an $\mathcal{O}\left(a^{4}\right)$ operator. We close this subsection with a brief note on operators which do not arise until higher than NLO in our expansion. Finally, we consider the $\mathcal{O}\left(a^{2} p^{2}\right)$ operators involving covariant derivatives acting on taste spurions, which lead to additional contributions to the vector and axial currents.

\section{Single insertion of gluonic operators and fermion bilinears}

Most of the dimension six gluonic operators and fermion bilinears do not break continuum symmetries, and can only generate corrections proportional to terms in the continuum chiral Lagrangian. Thus at NLO they only give rise to the two operators in the LO chiral Lagrangian,

$$
\operatorname{Str}\left(\partial_{\mu} \Sigma \partial_{\mu} \Sigma^{\dagger}\right) \text { and } \operatorname{Str}\left(M^{\dagger} \Sigma+M \Sigma^{\dagger}\right),
$$

multiplied by independent coefficients of size $a^{2}$. In other words, they generate independent $\mathcal{O}\left(a^{2}\right)$ corrections to the parameters $f$ and $\mu$. As we will see, many additional contributions to the two operators in A1 arise from the four-fermion operators discussed in the following sections. However, these, too, can simply be absorbed into the unknown coefficients multiplying each operator.

Here we use mass spurions which transform in the same manner as the $\Sigma$ field under a chiral symmetry transformation:

$$
M \rightarrow L M R^{\dagger}, \quad M^{\dagger} \rightarrow R M^{\dagger} L^{\dagger} .
$$

These spurions also function as sources for the scalar and pseudoscalar densities, with $M=s+i p$, and $s$ and $p$ general Hermitian fields. In the absence of external fields we set $s \rightarrow \mathcal{M}$ and $p \rightarrow 0$.

The remaining two gluonic and bilinear operators violate Euclidean rotation symmetry,

$$
\sum_{\mu} \operatorname{Tr}\left(D_{\mu} F_{\mu \nu} D_{\mu} F_{\mu \nu}\right), \quad \sum_{\mu} \bar{Q}\left(\gamma_{\mu} \otimes 1\right) D_{\mu}^{3} Q
$$

although they are taste symmetric. They can give rise to rotationally non-invariant mesonic operators, but this requires four derivatives [12], e.g.

$$
\sum_{\mu} \operatorname{Str}\left(\partial_{\mu} \Sigma \partial_{\mu} \Sigma^{\dagger} \partial_{\mu} \Sigma \partial_{\mu} \Sigma^{\dagger}\right)
$$

These operators are of $\mathcal{O}\left(a^{2} p^{4}\right)$, and thus NNLO, which is one higher order than we consider here.

\section{Single insertions of operators from $S_{6}^{F F(A)}$}

It is important to remember that the four fermion operators in $S_{6}^{F F(A)}$ are invariant under rotations and $S O(4)$ taste transformations, so they must map onto mesonic operators that also respect these symmetries. For discussion purposes, we divide them according to how they transform under $S U(4 N \mid 4 M)_{L} \times S U(4 N \mid 4 M)_{R}$ chiral rotations.

\section{a. Operators with spin structure $V$ or $A$}

Here we consider mesonic operators arising from single insertions of four-fermion operators of the form $[V \times F]$ and $[A \times F]$, where $F=S, P, T$ indicates the taste of the bilinears. The chiral structure of these operators is

$$
\mathcal{O}_{F}= \pm \sum_{\mu}\left(\overline{Q_{R}}\left(\gamma_{\mu} \otimes F_{R}\right) Q_{R} \pm \overline{Q_{L}}\left(\gamma_{\mu} \otimes F_{L}\right) Q_{L}\right)^{2}
$$




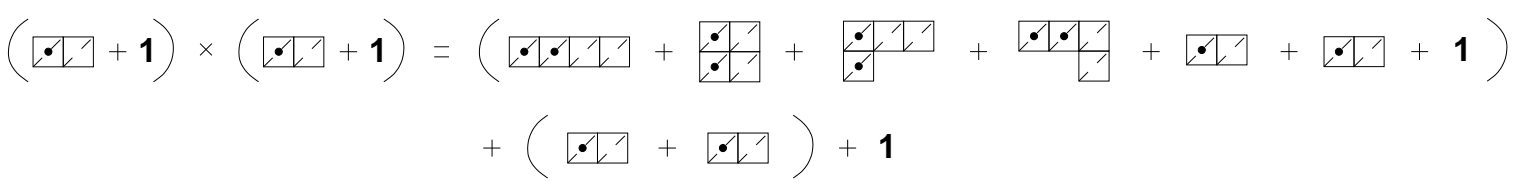

FIG. 3: The product of two bifundamentals in $S U(4 N \mid 4 M)$. The result also applies for $S U(N)$ for $N>3$ (with the dashed lines removed).

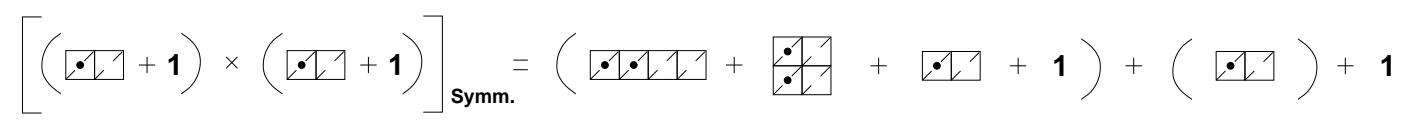

FIG. 4: The symmetric product of two bifundamentals in $S U(4 N \mid 4 M$ ). The result also applies to $S U(N)$ for $N>3$ (with the dashed lines removed).

where and the upper and lower signs correspond to spins $\mathrm{V}$ and $\mathrm{A}$, respectively, and $F_{R, L}$ are Hermitian taste matrices. To determine the corresponding mesonic operators, we promote the taste matrices to spurion fields, with transformations chosen so that $\mathcal{O}_{F}$ is invariant under chiral transformations:

$$
F_{L} \rightarrow L F_{L} L^{\dagger}, \quad F_{R} \rightarrow R F_{R} R^{\dagger}
$$

At the end we will set $F_{L}=F_{R}=F$, where $F$ is the specific taste matrix appearing in the operator.

We first construct the resulting $\mathcal{O}\left(a^{2} p^{2}\right)$ operators. These must be chiral singlets, respect parity $\left(F_{L} \leftrightarrow F_{R}\right.$ and $\Sigma \leftrightarrow \Sigma^{\dagger}$ ), be quadratic in $F$ (odd powers of $F$ are forbidden by the $F \rightarrow-F$ symmetry and quartic terms are higher order in $a$ ), and contain two derivatives. $S_{6}^{F F(A)}$ is rotationally invariant, so the indices of the derivatives must be contracted with each other, and cannot be correlated with any of the indices associated with the taste matrices. It is easiest to construct linearly-independent, chiral singlet operators using elements with the same chiral transformation properties. We use left-handed building blocks, the choices for which are:

$$
\begin{aligned}
F_{L} & \rightarrow L\left(F_{L}\right) L^{\dagger} \\
\Sigma F_{R} \Sigma^{\dagger} & \rightarrow L\left(\Sigma F_{R} \Sigma^{\dagger}\right) L^{\dagger} \\
\Sigma \partial_{\mu} \Sigma^{\dagger} & \rightarrow L\left(\Sigma \partial_{\mu} \Sigma^{\dagger}\right) L^{\dagger}
\end{aligned}
$$

We can now use the graded group theory method of Ref. [36] to determine the number of linearly independent $\mathcal{O}\left(a^{2} p^{2}\right)$ operators in a general $S U(4 N \mid 4 M)$ partially quenched theory. Because all of the operators in (A7) transform as bifundamentals under the left-handed chiral group they all potentially have both adjoint and singlet components. An operator with two taste spurions therefore comes from the product of an adjoint plus singlet times an adjoint plus singlet, as shown in Fig. 3 using the graded Young tableaux notation of Refs. [38, 39]. If the two taste spurions are identical (both $F_{L}$ or $\Sigma F_{R} \Sigma^{\dagger}$ ), it will only come from the symmetric part of this product, shown in Fig. 团 The lefthanded Lie derivative has vanishing supertrace, however, and therefore no singlet component. An operator with two Lie derivatives, which necessarily have their indices contracted and are therefore identical, comes from the symmetric product of two adjoints, shown in Fig. 5 Thus the number of linearly-independent operators is equivalent to the number of singlet representations contained in the product of Fig. [5 which comes from the Lie derivatives, and either Fig. 3 or 4 which comes from the taste spurions.

Consider first operators composed of two Lie Derivatives, one $F_{L}$, and one $\Sigma F_{R} \Sigma^{\dagger}$. This requires counting singlets in the product of the representations in Figs. 3 and 5 . Since all of the representations in Fig. 5 are self-conjugate, each can form a singlet with the identical representation in Fig. 3. This leads to eight linearly-independent operators. We choose the operator basis shown in Table III in which operators have been simplified using the anti-Hermiticity of the Lie derivative and $\Sigma \Sigma^{\dagger}=1$. Each listed operator has the same coefficient with the same sign for both $\mathrm{V}$ and A spins because the overall sign and the cross-term sign in (A5) cancel. Since group theory only considers chiral

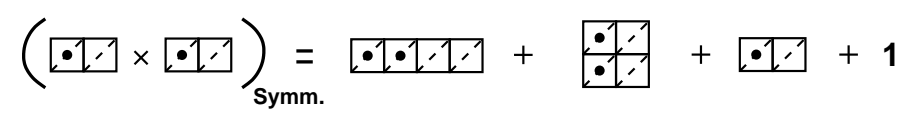

FIG. 5: The symmetric product of two adjoints in $S U(4 N \mid 4 M)$. The undotted boxes are fundamental representations and the dotted boxes are anti-fundamental representations. The result also applies to $S U(N)$ for $N>3$ (with the dashed lines removed). 


\begin{tabular}{lc}
\hline \hline \multicolumn{1}{c}{ Operator } & Keep? \\
\hline 1. $\operatorname{Str}\left(\partial_{\mu} \Sigma^{\dagger} \partial_{\mu} \Sigma \Sigma^{\dagger} F_{L} \Sigma F_{R}\right)$ & Yes - combined with 3 \\
2. $\operatorname{Str}\left(\partial_{\mu} \Sigma^{\dagger} F_{L} \partial_{\mu} \Sigma F_{R}\right)$ & Yes \\
3. $\operatorname{Str}\left(\partial_{\mu} \Sigma \partial_{\mu} \Sigma^{\dagger} \Sigma F_{R} \Sigma^{\dagger} F_{L}\right)$ & Yes - combined with 1 \\
4. $\operatorname{Str}\left(\partial_{\mu} \Sigma \partial_{\mu} \Sigma^{\dagger} F_{L}\right) \operatorname{Str}\left(F_{R}\right)$ & No \\
5. $\operatorname{Str}\left(\partial_{\mu} \Sigma^{\dagger} \partial_{\mu} \Sigma F_{R}\right) \operatorname{Str}\left(F_{L}\right)$ & No \\
6. $\operatorname{Str}\left(\partial_{\mu} \Sigma^{\dagger} \partial_{\mu} \Sigma\right) \operatorname{Str}\left(F_{L} \Sigma F_{R} \Sigma^{\dagger}\right)$ & Yes \\
7. $\operatorname{Str}\left(\Sigma \partial_{\mu} \Sigma^{\dagger} F_{L}\right) \operatorname{Str}\left(\Sigma^{\dagger} \partial_{\mu} \Sigma F_{R}\right)$ & Yes \\
8. $\operatorname{Str}\left(\partial_{\mu} \Sigma^{\dagger} \partial_{\mu} \Sigma\right) \operatorname{Str}\left(F_{L}\right) \operatorname{Str}\left(F_{R}\right)$ & No \\
\hline \hline
\end{tabular}

TABLE III: The eight linearly-independent $\mathcal{O}\left(a^{2} p^{2}\right)$ operators with two derivatives and two different taste spurions. Note that two of them must be combined and some can be neglected because of other considerations, as discussed in the text.

\begin{tabular}{lc}
\hline \hline \multicolumn{1}{c}{ Operator } & Keep? \\
\hline 9. $\pm \operatorname{Str}\left(\partial_{\mu} \Sigma \partial_{\mu} \Sigma^{\dagger} F_{L} F_{L}\right) \pm \operatorname{Str}\left(\partial_{\mu} \Sigma^{\dagger} \partial_{\mu} \Sigma F_{R} F_{R}\right)$ & No \\
10. $\pm \operatorname{Str}\left(\Sigma \partial_{\mu} \Sigma^{\dagger} F_{L} \Sigma \partial_{\mu} \Sigma^{\dagger} F_{L}\right) \pm \operatorname{Str}\left(\Sigma^{\dagger} \partial_{\mu} \Sigma F_{R} \Sigma^{\dagger} \partial_{\mu} \Sigma F_{R}\right)$ & Yes \\
11. $\pm \operatorname{Str}\left(\partial_{\mu} \Sigma^{\dagger} \partial_{\mu} \Sigma\right) \operatorname{Str}\left(F_{L} F_{L}\right) \pm \operatorname{Str}\left(\partial_{\mu} \Sigma^{\dagger} \partial_{\mu} \Sigma\right) \operatorname{Str}\left(F_{R} F_{R}\right)$ & No \\
12. $\pm \operatorname{Str}\left(\partial_{\mu} \Sigma \partial_{\mu} \Sigma^{\dagger} F_{L}\right) \operatorname{Str}\left(F_{L}\right) \pm \operatorname{Str}\left(\partial_{\mu} \Sigma^{\dagger} \partial_{\mu} \Sigma F_{R}\right) \operatorname{Str}\left(F_{R}\right)$ & No \\
13. $\pm \operatorname{Str}\left(\Sigma \partial_{\mu} \Sigma^{\dagger} F_{L}\right) \operatorname{Str}\left(\Sigma \partial_{\mu} \Sigma^{\dagger} F_{L}\right) \pm \operatorname{Str}\left(\Sigma^{\dagger} \partial_{\mu} \Sigma F_{R}\right) \operatorname{Str}\left(\Sigma^{\dagger} \partial_{\mu} \Sigma F_{R}\right)$ & Yes \\
14. $\pm \operatorname{Str}\left(\partial_{\mu} \Sigma^{\dagger} \partial_{\mu} \Sigma\right) \operatorname{Str}\left(F_{L}\right) \operatorname{Str}\left(F_{L}\right) \pm \operatorname{Str}\left(\partial_{\mu} \Sigma^{\dagger} \partial_{\mu} \Sigma\right) \operatorname{Str}\left(F_{R}\right) \operatorname{Str}\left(F_{R}\right)$ & No \\
\hline \hline
\end{tabular}

TABLE IV: The six linearly-independent $\mathcal{O}\left(a^{2} p^{2}\right)$ operators with two derivatives and two identical taste spurions. The upper signs correspond to spin $V$, while the lower signs correspond to spin $A$. Some operators can be neglected because of other considerations, as discussed in the text.

transformation properties, we must impose other symmetries by hand. The operators $1 \& 3$ and $4 \& 5$ transform into each other under parity, so we include their sums, each with a single undetermined coefficient, in the staggered chiral Lagrangian. Operators 4,5 , and 8 are all proportional to $\operatorname{Str}(F)$, so they vanish unless $F=\xi_{I}$. When this is the case, however, they are proportional to the LO kinetic term, which we have already included in the previous sub-section.

Similarly, six linearly-independent operators can be made out of two Lie Derivatives and two $F_{L}$ 's or $F_{R}$ 's. These are shown in Table IV Here the minus signs in A5 do not cancel, and the coefficients of operators with $V$ and $A$ spins have the same magnitude, but opposite sign. Operator 12 vanishes unless $F=\xi_{I}$, and then is proportional to the LO kinetic term, as are operators 9, 11, and 14 for all tastes, so we can neglect them. After combining both lists, we are left with six new linearly independent $\mathcal{O}\left(a^{2} p^{2}\right)$ operators.

We note in passing that the fourteen operators in Tables III and IV are those obtained simply by building operators out of the appropriate elements and using the cyclicity of the supertrace. There are no relationships between these operators in the PQ theory, unlike for chiral $S U(2)$ and $S U(3)$ theories. Typically, such relationships are found using Cayley-Hamilton relations, as in Refs. [40] and [41]. There is, however, no graded analog of Cayley-Hamilton relations, since the superdeterminant is not a finite polynomial [39].

To obtain the actual operators in the chiral Lagrangian we set $F_{L}=F_{R}=F$, a specific taste matrix, here $S, P$ or $T$. Since rotational and $S O(4)$ taste symmetry are unbroken, the indices on the derivatives must be contracted together, as must those on the taste matrices. With these rules, it is easy to see that the specific $\mathcal{O}\left(a^{2} p^{2}\right)$ operators in the $\mathrm{S} \chi \mathcal{L}$ generated by operators in $S^{F F(A)}$ with spins $V$ and $A$ are those shown in Table XVII We have dropped the \pm signs at this stage because we do not know the relative size of the coefficients of the underlying four-fermion operators with spins $\mathrm{V}$ and $\mathrm{A}$, and therefore cannot make use of the fact that their mappings into the chiral Lagrangian are related. We have also dropped the operators resulting from taste $S$, since they are either of the same form as the LO chiral Lagrangian, or vanish because $\operatorname{Str}\left(\Sigma \partial_{\mu} \Sigma^{\dagger}\right)=0$.

We determine the $\mathcal{O}\left(a^{2} m\right)$ operators using the same methodology. We use the mass spurions introduced above, which lead to two new left-handed objects:

$$
M \Sigma^{\dagger} \rightarrow L M \Sigma^{\dagger} L^{\dagger}, \quad \Sigma M^{\dagger} \rightarrow L \Sigma M^{\dagger} L^{\dagger} .
$$

Like the taste spurions, these objects are also bifundamentals under the left-handed chiral group. Operators of order 


\begin{tabular}{lc}
\hline \hline \multicolumn{1}{c}{ Operator } & Keep? \\
\hline 15. $\operatorname{Str}\left(F_{L} \Sigma F_{R} M^{\dagger}\right)$ & Yes - combined with 22 \\
16. $\operatorname{Str}\left(F_{L} \Sigma M^{\dagger} \Sigma F_{R} \Sigma^{\dagger}\right)$ & Yes - combined with 21 \\
17. $\operatorname{Str}\left(F_{L} \Sigma F_{R} \Sigma^{\dagger}\right) \operatorname{Str}\left(\Sigma M^{\dagger}\right)$ & Yes - combined with 23 \\
18. $\operatorname{Str}\left(F_{L} \Sigma M^{\dagger}\right) \operatorname{Str}\left(F_{R}\right)$ & No \\
19. $\operatorname{Str}\left(F_{L}\right) \operatorname{Str}\left(F_{R} M^{\dagger} \Sigma\right)$ & No \\
20. $\operatorname{Str}\left(F_{L}\right) \operatorname{Str}\left(F_{R}\right) \operatorname{Str}\left(\Sigma M^{\dagger}\right)$ & No \\
21. $\operatorname{Str}\left(F_{R} \Sigma^{\dagger} M \Sigma^{\dagger} F_{L} \Sigma\right)$ & Yes - combined with 16 \\
22. $\operatorname{Str}\left(F_{R} \Sigma^{\dagger} F_{L} M\right)$ & Yes - combined with 15 \\
23. $\operatorname{Str}\left(F_{L} \Sigma F_{R} \Sigma^{\dagger}\right) \operatorname{Str}\left(M \Sigma^{\dagger}\right)$ & Yes - combined with 17 \\
24. $\operatorname{Str}\left(F_{L} M \Sigma^{\dagger}\right) \operatorname{Str}\left(F_{R}\right)$ & No \\
25. $\operatorname{Str}\left(F_{L}\right) \operatorname{Str}\left(F_{R} \Sigma^{\dagger} M\right)$ & No \\
26. $\operatorname{Str}\left(F_{L}\right) \operatorname{Str}\left(F_{R}\right) \operatorname{Str}\left(M \Sigma^{\dagger}\right)$ & No \\
\hline \hline
\end{tabular}

TABLE V: The twelve linearly-independent $\mathcal{O}\left(a^{2} m\right)$ operators one mass spurion and two different taste spurions. Many can be neglected, and the rest must be combined because of symmetry considerations.

\begin{tabular}{lc}
\hline \hline \multicolumn{1}{c}{ Operator } & Keep? \\
\hline 27. $\pm \operatorname{Str}\left(F_{L} F_{L} \Sigma M^{\dagger}\right) \pm \operatorname{Str}\left(F_{R} F_{R} \Sigma^{\dagger} M\right)$ & No \\
28. $\pm \operatorname{Str}\left(F_{L} F_{L}\right) \operatorname{Str}\left(\Sigma M^{\dagger}\right) \pm \operatorname{Str}\left(F_{R} F_{R}\right) \operatorname{Str}\left(\Sigma^{\dagger} M\right)$ & No \\
29. $\pm \operatorname{Str}\left(F_{L}\right) \operatorname{Str}\left(F_{L} \Sigma M^{\dagger}\right) \pm \operatorname{Str}\left(F_{R}\right) \operatorname{Str}\left(F_{R} \Sigma^{\dagger} M\right)$ & No \\
30. $\pm \operatorname{Str}\left(F_{L}\right) \operatorname{Str}\left(F_{L}\right) \operatorname{Str}\left(\Sigma M^{\dagger}\right) \pm \operatorname{Str}\left(F_{R}\right) \operatorname{Str}\left(F_{R}\right) \operatorname{Str}\left(\Sigma^{\dagger} M\right)$ & No \\
31. $\pm \operatorname{Str}\left(F_{L} F_{L} M \Sigma^{\dagger}\right) \pm \operatorname{Str}\left(F_{R} F_{R} M^{\dagger} \Sigma\right)$ & No \\
32. $\pm \operatorname{Str}\left(F_{L} F_{L}\right) \operatorname{Str}\left(M \Sigma^{\dagger}\right) \pm \operatorname{Str}\left(F_{R} F_{R}\right) \operatorname{Str}\left(M^{\dagger} \Sigma\right)$ & No \\
33. $\pm \operatorname{Str}\left(F_{L}\right) \operatorname{Str}\left(F_{L} M \Sigma^{\dagger}\right) \pm \operatorname{Str}\left(F_{R}\right) \operatorname{Str}\left(F_{R} M^{\dagger} \Sigma\right)$ & No \\
34. $\pm \operatorname{Str}\left(F_{L}\right) \operatorname{Str}\left(F_{L}\right) \operatorname{Str}\left(M \Sigma^{\dagger}\right) \pm \operatorname{Str}\left(F_{R}\right) \operatorname{Str}\left(F_{R}\right) \operatorname{Str}\left(M^{\dagger} \Sigma\right)$ & No \\
\hline \hline
\end{tabular}

TABLE VI: The eight linearly-independent $\mathcal{O}\left(a^{2} m\right)$ operators with one mass spurion and two identical taste spurions. The upper signs correspond to spin $V$, while the lower signs correspond to spin $A$. All of these operators can be neglected because of additional considerations.

$\mathcal{O}\left(a^{2} m\right)$ will contain two taste spurions and one mass spurion, and be chiral singlets that respect parity. They will therefore come from the singlet representations contained in the product of three bifundamentals. Such operators may contain either two identical or two different taste spurions.

If the taste spurions are different, the operators simply come from the product of two bifundamentals, Figure 3 with a third, which clearly contains six singlets. However, because there are two types of mass spurions, there are twelve corresponding operators in Table $\mathbb{\nabla}$ Each of these operators must be combined with its parity conjugate to form a parity-invariant operator, so there are in fact only six operators in the chiral Lagrangian: $(15+22),(16+$ $21),(17+23),(18+25),(19+24)$, and $(20+26)$. Three of these operators are proportional to $\operatorname{Str}(F)$, and so vanish except for taste $S$, but then reduce to the LO operator $\operatorname{Str}\left(\Sigma M^{\dagger}+M \Sigma^{\dagger}\right)$. Thus we are left with only three new $\mathcal{O}\left(a^{2} m\right)$ operators.

In fact, only two of these three are new if we consider S-matrix elements. Operator $(15+22)$ reduces to the LO operator if $M$ is set to the mass matrix, since $\mathcal{M}$ then commutes with $F_{L}$ and $F_{R}$. The operator does, however, contribute $S U(4)$ taste breaking contributions to pseudoscalar matrix elements, for then $M$ is a source and need not be proportional to the identity in taste space.

If the operators contain identical taste spurions, two of the three bifundamentals must be symmetrized, as shown in Figure [5] The overall product contains four singlets, and therefore generates the eight corresponding operators in Table VI However, using the fact that $F^{2}=1$ for all tastes as well as $\operatorname{Str}(F)=0$ except for $\xi_{I}$, it is easy to see that all of these operators reduce to $\operatorname{Str}\left(\Sigma M^{\dagger}+M \Sigma^{\dagger}\right)$ and can be neglected.

Turning these generic structures into operators in the staggered chiral Lagrangian is now straightforward: simply let $F_{L}=F_{R}=F$. We do not set $M=M^{\dagger}=\mathcal{M}$, however, so as to allow the derivation of (pseudo)scalar matrix 
elements. The linearly-independent $\mathcal{O}\left(a^{2} m\right)$ operators corresponding to $S_{6}^{F F(A)}$ operators with spins $V$ and $A$ are shown in Table XVII

Finally, we briefly recall the construction of the LO operators of $\mathcal{O}\left(a^{2}\right)$, so as to make contact with the discussion in the main text. These operators contain two taste spurions, and the only non-trivial $S U(N \mid M)$ singlet is

$$
\operatorname{Str}\left(F_{L} \Sigma F_{R} \Sigma^{\dagger}\right)
$$

This leads to the operators $\mathcal{O}_{1}$ and $\mathcal{O}_{6}$ in the potential $\mathcal{U}$ in (18).

\section{b. Operators with spin structure $S$ or $P$}

Here we consider single insertions of operators $[S \times F]$ and $[P \times F]$, where the taste can be $F=V$ or $A$. The chiral structure of these operators is

$$
\mathcal{O}_{F}^{\prime}=\left(\overline{Q_{L}}\left(1 \otimes \widetilde{F}_{L}\right) Q_{R} \pm \overline{Q_{R}}\left(1 \otimes \widetilde{F}_{R}\right) Q_{L}\right)^{2}
$$

where and the upper and lower signs correspond to spin $\mathrm{S}$ and $\mathrm{P}$, respectively, and $\widetilde{F}_{L, R}$ are taste matrices. To determine the corresponding mesonic operators in the $\mathrm{S} \chi \mathcal{L}$, we promote the taste matrices to taste spurion fields transforming as

$$
\widetilde{F}_{L} \rightarrow L \widetilde{F}_{L} R^{\dagger}, \quad \widetilde{F}_{R} \rightarrow R \widetilde{F}_{R} L^{\dagger}
$$

Note that these transformations do not maintain the Hermiticity of the original taste matrices. We can, however, consistently impose the relation $F_{L}^{\dagger}=F_{R}$, and so do not need to consider $F_{L, R}^{\dagger}$ as additional variables. The left-handed basis for constructing the mesonic operators becomes

$$
\widetilde{F}_{L} \Sigma^{\dagger}, \quad \Sigma \widetilde{F}_{R}, \quad \Sigma \partial_{\mu} \Sigma^{\dagger}, \quad M \Sigma^{\dagger}, \quad \Sigma M^{\dagger}
$$

The counting and construction of operators with the correct taste structure is identical to the $[V, A \times F]$ case, although the taste spurions are different. The primary technical difference arises when we set $\widetilde{F}_{L}=\widetilde{F}_{R}=\widetilde{F}$, because the relative minus signs within compound operators are different.

As before, there are fourteen $\mathcal{O}\left(a^{2} p^{2}\right)$ operators which can be constructed from two taste spurions and two Lie derivatives. These are shown in Table VII Notice that the \pm factors differ from those for $[V, A \times F]$ because (A10) only has an internal minus sign, not an overall one. Two pairs of operators, $35 \& 37$ and 38 \& 39 , transform into each other under parity, so we include their sums, each with a single coefficient, in the chiral Lagrangian. Operators $(35+37)$ and 40 both reduce to the LO kinetic operator after setting $\widetilde{F}_{L}=\widetilde{F}_{R}=\widetilde{F}$. This leaves ten new linearly independent $\mathcal{O}\left(a^{2} p^{2}\right)$ operators.

In addition, there are twenty $\mathcal{O}\left(a^{2} m\right)$ operators that contain one mass and two taste spurions. These are listed in Table VIII Six pairs of operators transform into each other under parity, and must therefore be included in the Lagrangian with a single coefficient: $49 \& 56,50 \& 55,51 \& 57,52 \& 59,53 \& 58$, and 54 \& 60 . However, operators $(49+56),(50+55)$, and $(51+57)$ reduce to the LO mass term after setting $\widetilde{F}_{L}=\widetilde{F}_{R}=\widetilde{F}$. Thus there are eleven new linearly-independent, parity-invariant $\mathcal{O}\left(a^{2} m\right)$ operators. Three of these, $(52+59)$, 61, and 63 , are pure source terms, however, since they reduce to LO operators when $M=M^{\dagger}=\mathcal{M}$.

To turn these generic taste structures into actual operators in the $\mathrm{S} \chi \mathcal{L}$, we set $\widetilde{F}_{L}=\widetilde{F}_{R}=\widetilde{F}$, with $\widetilde{F}$ being either $V$ or $A$. However, we choose to leave $M$ and $M^{\dagger}$ as generic sources which can be set to the quark mass matrix when necessary. As before must contract indices on derivatives and taste matrices separately, so as not to break rotational or $S O(4)$ taste symmetry. The resulting operators are listed in Table XVIII]

Finally, we recall the construction of the LO operators of $\mathcal{O}\left(a^{2}\right)$. In this case there are three non-trivial $S U(N \mid M)$ singlets:

$$
\begin{aligned}
& \operatorname{Str}\left(\widetilde{F}_{L} \Sigma^{\dagger} \widetilde{F}_{L} \Sigma^{\dagger}\right)+\text { p.c. } \\
& \operatorname{Str}\left(\widetilde{F}_{L} \Sigma^{\dagger}\right) \operatorname{Str}\left(\widetilde{F}_{L} \Sigma^{\dagger}\right)+\text { p.c. } \\
& \operatorname{Str}\left(\widetilde{F}_{L} \Sigma^{\dagger}\right) \operatorname{Str}\left(\Sigma \widetilde{F}_{R}\right) .
\end{aligned}
$$

The first of these leads to $\mathcal{O}_{3}$ and $\mathcal{O}_{4}$ in $\mathcal{U}$, (18), the second to $\mathcal{O}_{2 V}$ and $\mathcal{O}_{2 A}$ in $\mathcal{U}^{\prime}$, (19), and the third to $\mathcal{O}_{5 A}$ and $\mathcal{O}_{5 B}$ in $\mathcal{U}^{\prime}$. 


\begin{tabular}{lc}
\hline \hline \multicolumn{1}{c}{ Operator } & Keep? \\
\hline 35. $\pm \operatorname{Str}\left(\partial_{\mu} \Sigma \partial_{\mu} \Sigma^{\dagger} \widetilde{F}_{L} \widetilde{F}_{R}\right)$ & No \\
36. $\pm \operatorname{Str}\left(\Sigma \partial_{\mu} \Sigma^{\dagger} \widetilde{F}_{L} \Sigma^{\dagger} \partial_{\mu} \Sigma \widetilde{F}_{R}\right)$ & Yes \\
37. $\pm \operatorname{Str}\left(\partial_{\mu} \Sigma^{\dagger} \partial_{\mu} \Sigma \widetilde{F}_{R} \widetilde{F}_{L}\right)$ & No \\
38. $\pm \operatorname{Str}\left(\partial_{\mu} \Sigma^{\dagger} \partial_{\mu} \Sigma^{\dagger} \widetilde{F}_{L}\right) \operatorname{Str}\left(\Sigma \widetilde{F}_{R}\right)$ & Yes - combined with 39 \\
39. $\pm \operatorname{Str}\left(\partial_{\mu} \Sigma \partial_{\mu} \Sigma^{\dagger} \Sigma \widetilde{F}_{R}\right) \operatorname{Str}\left(\widetilde{F}_{L} \Sigma^{\dagger}\right)$ & Yes - combined with 38 \\
40. $\pm \operatorname{Str}\left(\partial_{\mu} \Sigma^{\dagger} \partial_{\mu} \Sigma\right) \operatorname{Str}\left(\widetilde{F}_{L} \widetilde{F}_{R}\right)$ & No \\
41. $\pm \operatorname{Str}\left(\partial_{\mu} \Sigma^{\dagger} \widetilde{F}_{L}\right) \operatorname{Str}\left(\partial_{\mu} \Sigma \widetilde{F}_{R}\right)$ & Yes \\
42. $\pm \operatorname{Str}\left(\partial_{\mu} \Sigma^{\dagger} \partial_{\mu} \Sigma\right) \operatorname{Str}\left(\widetilde{F}_{L} \Sigma^{\dagger}\right) \operatorname{Str}\left(\Sigma \widetilde{F}_{R}\right)$ & Yes \\
43. $\operatorname{Str}\left(\partial_{\mu} \Sigma \partial_{\mu} \Sigma^{\dagger} \widetilde{F}_{L} \Sigma^{\dagger} \widetilde{F}_{L} \Sigma^{\dagger}\right)+\operatorname{Str}\left(\partial_{\mu} \Sigma^{\dagger} \partial_{\mu} \Sigma \widetilde{F}_{R} \Sigma \widetilde{F}_{R} \Sigma\right)$ & Yes \\
44. $\operatorname{Str}\left(\partial_{\mu} \Sigma^{\dagger} \widetilde{F}_{L} \partial_{\mu} \Sigma^{\dagger} \widetilde{F}_{L}\right)+\operatorname{Str}\left(\partial_{\mu} \Sigma \widetilde{F}_{R} \partial_{\mu} \Sigma \widetilde{F}_{R}\right)$ & Yes \\
45. $\operatorname{Str}\left(\partial_{\mu} \Sigma^{\dagger} \partial_{\mu} \Sigma\right) \operatorname{Str}\left(\widetilde{F}_{L} \Sigma^{\dagger} \widetilde{F}_{L} \Sigma^{\dagger}\right)+\operatorname{Str}\left(\partial_{\mu} \Sigma^{\dagger} \partial_{\mu} \Sigma\right) \operatorname{Str}\left(\Sigma \widetilde{F}_{R} \Sigma \widetilde{F}_{R}\right)$ & Yes \\
46. $\operatorname{Str}\left(\partial_{\mu} \Sigma \partial_{\mu} \Sigma^{\dagger} \widetilde{F}_{L} \Sigma^{\dagger}\right) \operatorname{Str}\left(\widetilde{F}_{L} \Sigma^{\dagger}\right)+\operatorname{Str}\left(\partial_{\mu} \Sigma^{\dagger} \partial_{\mu} \Sigma \widetilde{F}_{R} \Sigma\right) \operatorname{Str}\left(\Sigma \widetilde{F}_{R}\right)$ & Yes \\
47. $\operatorname{Str}\left(\partial_{\mu} \Sigma^{\dagger} \widetilde{F}_{L}\right) \operatorname{Str}\left(\partial_{\mu} \Sigma^{\dagger} \widetilde{F}_{L}\right)+\operatorname{Str}\left(\partial_{\mu} \Sigma \widetilde{F}_{R}\right) \operatorname{Str}\left(\partial_{\mu} \Sigma \widetilde{F}_{R}\right)$ & Yes \\
48. $\operatorname{Str}\left(\partial_{\mu} \Sigma^{\dagger} \partial_{\mu} \Sigma\right) \operatorname{Str}\left(\widetilde{F}_{L} \Sigma^{\dagger}\right) \operatorname{Str}\left(\widetilde{F}_{L} \Sigma^{\dagger}\right)+\operatorname{Str}\left(\partial_{\mu} \Sigma^{\dagger} \partial_{\mu} \Sigma\right) \operatorname{Str}\left(\Sigma \widetilde{F}_{R}\right) \operatorname{Str}\left(\Sigma \widetilde{F}_{R}\right)$ & Yes \\
\hline \hline
\end{tabular}

TABLE VII: The fourteen linearly-independent $\mathcal{O}\left(a^{2} p^{2}\right)$ operators with two derivatives and two taste spurions. When present, the upper and lower signs correspond to spins $S$ and $P$, respectively.

\section{c. Operators with spin structure $T$}

The spin $T$ operators, $[T \times V]$ and $[T \times A]$, have almost the same chiral structure as those of spins $S$ and $P$ :

$$
\mathcal{O}_{F}^{\prime \prime}=\sum_{\mu<\nu}\left(\overline{Q_{L}}\left(\gamma_{\mu \nu} \otimes \widetilde{F}_{L}\right) Q_{R}\right)^{2}+\left(\overline{Q_{R}}\left(\gamma_{\nu \mu} \otimes \widetilde{F}_{R}\right) Q_{L}\right)^{2}
$$

The only difference is that there are no cross terms between $\widetilde{F}_{L}$ and $\widetilde{F}_{R}$. This means that the corresponding chiral operators are identical to those generated by $[S, P \times V, A]$, except that those coming from $\widetilde{F}_{L} \widetilde{F}_{R}$ cross-terms are absent. Thus the only effect of single insertions of four-fermion operators with spin structure $T$ is to change the (unknown) coefficients of some of the mesonic operators already listed.

\section{Single insertions of operators from $S_{6}^{F F(B)}$}

The four-fermion operators in $S_{6}^{F F(B)}$ break both rotational symmetry and the remaining $S O(4)$ taste symmetry repected by operators in $S_{6}^{F F(A)}$. Thus they can map onto mesonic operators that also break these symmetries. Indeed they only map onto such operators, because they are constructed to have no taste singlet component. In this subsection we construct all such operators resulting from a single insertion of $S_{6}^{F F(B)}$.

We begin with a general comment. To break the rotational and/or $S O(4)$ taste symmetries requires that the mesonic operator have more than two repeated indices. This can be accomplished at NLO either by having two derivatives and two taste spurions, or by having four taste spurions. The former choice leads to the $\mathcal{O}\left(a^{2} p^{2}\right)$ operators exemplified by

$$
\sum_{\mu} \operatorname{Str}\left(\Sigma \partial_{\mu} \Sigma^{\dagger} \xi_{\mu} \Sigma^{\dagger} \partial_{\mu} \Sigma \xi_{\mu}\right)
$$

and discussed in this subsection. The latter choice gives $\mathcal{O}\left(a^{4}\right)$ operators such as

$$
\sum_{\mu} \sum_{\nu \neq \mu} \operatorname{Str}\left(\xi_{\mu \nu} \Sigma \xi_{\mu} \Sigma \xi_{\nu \mu} \Sigma^{\dagger} \xi_{\mu} \Sigma^{\dagger}\right)
$$

which will be discussed separately below. It is not possible, however, to break the symmetries with two taste spurions and a mass spurion. Thus there are no new $\mathcal{O}\left(a^{2} m\right)$ operators produced by $S_{6}^{F F(B)}$. 


\begin{tabular}{lc}
\hline \hline & \multicolumn{1}{c}{ Operator } \\
\hline 49. $\pm \operatorname{Str}\left(\widetilde{F}_{L} \widetilde{F}_{R} \Sigma M^{\dagger}\right)$ & Keep? \\
50. $\pm \operatorname{Str}\left(\widetilde{F}_{L} M^{\dagger} \Sigma \widetilde{F}_{R}\right)$ & No \\
51. $\pm \operatorname{Str}\left(\widetilde{F}_{L} \widetilde{F}_{R}\right) \operatorname{Str}\left(\Sigma M^{\dagger}\right)$ & No \\
52. $\pm \operatorname{Str}\left(\widetilde{F}_{L} M^{\dagger}\right) \operatorname{Str}\left(\Sigma \widetilde{F}_{R}\right)$ & Yes - combined with 59 \\
53. $\pm \operatorname{Str}\left(\widetilde{F}_{L} \Sigma^{\dagger}\right) \operatorname{Str}\left(\Sigma \widetilde{F}_{R} \Sigma M^{\dagger}\right)$ & Yes - combined with 58 \\
54. $\pm \operatorname{Str}\left(\widetilde{F}_{L} \Sigma^{\dagger}\right) \operatorname{Str}\left(\Sigma \widetilde{F}_{R}\right) \operatorname{Str}\left(\Sigma M^{\dagger}\right)$ & Yes - combined with 60 \\
55. $\pm \operatorname{Str}\left(\widetilde{F}_{L} \widetilde{F}_{R} M \Sigma^{\dagger}\right)$ & No \\
56. $\pm \operatorname{Str}\left(\widetilde{F}_{L} \Sigma^{\dagger} M \widetilde{F}_{R}\right)$ & No \\
57. $\pm \operatorname{Str}\left(\widetilde{F}_{L} \widetilde{F}_{R}\right) \operatorname{Str}\left(M \Sigma^{\dagger}\right)$ & No \\
58. $\pm \operatorname{Str}\left(\widetilde{F}_{L} \Sigma^{\dagger} M \Sigma^{\dagger}\right) \operatorname{Str}\left(\Sigma \widetilde{F}_{R}\right)$ & Yes - combined with 53 \\
59. $\pm \operatorname{Str}\left(\widetilde{F}_{L} \Sigma^{\dagger}\right) \operatorname{Str}\left(\widetilde{F}_{R} M\right)$ & Yes - combined with 53 \\
60. $\pm \operatorname{Str}\left(\widetilde{F}_{L} \Sigma^{\dagger}\right) \operatorname{Str}\left(\Sigma \widetilde{F}_{R}\right) \operatorname{Str}\left(M \Sigma^{\dagger}\right)$ & Yes - combined with 54 \\
61. $\operatorname{Str}\left(\widetilde{F}_{L} \Sigma^{\dagger} \widetilde{F}_{L} M^{\dagger}\right)+\operatorname{Str}\left(\widetilde{F}_{R} \Sigma \widetilde{F}_{R} M\right)$ & Yes \\
62. $\operatorname{Str}\left(\widetilde{F}_{L} \Sigma^{\dagger} \widetilde{F}_{L} \Sigma^{\dagger}\right) \operatorname{Str}\left(\Sigma M^{\dagger}\right)+\operatorname{Str}\left(\widetilde{F}_{R} \Sigma \widetilde{F}_{R} \Sigma\right) \operatorname{Str}\left(\Sigma^{\dagger} M\right)$ & Yes \\
63. $\operatorname{Str}\left(\widetilde{F}_{L} \Sigma^{\dagger}\right) \operatorname{Str}\left(\widetilde{F}_{L} M^{\dagger}\right)+\operatorname{Str}\left(\widetilde{F}_{R} \Sigma\right) \operatorname{Str}\left(\widetilde{F}_{R} M\right)$ & Yes \\
64. $\operatorname{Str}\left(\widetilde{F}_{L} \Sigma^{\dagger}\right) \operatorname{Str}\left(\widetilde{F}_{L} \Sigma^{\dagger}\right) \operatorname{Str}\left(\Sigma M{ }^{\dagger}\right)+\operatorname{Str}\left(\widetilde{F}_{R} \Sigma\right) \operatorname{Str}\left(\widetilde{F}_{R} \Sigma\right) \operatorname{Str}\left(\Sigma^{\dagger} M\right)$ & Yes \\
65. $\operatorname{Str}\left(\widetilde{F}_{L} \Sigma^{\dagger} \widetilde{F}_{L} \Sigma^{\dagger} M \Sigma^{\dagger}\right)+\operatorname{Str}\left(\widetilde{F}_{R} \Sigma \widetilde{F}_{R} \Sigma M^{\dagger} \Sigma\right)$ & Yes \\
66. $\operatorname{Str}\left(\widetilde{F}_{L} \Sigma^{\dagger} \widetilde{F}_{L} \Sigma^{\dagger}\right) \operatorname{Str}\left(M \Sigma^{\dagger}\right)+\operatorname{Str}\left(\widetilde{F}_{R} \Sigma \widetilde{F}_{R} \Sigma\right) \operatorname{Str}\left(M^{\dagger} \Sigma\right)$ & Yes \\
67. $\operatorname{Str}\left(\widetilde{F}_{L} \Sigma^{\dagger}\right) \operatorname{Str}\left(\widetilde{F}_{L} \Sigma^{\dagger} M \Sigma^{\dagger}\right)+\operatorname{Str}\left(\widetilde{F}_{R} \Sigma\right) \operatorname{Str}\left(\widetilde{F}_{R} \Sigma M^{\dagger} \Sigma\right)$ & Yes \\
68. $\operatorname{Str}\left(\widetilde{F}_{L} \Sigma^{\dagger}\right) \operatorname{Str}\left(\widetilde{F}_{L} \Sigma^{\dagger}\right) \operatorname{Str}\left(M \Sigma^{\dagger}\right)+\operatorname{Str}\left(\widetilde{F}_{R} \Sigma\right) \operatorname{Str}\left(\widetilde{F}_{R} \Sigma\right) \operatorname{Str}\left(M^{\dagger} \Sigma\right)$ & Yes \\
\hline \hline & \\
\hline
\end{tabular}

TABLE VIII: The twenty linearly-independent $\mathcal{O}\left(a^{2} m\right)$ operators with one mass spurion and two taste spurions. When present, the upper signs correspond to spin $S$, while the lower signs correspond to spin $P$.

Although the operators in $S_{6}^{F F(B)}$ appear more complicated than those in $S_{6}^{F F(A)}$, because of the coupled spin and taste matrices, we can actually reuse the bulk of our work from the previous subsection. This is because we can still use spurion analysis and group theory to determine the chiral structure of the mesonic operators. We just need to be more careful about the index structure and the specific taste matrices. We illustrate the procedure by working through the mapping of $\left[V_{\mu} \times T_{\mu}\right]$ in detail; the method then can be generalized straighforwardly to the remaining operators in $S_{6}^{F F(B)}$.

We begin by recalling the definition of this operator:

$$
\left[V_{\mu} \times T_{\mu}\right] \equiv \sum_{\mu} \sum_{\nu \neq \mu}\left\{\bar{Q}\left(\gamma_{\mu} \otimes \xi_{\mu \nu}\right) Q \bar{Q}\left(\gamma_{\mu} \otimes \xi_{\nu \mu}\right) Q-\bar{Q}\left(\gamma_{\mu} \otimes \xi_{\mu \nu 5}\right) Q \bar{Q}\left(\gamma_{\mu} \otimes \xi_{5 \nu \mu}\right) Q\right\} .
$$

The second term in this expression removes the taste singlet component. It is, however, cumbersome and unnecessary to keep both terms, so we first simplify using

$$
\frac{1}{2}\left(\left[V_{\mu} \times T_{\mu}\right]+[V \times T]\right) \equiv \sum_{\mu} \sum_{\nu \neq \mu} \bar{Q}\left(\gamma_{\mu} \otimes \xi_{\mu \nu}\right) Q \bar{Q}\left(\gamma_{\mu} \otimes \xi_{\nu \mu}\right) Q
$$

Since $[V \times T]$ is from $S_{6}^{F F(A)}$ this addition has no impact on matching to operators which break rotation and/or taste $S O(4)$ symmetries. The chiral structure of the operator in (A20) is given by

$$
\mathcal{O}(\mu)_{F}= \pm\left[\overline{Q_{R}}\left(\gamma_{\mu} \otimes F(\mu)_{R}\right) Q_{R} \pm \overline{Q_{L}}\left(\gamma_{\mu} \otimes F(\mu)_{L}\right) Q_{L}\right]^{2}
$$

which has the same form as for $[V \times T]$, A5 , except that the taste spurions depend on $\mu$, and the sum over $\mu$ has been removed for now. The mapping then proceeds as follows: first, determine the mapping of $\mathcal{O}(\mu)_{F}$ onto mesonic operators, for fixed $\mu$; next, set the taste spurions to their appropriate values; and, finally, sum over $\mu$ and any other remaining indices.

The group theory required to determine the independent mappings of $\mathcal{O}(\mu)_{F}$ onto mesonic operators is identical to that for $[V \times T]$, since the spurions $F(\mu)_{R, L}$ must transform like $F_{R, L}$. Thus one obtains the types enumerated 
in Tables $\llbracket$ and IV with the provisos that $F_{R, L} \longrightarrow F(\mu)_{R, L}$ and that we must be careful with Lorentz (or, more precisely, hypercubic group) indices. In particular, we do not yet know how the indices $\mu$ in the operators in the tables are connected with the index $\mu$ in $\mathcal{O}(\mu)_{F}$. To work this out we need to determine how $\mathcal{O}(\mu)_{F}$ transforms under rotations while keeping the spurions fixed. This may seem confusing because of the index $\mu$ in $F(\mu)_{R, L}$, but once, for a given $\mu$, we convert the taste matrices into spurion fields, they are to be treated as rotationally-invariant (pseudo)scalar fields. Then, since $\mathcal{O}(\mu)_{F}$ has two vector indices, it has a component which is a singlet under hypercubic rotations (corresponding to summing over $\mu$ ), and a component which is part of a 3-d hypercubic irrep (the diagonal part of the two-index symmetric tensor representation of the Euclidean rotation group). The former is not of interest here, since the sum over $\mu$ decouples the spin and taste indices. It is the latter which we need to match onto mesonic operators.

It is straightforward to do this matching. For each of the operators in Tables III and IV we need to replace " $\partial_{\mu} \partial_{\mu}$ " with " $\partial_{\mu} \partial_{\mu}-(1 / 4) \sum_{\rho} \partial_{\rho} \partial_{\rho}$ ". This projects out the singlet component. The singlet component has decoupled spin and taste indices, however, so we can drop it if we wish, and keep only " $\partial_{\mu} \partial_{\mu}$ ". Doing so gives the following simple prescription: keep the operators in Tables [II and IV] as is, but substitute $F_{R, L} \longrightarrow F(\mu)_{R, L}$. In this way the spin and taste indices become correlated. Now that the operator mapping is done for fixed $\mu$, we can set $F(\mu)$ to the appropriate taste matrix (in this case $\xi_{\mu \nu}$ ) and then sum over the remaining indices (in this case both $\mu$ and $\nu$ with the constraint that $\mu \neq \nu)$. For example, after completing the three steps of the mapping procedure outlined above for operator 1 (Table III) we obtain

$$
\sum_{\mu} \sum_{\nu \neq \mu} \operatorname{Str}\left(\partial_{\mu} \Sigma^{\dagger} \partial_{\mu} \Sigma \Sigma^{\dagger} \xi_{\mu \nu} \Sigma \xi_{\nu \mu}\right)
$$

This operator, like the $S_{6}^{F F(B)}$ operator it came from, breaks the symmetries down to the lattice spin-taste group $\Gamma_{4} \rtimes S W_{4}$.

Carrying out the same procedure for all the types in Tables [II and IV] leads to the mesonic operators collected in the upper panel of Table $\mathrm{XIX}$. Note that the mapping of the four-fermion operator $\left[A_{\mu} \times T_{\mu}\right]$ leads to the same set of mesonic operators as those from $\left[V_{\mu} \times T_{\mu}\right]$.

The mapping of operators $\left[T_{\mu} \times V_{\mu}\right]$ and $\left[T_{\mu} \times A_{\mu}\right]$ can be done similarly. The chiral structure is given by

$$
\mathcal{O}^{\prime \prime}(\mu)_{F}=\sum_{\nu \neq \mu} \overline{Q_{L}}\left(\gamma_{\mu \nu} \otimes \widetilde{F}(\mu)_{L}\right) Q_{R} \overline{Q_{R}}\left(\gamma_{\nu \mu} \otimes \widetilde{F}(\mu)_{R}\right) Q_{L}
$$

where the taste spurions depend on $\mu$, and $\mu$ is not (yet) summed. ${ }^{11,12}$ On the other hand, $\nu$ is summed, so the rotational properties of the operator are the same as those of $\mathcal{O}(\mu)_{F}$ discussed above. Thus when it is mapped onto mesonic operators with two derivatives their indices can both be set to $\mu$. The types of mesonic operators that arise are as for $[S \times V, A]$, except that here we need keep only those with one $\widetilde{F}_{L}$ and one $\widetilde{F}_{R}$, namely operator types 35 -42 in Table VII Furthermore, here we must substitute $\widetilde{F}_{R, L} \longrightarrow \widetilde{F}(\mu)_{R, L}$ (with $F(\mu)_{R, L} \rightarrow \xi_{\mu}$ or $\xi_{\mu 5}$ in the end). The resulting specific mesonic operators are collected in the lower panel of Table XIX

In Ref. [12] it was claimed that one of the operators generated by $\left[T_{\mu} \times V_{\mu}\right]$ at $\mathcal{O}\left(a^{2} p^{2}\right)$ was

$$
\sum_{\mu \neq \nu \neq \rho \neq \mu} \operatorname{Str}\left[\left(\partial_{\mu}-\partial_{\nu}\right) \Sigma \xi_{\rho}\right] \operatorname{Str}\left[\left(\partial_{\mu}-\partial_{\nu}\right) \Sigma \xi_{\rho}\right]
$$

This operator does not, however, appear in our lists. In fact, it is not invariant under the "spin-taste locked" rotation

$$
\begin{array}{ll}
\partial_{\nu} \rightarrow \partial_{\mu}, & \partial_{\mu} \rightarrow-\partial_{\nu} \\
\gamma_{\nu} \rightarrow \gamma_{\mu}, & \gamma_{\mu} \rightarrow-\gamma_{\nu} \\
\xi_{\nu} \rightarrow \xi_{\mu}, & \xi_{\mu} \rightarrow-\xi_{\nu} .
\end{array}
$$

which corresponds to a lattice $S W_{4}$ transformation [12, 23]. Thus it does not arise.

\footnotetext{
11 Note that the chiral structure of $\left[T_{\mu} \times V_{\mu}\right]$ is not the same as that of $[T \times V]$.

12 We thank Claude Bernard for pointing out an error in our discussion of this operator in earlier version of this paper.
} 


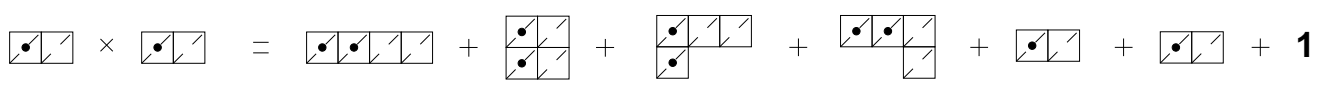

FIG. 6: The product of two adjoints in $S U(4 N \mid 4 M)$. The result also applies to $S U(N)$ for $N>3$ (with the dashed lines removed).

\section{Double insertions of four-fermion operators}

Mesonic operators of $\mathcal{O}\left(a^{4}\right)$ can arise from the combination of two four-fermion operators, each of which brings a power of $a^{2}$, or by mapping a single $\mathcal{O}\left(a^{4}\right)$ quark-level operator. However, as noted in Sec. II the $\mathcal{O}\left(a^{4}\right)$ operators do not break any additional symmetries and do not contain any more correlated taste indices, so they do not lead to additional mesonic operators.

Thus we consider only the mesonic operators which result from two insertions of four-fermion operators. These two insertions need not have same spin or taste, and may come from either $S_{6}^{F F(A)}$ or $S_{6}^{F F(B)}$. We first consider pairs of $F F(A)$ operators, separating the various possible spin combinations. We then generalize these results to the case in which one or both of the operators is in $S_{6}^{F F(B)}$.

a. Spin $V$ or $A$ four-fermion operators with spin $V$ or $A$ four-fermion operators

Operators of $\mathcal{O}\left(a^{4}\right)$ must contain four taste spurions - two from each four-fermion operator. The taste spurions corresponding to operators with spins $V$ and $A$ are $F_{L}$ and $F_{R}$, which in the end can be set to $S$, $P$ or $T$. In fact, as we have seen above, the taste singlet always leads to operators which either vanish or are of the same form as LO operators. We have checked that this remains true for $\mathcal{O}\left(a^{4}\right)$ operators, and so drop taste $S$ from the beginning. Thus we can set $\operatorname{Str}\left(F_{L}\right)=\operatorname{Str}\left(F_{R}\right)=0$, which simplifies the operator enumeration.

There are five parity-invariant ways to combine the four taste spurions:

$$
\begin{aligned}
& \left(F_{L} F_{R}\right)\left(F_{L}^{\prime} F_{R}^{\prime}\right) \\
& \left(F_{L} F_{L}\right)\left(F_{L}^{\prime} F_{R}^{\prime}\right)+p . c . \\
& \left(F_{L} F_{R}\right)\left(F_{L}^{\prime} F_{L}^{\prime}\right)+p . c . \\
& \left(F_{L} F_{L}\right)\left(F_{L}^{\prime} F_{L}^{\prime}\right)+p . c . \\
& \left(F_{L} F_{L}\right)\left(F_{R}^{\prime} F_{R}^{\prime}\right)+p . c .
\end{aligned}
$$

Here we use primes to distinguish between spurions from the separate four-fermion operators because they may have different tastes. To determine the number of linearly-independent chiral operators, we use graded group theory as before. For simplicity, we build operators out of the left-handed basis defined in (A7). Both $F_{L}$ and $\Sigma F_{R} \Sigma^{\dagger}$ are now supertraceless, so they have no singlet component and transform as adjoints under the chiral symmetry group. The corresponding $\mathcal{O}\left(a^{4}\right)$ operators are therefore chiral singlets contained in the product of four adjoints, with various symmetries imposed based on the handedness of the four spurions.

Operators of the first type, $\left(F_{L} F_{R}\right)\left(F_{L}^{\prime} F_{R}^{\prime}\right)$, contain four different taste spurions, and therefore possess no additional symmetry. The number of linearly-independent operators of this sort is simply the number of singlets in the product of four adjoints. (The product of two adjoints is shown in Fig. 6]) There are nine such operators, and they are given in Table IX Most, however, reduce to LO operators or field-independent constants after setting $F$ and $F^{\prime}$ to specific taste matrices; only three are new.

Operators of the last type, $\left(F_{L} F_{L}\right)\left(F_{R}^{\prime} F_{R}^{\prime}\right)+p . c .$, contain two pairs of identical taste spurions. They therefore come from the singlet irreps in the product of one symmetric product of two adjoints with another symmetric product of two adjoints, i.e. the product of Fig. 5 with itself. There are four such singlets; they correspond to the operators in Table $\mathrm{X}$ However, only two of these operators are new and nontrivial.

It is straightforward to show that the other three spurion combinations in (A26) do not generate any additional $\mathcal{O}\left(a^{4}\right)$ operators, so we choose to omit their discussion here. All that remains is the somewhat tedious task of replacing the taste spurions with specific taste matrices. There are three non-trivial pairings of tastes, leading to three different taste matrix substitutions:

$$
\begin{aligned}
& {[V, A \times P] \text { with }[V, A \times P] \Rightarrow F=F^{\prime}=\xi_{5}} \\
& {[V, A \times P] \text { with }[V, A \times T] \Rightarrow F=\xi_{5}, \quad F^{\prime}=\xi_{\mu \nu}} \\
& {[V, A \times T] \text { with }[V, A \times T] \Rightarrow F=\xi_{\mu \nu}, \quad F^{\prime}=\xi_{\rho \sigma} .}
\end{aligned}
$$




\begin{tabular}{lc}
\hline \hline \multicolumn{1}{c}{ Operator } & Keep? \\
\hline 69. $\operatorname{Str}\left(F_{L} \Sigma F_{R} \Sigma^{\dagger} F_{L}^{\prime} \Sigma F_{R}^{\prime} \Sigma^{\dagger}\right)$ & Yes - combined with 74 \\
70. $\operatorname{Str}\left(F_{L} \Sigma F_{R} F_{R}^{\prime} \Sigma^{\dagger} F_{L}^{\prime}\right)$ & No \\
71. $\operatorname{Str}\left(F_{L} F_{L}^{\prime} \Sigma F_{R} F_{R}^{\prime} \Sigma^{\dagger}\right)$ & No \\
72. $\operatorname{Str}\left(F_{L} F_{L}^{\prime} \Sigma F_{R}^{\prime} F_{R} \Sigma^{\dagger}\right)$ & No \\
73. $\operatorname{Str}\left(F_{L} \Sigma F_{R}^{\prime} F_{R} \Sigma^{\dagger} F_{L}^{\prime}\right)$ & No \\
74. $\operatorname{Str}\left(F_{L} \Sigma F_{R}^{\prime} \Sigma^{\dagger} F_{L}^{\prime} \Sigma F_{R} \Sigma^{\dagger}\right)$ & Yes - combined with 69 \\
75. $\operatorname{Str}\left(F_{L} \Sigma F_{R} \Sigma^{\dagger}\right) \operatorname{Str}\left(F_{L}^{\prime} \Sigma F_{R}^{\prime} \Sigma^{\dagger}\right)$ & Yes \\
76. $\operatorname{Str}\left(F_{L} F_{L}^{\prime}\right) \operatorname{Str}\left(F_{R} F_{R}^{\prime}\right)$ & No \\
77. $\operatorname{Str}\left(F_{L} \Sigma F_{R}^{\prime} \Sigma^{\dagger}\right) \operatorname{Str}\left(F_{L}^{\prime} \Sigma F_{R} \Sigma^{\dagger}\right)$ & Yes \\
\hline \hline
\end{tabular}

TABLE IX: The nine linearly-independent $\mathcal{O}\left(a^{4}\right)$ operators composed of taste spurions $F_{L}, F_{R}, F_{L}^{\prime}$ and $F_{R}^{\prime}$.

\begin{tabular}{lc}
\hline \hline \multicolumn{1}{c}{ Operator } & Keep? \\
\hline 78. $\operatorname{Str}\left(F_{L} F_{L} \Sigma F_{R}^{\prime} F_{R}^{\prime} \Sigma^{\dagger}\right)+$ p.c. & No \\
79. $\operatorname{Str}\left(F_{L} \Sigma F_{R}^{\prime} \Sigma^{\dagger} F_{L} \Sigma F_{R}^{\prime} \Sigma^{\dagger}\right)+$ p.c. & Yes \\
80. $\operatorname{Str}\left(F_{L} F_{L}\right) \operatorname{Str}\left(F_{R}^{\prime} F_{R}^{\prime}\right)+$ p.c. & No \\
81. $\operatorname{Str}\left(F_{L} \Sigma F_{R}^{\prime} \Sigma^{\dagger}\right) \operatorname{Str}\left(F_{L} \Sigma F_{R}^{\prime} \Sigma^{\dagger}\right)+$ p.c. & Yes \\
\hline \hline
\end{tabular}

TABLE X: The four linearly-independent $\mathcal{O}\left(a^{4}\right)$ operators composed of two $F_{L}$ and two $F_{R}^{\prime}$ taste spurions.

Note that, in the case of taste $T$ with $T$, the two pairs of taste indices must be contracted separately because $S_{6}^{F F(A)}$ respects both $S O(4)$-taste and Lorentz symmetry. The three corresponding sets of chiral operators are given in Table XX

\section{b. Spin $S, P$, or $T$ four-fermion operators with spin $S, P$, or $T$ four-fermion operators}

The taste spurions corresponding to operators with spins $S, P$, and $T$ are $\widetilde{F_{L}}$ and $\widetilde{F_{R}}$, and can be combined in five parity-invariant ways:

$$
\begin{aligned}
& \left(\widetilde{F_{L}} \widetilde{F_{R}}\right)\left({\widetilde{F_{L}}}^{\prime}{\widetilde{F_{R}}}^{\prime}\right) \\
& \left(\widetilde{F_{L}}{\widetilde{F_{L}}}^{\prime}\right)\left({\widetilde{F_{L}}}^{\prime}{\widetilde{F_{R}}}^{\prime}\right)+p . c . \\
& \left(\widetilde{F_{L}} \widetilde{F_{R}}\right)\left({\widetilde{F_{L}}}^{\prime}{\widetilde{F_{L}}}^{\prime}\right)+p . c . \\
& \left(\widetilde{F_{L}} \widetilde{F_{L}}\right)\left(\widetilde{F_{L}} \widetilde{F}^{\prime}\right)+p . c . \\
& \left(\widetilde{F_{L}} \widetilde{F_{L}}\right)\left({\widetilde{F_{R}}}^{\prime}{\widetilde{F_{R}}}^{\prime}\right)+\text { p.c. }
\end{aligned}
$$

In this case we build operators out of the left-handed basis defined in (A12). Neither $F_{L} \Sigma^{\dagger}$ nor $\Sigma F_{R}$ is supertraceless, so each transforms as a bifundamental under the chiral symmetry group. Thus their product contains slightly more singlet irreps, and therefore generates more operators, than in the spin $V$ case.

Operators of type $\left(\widetilde{F_{L}} \widetilde{F_{R}}\right)\left({\widetilde{F_{L}}}^{\prime}{\widetilde{F_{R}}}^{\prime}\right)$ correspond to singlet irreps in the product of four bifundamentals, i.e. the product of Fig. [3 with itself. There are twenty-four singlet irreps in this product, but we only show, in Table XI the three that lead to new $\mathcal{O}\left(a^{4}\right)$ operators.

In operators of type $\left(\left(\widetilde{F_{L}} \widetilde{F_{L}}\right)\left({\widetilde{F_{L}}}^{\prime}{\widetilde{F_{R}}}^{\prime}\right)+\right.$ p.c. $)$ and $\left(\left(\widetilde{F_{L}} \widetilde{F_{R}}\right)\left({\widetilde{F_{L}}}^{\prime}{\widetilde{F_{L}}}^{\prime}\right)+\right.$ p.c. $)$, one pair of taste spurions is identical. Thus the number of such operators equals the number of singlet irreps in the product of one symmetric product of two bifundamentals with another two bifundamentals, i.e. the product of Fig. 目 with Fig. 3] This product contains fourteen singlets, so there are twenty-eight such operators. However, only eight are new, and these are listed in Table XII

Finally, in operators of type $\left(\left(\widetilde{F_{L}} \widetilde{F_{L}}\right)\left({\widetilde{F_{L}}}^{\prime}{\widetilde{F_{L}}}^{\prime}\right)+\right.$ p.c. $)$ and $\left(\left(\widetilde{F_{L}} \widetilde{F_{L}}\right)\left({\widetilde{F_{R}}}^{\prime}{\widetilde{F_{R}}}^{\prime}\right)+\right.$ p.c. $)$, both pairs of taste spurions are identical. Therefore these operators correspond to chiral singlets in the product of one symmetric product of 


\begin{tabular}{|c|c|}
\hline Operator & Keep? \\
\hline 82. $\operatorname{Str}\left(\widetilde{F_{L}} \Sigma^{\dagger} \widetilde{F_{L}} \Sigma^{\dagger}\right) \operatorname{Str}\left(\Sigma \widetilde{F_{R}} \Sigma{\widetilde{F_{R}}}^{\prime}\right)$ & Yes \\
\hline 83. $\operatorname{Str}\left(\widetilde{F_{L}} \Sigma^{\dagger}{\widetilde{F_{L}}}^{\prime} \Sigma^{\dagger}\right) \operatorname{Str}\left(\Sigma \widetilde{F_{R}}\right) \operatorname{Str}\left(\Sigma{\widetilde{F_{R}}}^{\prime}\right)+$ p.c. & Yes \\
\hline 84. $\operatorname{Str}\left(\widetilde{F}_{L} \Sigma^{\dagger}\right) \operatorname{Str}\left(\widetilde{F}_{L}^{\prime} \Sigma^{\dagger}\right) \operatorname{Str}\left(\Sigma \widetilde{F}_{R}\right) \operatorname{Str}\left(\Sigma \widetilde{F}_{R}^{\prime}\right)$ & Yes \\
\hline
\end{tabular}

TABLE XI: The three non-trivial $\mathcal{O}\left(a^{4}\right)$ operators composed of the four taste spurions $\widetilde{F}_{L}, \widetilde{F}_{R}, \widetilde{F}_{L}^{\prime}$ and $\widetilde{F}_{R}^{\prime}$.

\begin{tabular}{lc}
\hline \hline Operator & Keep? \\
\hline 85. $\operatorname{Str}\left(\widetilde{F}_{L} \Sigma^{\dagger} \widetilde{F}_{L} \Sigma^{\dagger} \widetilde{F}_{L}^{\prime} \Sigma^{\dagger}\right) \operatorname{Str}\left(\Sigma \widetilde{F}_{R}^{\prime}\right)+$ p.c. & Yes \\
86. $\operatorname{Str}\left(\widetilde{F}_{L}^{\prime} \Sigma^{\dagger} \widetilde{F}_{L}^{\prime} \Sigma^{\dagger} \widetilde{F}_{L} \Sigma^{\dagger}\right) \operatorname{Str}\left(\Sigma \widetilde{F}_{R}\right)+$ p.c. & Yes \\
87. $\operatorname{Str}\left(\widetilde{F}_{L} \Sigma^{\dagger} \widetilde{F}_{L} \Sigma^{\dagger}\right) \operatorname{Str}\left(\widetilde{F}_{L}^{\prime} \Sigma^{\dagger}\right) \operatorname{Str}\left(\Sigma \widetilde{F}_{R}^{\prime}\right)+$ p.c. & Yes \\
88. $\operatorname{Str}\left(\widetilde{F}_{L}^{\prime} \Sigma^{\dagger} \widetilde{F}_{L}^{\prime} \Sigma^{\dagger}\right) \operatorname{Str}\left(\widetilde{F}_{L} \Sigma^{\dagger}\right) \operatorname{Str}\left(\Sigma \widetilde{F}_{R}\right)+$ p.c. & Yes \\
89. $\operatorname{Str}\left(\widetilde{F}_{L} \Sigma^{\dagger} \widetilde{F}_{L}^{\prime} \Sigma^{\dagger}\right) \operatorname{Str}\left(\widetilde{F}_{L} \Sigma^{\dagger}\right) \operatorname{Str}\left(\Sigma \widetilde{F}_{R}^{\prime}\right)+$ p.c. & Yes \\
90. $\operatorname{Str}\left(\widetilde{F}_{L}^{\prime} \Sigma^{\dagger} \widetilde{F}_{L} \Sigma^{\dagger}\right) \operatorname{Str}\left(\widetilde{F}_{L}^{\prime} \Sigma^{\dagger}\right) \operatorname{Str}\left(\Sigma \widetilde{F}_{R}\right)+$ p.c. & Yes \\
91. $\operatorname{Str}\left(\widetilde{F}_{L} \Sigma^{\dagger}\right) \operatorname{Str}\left(\widetilde{F}_{L} \Sigma^{\dagger}\right) \operatorname{Str}\left(\widetilde{F}_{L}^{\prime} \Sigma^{\dagger}\right) \operatorname{Str}\left(\Sigma \widetilde{F}_{R}^{\prime}\right)+$ p.c. & Yes \\
92. $\operatorname{Str}\left(\widetilde{F}_{L}^{\prime} \Sigma^{\dagger}\right) \operatorname{Str}\left(\widetilde{F}_{L}^{\prime} \Sigma^{\dagger}\right) \operatorname{Str}\left(\widetilde{F}_{L} \Sigma^{\dagger}\right) \operatorname{Str}\left(\Sigma \widetilde{F}_{R}\right)+$ p.c. & Yes \\
\hline \hline
\end{tabular}

TABLE XII: The eight non-trivial $\mathcal{O}\left(a^{4}\right)$ operators composed of two $\widetilde{F}_{L}$, one $\widetilde{F}_{L}^{\prime}$ and one $\widetilde{F}_{R}^{\prime}$ taste spurions.

bifundamentals with another symmetric product of bifundamentals, or of Fig. 4 with itself. There are ten such singlets, and therefore twenty such operators, but only fourteen are new. We list these in Table XIII.

As we know from the previous section, spin $T$ four-fermion operators generate a subset of those chiral operators generated by spins $S$ and $P$; we can therefore ignore them. Thus, when pairing four-fermion operators, there are three combinations requiring different taste matrix substitutions:

$$
\begin{aligned}
& {[S, P \times V] \text { with }[S, P \times V] \Rightarrow \widetilde{F}=\xi_{\mu}, \quad \widetilde{F}^{\prime}=\xi_{\nu}} \\
& {[S, P \times V] \text { with }[S, P \times A] \Rightarrow \widetilde{F}=\xi_{\mu}, \quad \widetilde{F}^{\prime}=\xi_{\nu 5}} \\
& {[S, P \times A] \text { with }[S, P \times A] \Rightarrow \widetilde{F}=\xi_{\mu 5}, \quad \widetilde{F}^{\prime}=\xi_{\nu 5} .}
\end{aligned}
$$

In all of these operators $\mu$ and $\nu$ must be separately contracted to maintain $S O(4)$-taste and Lorentz symmetry.

\begin{tabular}{llc}
\hline \hline & \multicolumn{1}{c}{ Operator } & Keep? \\
\hline 93. & $\operatorname{Str}\left(\widetilde{F}_{L} \Sigma^{\dagger} \widetilde{F}_{L} \Sigma^{\dagger} \widetilde{F}_{L}^{\prime} \Sigma^{\dagger} \widetilde{F}_{L}^{\prime} \Sigma^{\dagger}\right)+$ p.c. & Yes \\
94. & $\operatorname{Str}\left(\widetilde{F}_{L} \Sigma^{\dagger} \widetilde{F}_{L}^{\prime} \Sigma^{\dagger} \widetilde{F}_{L} \Sigma^{\dagger} \widetilde{F}_{L}^{\prime} \Sigma^{\dagger}\right)+$ p.c. & Yes \\
95. & $\operatorname{Str}\left(\widetilde{F}_{L} \Sigma^{\dagger} \widetilde{F}_{L} \Sigma^{\dagger} \widetilde{F}_{L}^{\prime} \Sigma^{\dagger}\right) \operatorname{Str}\left(\widetilde{F}_{L}^{\prime} \Sigma^{\dagger}\right)+$ p.c. & Yes \\
96. & $\operatorname{Str}\left(\widetilde{F}_{L}^{\prime} \Sigma^{\dagger} \widetilde{F}_{L}^{\prime} \Sigma^{\dagger} \widetilde{F}_{L} \Sigma^{\dagger}\right) \operatorname{Str}\left(\widetilde{F}_{L} \Sigma^{\dagger}\right)+$ p.c. & Yes \\
97. & $\operatorname{Str}\left(\widetilde{F}_{L} \Sigma^{\dagger} \widetilde{F}_{L} \Sigma^{\dagger}\right) \operatorname{Str}\left(\widetilde{F}_{L}^{\prime} \Sigma^{\dagger} \widetilde{F}_{L}^{\prime} \Sigma^{\dagger}\right)+$ p.c. & Yes \\
98. & $\operatorname{Str}\left(\widetilde{F}_{L} \Sigma^{\dagger} \widetilde{F}_{L}^{\prime} \Sigma^{\dagger}\right) \operatorname{Str}\left(\widetilde{F}_{L} \Sigma^{\dagger} \widetilde{F}_{L}^{\prime} \Sigma^{\dagger}\right)+$ p.c. & Yes \\
99. & $\operatorname{Str}\left(\widetilde{F}_{L} \Sigma^{\dagger} \widetilde{F}_{L} \Sigma^{\dagger}\right) \operatorname{Str}\left(\widetilde{F}_{L}^{\prime} \Sigma^{\dagger}\right) \operatorname{Str}\left(\widetilde{F}_{L}^{\prime} \Sigma^{\dagger}\right)+$ p.c. & Yes \\
100. & $\operatorname{Str}\left(\widetilde{F}_{L} \Sigma^{\dagger} \widetilde{F}_{L}^{\prime} \Sigma^{\dagger}\right) \operatorname{Str}\left(\widetilde{F}_{L} \Sigma^{\dagger}\right) \operatorname{Str}\left(\widetilde{F}_{L}^{\prime} \Sigma^{\dagger}\right)+p . c$. & Yes \\
101. & $\operatorname{Str}\left(\widetilde{F}_{L}^{\prime} \Sigma^{\dagger} \widetilde{F}_{L}^{\prime} \Sigma^{\dagger}\right) \operatorname{Str}\left(\widetilde{F}_{L} \Sigma^{\dagger}\right) \operatorname{Str}\left(\widetilde{F}_{L} \Sigma^{\dagger}\right)+$ p.c. & Yes \\
102. & $\operatorname{Str}\left(\widetilde{F}_{L} \Sigma^{\dagger}\right) \operatorname{Str}\left(\widetilde{F}_{L} \Sigma^{\dagger}\right) \operatorname{Str}\left(\widetilde{F}_{L}^{\prime} \Sigma^{\dagger}\right) \operatorname{Str}\left(\widetilde{F}_{L}^{\prime} \Sigma^{\dagger}\right)+$ p.c.. & Yes \\
103. & $\operatorname{Str}\left(\widetilde{F}_{L} \Sigma^{\dagger} \widetilde{F}_{L} \Sigma^{\dagger}\right) \operatorname{Str}\left(\Sigma \widetilde{F}_{R}^{\prime} \Sigma \widetilde{F}_{R}^{\prime}\right)+p . c$. & Yes \\
104. $\operatorname{Str}\left(\widetilde{F}_{L} \Sigma^{\dagger} \widetilde{F}_{L} \Sigma^{\dagger}\right) \operatorname{Str}\left(\Sigma \widetilde{F}_{R}^{\prime}\right) \operatorname{Str}\left(\Sigma \widetilde{F}_{R}^{\prime}\right)+$ p.c. & Yes \\
105. $\operatorname{Str}\left(\widetilde{F}_{L} \Sigma^{\dagger}\right) \operatorname{Str}\left(\widetilde{F}_{L} \Sigma^{\dagger}\right) \operatorname{Str}\left(\Sigma \widetilde{F}_{R}^{\prime} \Sigma \widetilde{F}_{R}^{\prime}\right)+$ p.c. & Yes \\
106. $\operatorname{Str}\left(\widetilde{F}_{L} \Sigma^{\dagger}\right) \operatorname{Str}\left(\widetilde{F}_{L} \Sigma^{\dagger}\right) \operatorname{Str}\left(\Sigma \widetilde{F}_{R}^{\prime}\right) \operatorname{Str}\left(\Sigma \widetilde{F}_{R}^{\prime}\right)+$ p.c. & Yes \\
\hline \hline & &
\end{tabular}

TABLE XIII: The fourteen non-trivial $\mathcal{O}\left(a^{4}\right)$ operators composed of two $\widetilde{F}_{L}$ and either two $\widetilde{F}_{L}^{\prime}$ or two $\widetilde{F}_{R}^{\prime}$ taste spurions. 


\begin{tabular}{lc}
\hline \hline \multicolumn{1}{c}{ Operator } & Keep? \\
\hline 107. $\operatorname{Str}\left(F_{L} \Sigma \widetilde{F_{R}} \Sigma F_{R} \Sigma^{\dagger} \widetilde{F_{L}} \Sigma^{\dagger}\right)$ & Yes \\
108. $\operatorname{Str}\left(F_{L} \Sigma \widetilde{F_{R}} \Sigma F_{R} \Sigma^{\dagger}\right) \operatorname{Str}\left(\widetilde{F_{L}} \Sigma^{\dagger}\right)$ & Yes - combined with 109 \\
109. $\operatorname{Str}\left(F_{L} \Sigma F_{R} \Sigma^{\dagger} \widetilde{F_{L}} \Sigma^{\dagger}\right) \operatorname{Str}\left(\Sigma \widetilde{F}_{R}\right)$ & Yes - combined with 108 \\
110. $\operatorname{Str}\left(F_{L} \Sigma F_{R} \Sigma^{\dagger}\right) \operatorname{Str}\left(\widetilde{F_{L}} \Sigma^{\dagger}\right) \operatorname{Str}\left(\Sigma \widetilde{F_{R}}\right)$ & Yes \\
111. $\operatorname{Str}\left(\widetilde{F_{L}} \Sigma^{\dagger} \widetilde{F_{L}} \Sigma^{\dagger} F_{L} \Sigma F_{R} \Sigma^{\dagger}\right)+$ p.c. & Yes \\
112. $\operatorname{Str}\left(\widetilde{F_{L}} \Sigma^{\dagger} \widetilde{F_{L}} \Sigma^{\dagger}\right) \operatorname{Str}\left(F_{L} \Sigma F_{R} \Sigma^{\dagger}\right)+$ p.c. & Yes \\
113. $\operatorname{Str}\left(\widetilde{F_{L}} \Sigma^{\dagger} F_{L} \Sigma F_{R} \Sigma^{\dagger}\right) \operatorname{Str}\left(\widetilde{F_{L}} \Sigma^{\dagger}\right)+$ p.c. & Yes \\
114. $\operatorname{Str}\left(\widetilde{F_{L}} \Sigma^{\dagger}\right) \operatorname{Str}\left(\widetilde{F_{L}} \Sigma^{\dagger}\right) \operatorname{Str}\left(F_{L} \Sigma F_{R} \Sigma^{\dagger}\right)+$ p.c. & Yes \\
\hline \hline
\end{tabular}

TABLE XIV: The eight non-trivial $\mathcal{O}\left(a^{4}\right)$ operators composed of spurions $F_{L}, F_{R}$ and two $\widetilde{F}^{\prime} s$.

Table XXI contains all of the corresponding operators in the chiral Lagrangian.

c. Spin $V$ or A four-fermion operators with spin $S, P$, or $T$ four-fermion operators

Operators that arise from the insertion of one operator with spin $V$ or $A$ and one with spin $S, P$, or $T$ necessarily have mixed tastes. Each four-fermion operator contributes two taste spurions, so the corresponding chiral operators contain two $F$ 's and two $\widetilde{F}$ 's. The five parity-invariant ways of combining these spurions are

$$
\begin{aligned}
& \left(F_{L} F_{R}\right)\left(\widetilde{F_{L}} \widetilde{F_{R}}\right) \\
& \left(F_{L} F_{L}\right)\left(\widetilde{F_{L}} \widetilde{F_{R}}\right)+p . c . \\
& \left(F_{L} F_{R}\right)\left(\widetilde{F_{L}} \widetilde{F_{L}}\right)+p . c . \\
& \left(F_{L} F_{L}\right)\left(\widetilde{F_{L}} \widetilde{F_{L}}\right)+p . c . \\
& \left(F_{L} F_{L}\right)\left(\widetilde{F_{R}} \widetilde{F_{R}}\right)+p . c .
\end{aligned}
$$

We do not need to use primes, since the spurions from the two four-fermion operators are already distinguished by the presence or absence of the tilde. As before, we build our operators using a left-handed basis, and in this case we must use both taste spurion bases defined in (A7) and (A12). Recall that under chiral symmetry transformations, $F_{L}$ and $\Sigma F_{R} \Sigma^{\dagger}$ transform as adjoints, while $\widetilde{F_{L}} \Sigma^{\dagger}$ and $\Sigma \widetilde{F_{R}}$ transform as bifundamentals. Thus the group theory counting will be slightly different than in the two previous sections, and each of the five spurion combinations in A30 must be considered separately.

First consider operators of type $\left(F_{L} F_{R}\right)\left(\widetilde{F_{L}} \widetilde{F_{R}}\right)$. These come from the singlet irreps in the product of two adjoints with two bifundamentals. There are fourteen, but only four correspond to new NLO operators. Next consider the third type, $\left(F_{L} F_{R}\right)\left(\widetilde{F_{L}} \widetilde{F_{L}}\right)+$ p.c.. Because there are two $\widetilde{F_{L}}$ 's, chiral operators correspond to chiral singlets in the product of two adjoints with the symmetric product of two bifundamentals. This product contains eight singlets, but only leads to four new NLO operators. Finally, the other three combinations in A30 can be shown not to produce any new operators, so we do not discuss them further. Table XIV shows the eight new operators that can be built out of two $F$ 's and two $\widetilde{F}$ 's.

In this mixed-taste and mixed-spurion case, there are four possible four-fermion operator combinations and corresponding taste matrix substitutions:

$$
\begin{aligned}
& {[V, A \times P] \text { with }[S, P \times V] \Rightarrow F=\xi_{5}, \quad \widetilde{F}=\xi_{\mu}} \\
& {[V, A \times P] \text { with }[S, P \times A] \Rightarrow F=\xi_{5}, \quad \widetilde{F}=\xi_{\mu 5}} \\
& {[V, A \times T] \text { with }[S, P \times V] \Rightarrow F=\xi_{\mu \nu}, \quad \widetilde{F}=\xi_{\rho}} \\
& {[V, A \times T] \text { with }[S, P \times A] \Rightarrow F=\xi_{\mu \nu}, \quad \widetilde{F}=\xi_{\rho 5} .}
\end{aligned}
$$

Once again, all index pairs are contracted separately, thereby maintaining $S O(4)$-taste and Lorentz symmetry. The chiral operators of $\mathcal{O}\left(a^{4}\right)$ arising from the insertion of a spin $V$ or $A$ four-fermion operator and the insertion of a spin $S, P$, or $T$ four-fermion operator are given in Table XXII 
d. Double insertions involving four-fermion operators in $S_{6}^{F F(B)}$

In this section we determine mesonic operators of $\mathcal{O}\left(a^{4}\right)$ which arise from the double insertion of four-fermion operators, at least one of which is from $S_{6}^{F F(B)}$. As in the single four-fermion operator insertion case, the chiral structures are identical to those for insertions of the corresponding operators in $S_{6}^{F F(A)}$, but new index structures arise which break the $S O(4)$ taste symmetry. Also like before, we are not interested in operators which do not break this symmetry, since these have already been completely determined. Thus we first consider insertions of two four-fermion operators from $S_{6}^{F F(B)}$. After this discussion, it will be easy to see that combinations of one $F F(A)$ operator with one $F F(B)$ operator cannot generate anything additional, and in fact only generate operators that respect $S O(4)$-taste and Lorentz symmetries.

Although the spin order is reversed as compared to previous sections, we begin with double insertions of $\left[T_{\mu} \times V_{\mu}, A_{\mu}\right]$. This is because it is easy to guess the correct $S O(4)$-taste symmetry breaking chiral operators. They have the same chiral structure as a subset of operators that come from double insertions of $[S, P \times V, A]$, namely those which arise from $L-R$ cross-terms (operators 82-84). A quick glance at Table XXI reveals that there is only one possible symmetrybreaking index contraction: $\mu=\nu$. As this contraction does not break any of the discrete lattice symmetries, it must be present at some order in our power counting. We can show, however, that it appears at $\mathcal{O}\left(a^{4}\right)$ using an argument similar to those in Section A3 For fixed $\mu$ and $\mu^{\prime}$, the general spin-taste index structure of these operators (ignoring factors of $\xi_{5}$ in $A_{\mu}$ ) is the following:

$$
\sum_{\nu \neq \mu} \sum_{\nu^{\prime} \neq \mu^{\prime}}(\mu \nu \otimes \mu)(\nu \mu \otimes \mu)\left(\mu^{\prime} \nu^{\prime} \otimes \mu^{\prime}\right)\left(\nu^{\prime} \mu^{\prime} \otimes \mu^{\prime}\right)
$$

Because mesonic operators of $\mathcal{O}\left(a^{4}\right)$ have no derivatives, invariance under the lattice symmetries implies that they must be singlets under hypercubic rotations. As was discussed previously, both $\mu \mu$ and $\mu^{\prime} \mu^{\prime}$ contain a singlet and a 3 -d irrep. Thus their product contains two overall singlets - one from the product of the singlets and one from the product of the 3-d irreps. Only the latter gives new operators, for it contains a part in which $\mu=\mu^{\prime}$, confirming our initial guess. The $S O(4)$-taste symmetry breaking operators arising from double insertions of $\left[T_{\mu} \times V_{\mu}, A_{\mu}\right]$ are given in the first two panels of Table XXIII

Next we proceed to mixed-spin operators, i.e. the result of an insertion of $\left[V_{\mu}, A_{\mu} \times T_{\mu}\right]$ with an insertion of $\left[T_{\mu} \times V_{\mu}, A_{\mu}\right]$. These operators have identical chiral structures to the ones in the top portion of Table XXII ${ }^{13}$ Once again, there is only one possible $S O(4)$-taste breaking index contraction, $\mu=\rho$, but we must still show that this is the correct mapping of the four-fermion operator product. ${ }^{14}$ In this case, the operators have the following index structure at fixed $\mu$ and $\mu^{\prime}$ :

$$
\sum_{\nu \neq \mu} \sum_{\nu^{\prime} \neq \mu^{\prime}}(\mu \otimes \mu \nu)(\mu \otimes \nu \mu)\left(\mu^{\prime} \nu^{\prime} \otimes \mu^{\prime}\right)\left(\nu^{\prime} \mu^{\prime} \otimes \mu^{\prime}\right)
$$

Just as before, one hypercubic singlet in this product comes from the two 3-d irreps, and contains a part with $\mu=\mu^{\prime}$. This generates the $\mathcal{O}\left(a^{4}\right)$ mixed-spin operators in the third panel of Table XXIII

Finally we address double insertions of $\left[V_{\mu}, A_{\mu} \times T_{\mu}\right]$. This will produce operators with the same chiral structure as double insertions of $[V, A \times T]$. Such operators are given in the bottom portion of Table $\mathrm{XX}$ In this case, multiple combinations of index contractions are possible: $\mu=\rho$ and $\mu=\rho, \nu=\sigma$. Both are allowed by the lattice symmetries, but we must determine whether either arises at $\mathcal{O}\left(a^{4}\right)$. After fixing $\mu$ and $\mu^{\prime}$, the index structure is

$$
\sum_{\nu \neq \mu} \sum_{\nu^{\prime} \neq \mu^{\prime}}(\mu \otimes \mu \nu)(\mu \otimes \nu \mu)\left(\mu^{\prime} \otimes \mu^{\prime} \nu^{\prime}\right)\left(\mu^{\prime} \otimes \nu^{\prime} \mu^{\prime}\right)
$$

Now we can see that the only nontrivial way of producing a hypercubic singlet is $\mu=\mu^{\prime}$, just like in the two previous examples. The indices $\nu$ and $\nu^{\prime}$ are independently summed from the beginning. We conclude that only the first of the two possible index contractions arises from the double insertion of four fermion operators. The final panel of Table XXIII shows the $\mathcal{O}\left(a^{4}\right)$ chiral operators generated by double insertions of $\left[V_{\mu}, A_{\mu} \times T_{\mu}\right]$.

After these examples, it is easy to see that the combination of an $F F(A)$ four-fermion operator with an $F F(B)$ four-fermion operator cannot generate any additional operators. This is because, in the $F F(A)$ operator, spin and

\footnotetext{
13 Note that we do not need the " $-\mu \nu 5$ " parts of $\left[V_{\mu}, A_{\mu} \times T_{\mu}\right]$, since they can be removed by adding an appropriate amount of $[V, A \times T]$, as discussed in Sec. A3 This simplifies the final taste matrix substitution.

14 Note that $\nu=\rho$ does not produce different operators since these are all just dummy indices.
} 
taste indices are completely uncorrelated. Thus these operators contain only a singlet under hypercubic rotations, and can only form a overall singlet by combining with the singlet component of the $F F(B)$ operator. But doing so removes the correlations between taste indices. Such mixed-symmetry double insertions only modify the coefficients of the $\mathcal{O}\left(a^{4}\right) F F(A)$ operators.

A natural question that arises is what happened to the second possible set of index contractions in the $\left[V_{\mu}, A_{\mu} \times T_{\mu}\right]^{2}$ case, in which both $\mu=\mu^{\prime}$ and $\nu=\nu^{\prime}$. This would lead to mesonic operators such as

$$
\sum_{\mu \neq \nu}\left\{\operatorname{Str}\left(\xi_{\mu \nu} \Sigma \xi_{\nu \mu} \Sigma^{\dagger} \xi_{\mu \nu} \Sigma \xi_{\nu \mu} \Sigma^{\dagger}\right)+p . c .\right\}
$$

i.e. with both $\mu$ and $\nu$ appearing four times. Such operators are consistent with all of the lattice symmetries, so they must be present in the chiral Lagrangian at some order. The answer is that these operators are present, but they are of $\mathcal{O}\left(a^{8}\right)$. It is clear from the previous examples that contracting the spin indices to make hypercube singlets can never contract all of the taste indices, some of which are independently summed. Thus chiral operators such as (A35) can only arise if all of the tensor indices are coupled in the quark-level operator itself. This requires a local eight-quark operator, e.g.

$$
\sum_{\mu} \sum_{\nu \neq \mu}\left[\bar{Q}\left(\gamma_{\mu} \otimes \xi_{\mu \nu}\right) Q\right]\left[\bar{Q}\left(\gamma_{\mu} \otimes \xi_{\nu \mu}\right) Q\right]\left[\bar{Q}\left(\gamma_{\mu} \otimes \xi_{\mu \nu}\right) Q\right]\left[\bar{Q}\left(\gamma_{\mu} \otimes \xi_{\nu \mu}\right) Q\right]
$$

which enters only at $\mathcal{O}\left(a^{8}\right)$.

A more physical way of looking at this is in terms of gluon exchange. Taste-changing interactions are due to the exchange of hard gluons with momentum $k \sim \pi / a$. To obtain a complete correlation of taste indices requires a complete correlation of the hard gluon momenta. This can arise directly, for example, with four bilinears connected by four gluons joining at a four-gluon vertex, as in the above operator. The presence of four hard gluon propagators gives the factor $\left(1 / k^{2}\right)^{4} \sim a^{8}$. In contrast, the $\mathcal{O}\left(a^{4}\right)$ mesonic operators that we are considering arise from two separate insertions of $\mathcal{O}\left(a^{2}\right)$ four-fermion operators tied together by gluons from the $\mathcal{O}\left(a^{0}\right)$ Lagrangian. ${ }^{15}$ Thus these gluons do not violate taste symmetry. The correlation between taste indices in the two four-fermion operators can only come indirectly, through the Lorentz structure.

We can check the conclusion that operators such as A35 do not appear at $\mathcal{O}\left(a^{4}\right)$ by examining the counterterms generated by other $S O(4)$-taste symmetry breaking operators. Consider four-pion vertices generated using the $\mathcal{O}\left(a^{2} p^{2}\right)$ operators from the top portion of Table XIX Form a single diagram with only four external pions by joining the four pion legs with derivatives to form a loop. The loop integral couples the indices on the derivatives and gives rise to a quartically divergent counterterm with the following four tastes of external pions: $\mu \nu, \nu \mu, \mu \sigma$, and $\sigma \mu$. This is of the same form as resulting from the $\mathcal{O}\left(a^{4}\right)$ operator that we derived above.

The general conclusion we draw from this discussion is that one cannot determine the staggered chiral Lagrangian simply by testing the invariance of all possible operators under all of the lattice symmetries. While this procedure does filter out incorrect operators, it does not lead to the correct power-counting. The only foolproof method is therefore to map each quark-level operator directly onto chiral operators.

\section{Additional source terms for vector and axial currents}

Up to this stage we have not explicitly included source terms for left- and right-handed (or equivalently vector and axial) currents. The standard method for doing so is to promote derivatives to covariant derivatives,

$$
\partial_{\mu} \Sigma \longrightarrow D_{\mu} \Sigma=\partial_{\mu} \Sigma-i \ell_{\mu} \Sigma+i \Sigma r_{\mu}, \quad \partial_{\mu} \Sigma^{\dagger} \longrightarrow D_{\mu} \Sigma^{\dagger}=\partial_{\mu} \Sigma^{\dagger}-i r_{\mu} \Sigma^{\dagger}+i \Sigma^{\dagger} \ell_{\mu},
$$

and enforce local chiral symmetry [42, 43]. Here $\ell_{\mu}$ and $r_{\mu}$ are, respectively, matrix sources for the left and righthanded currents. One also needs the field strengths associated with these sources, from which one can build contact terms at NLO. These do not contribute to S-matrix elements or decay constants, however, so we do not list them here.

15 These lead to a local operator in the chiral Lagrangian because the gluons are confined to a small region by confinement. In effect, this replaces four powers of $a$ in the eight fermion operator with $R^{4}$, where $R \sim 1 / \Lambda_{\mathrm{QCD}}$ is the confinement length scale. 


\begin{tabular}{lc}
\hline \hline Operator & Keep? \\
\hline 115. $\operatorname{Str}\left(D_{\mu} F_{L} F_{L} \Sigma D_{\mu} \Sigma^{\dagger}\right)+$ p.c. & Yes \\
116. $\operatorname{Str}\left(D_{\mu} F_{L} \Sigma D_{\mu} \Sigma^{\dagger} F_{L}\right)+$ p.c. & Sometimes \\
117. $\operatorname{Str}\left(D_{\mu} F_{L} \Sigma F_{R} D_{\mu} \Sigma^{\dagger}\right)+$ p.c. & Yes \\
118. $\operatorname{Str}\left(D_{\mu} F_{L} \Sigma D_{\mu} \Sigma^{\dagger} \Sigma F_{R} \Sigma^{\dagger}\right)+$ p.c. & Sometimes \\
\hline \hline
\end{tabular}

TABLE XV: The four $a^{2}$ source operators with one $D_{\mu} F$ and one Lie derivative. Note that the covariant derivative only acts on the object directly following. The operators which are to be kept "sometimes" can be removed using the equations of motion if the underlying operator is of type $F F(A)$.

In continuum $\chi \mathrm{PT}$, the NLO Lagrangian takes the same form as that without the sources except for the change of regular to covariant derivatives, aside from aforementioned contact terms. Possible terms involving covariant derivatives acting on $M$ or $M^{\dagger}$, for example, can be removed using the equations of motion (EoM). This turns out not to be true for $\mathrm{S} \chi \mathrm{PT}$. There are additional terms involving covariant derivatives acting on the taste spurions which cannot be removed, and which play an important role in the breaking of $S O(4)$ for axial decay constants. Of course, in addition to these terms one must change regular to covariant derivatives in all the $\mathcal{O}\left(a^{2} p^{2}\right)$ terms given previously.

The extra "source terms" are built from a covariant derivative acting on a taste spurion, a second taste spurion (since they always come in pairs), and a Lie derivative (to match the Lorentz indices). Thus they are of $\mathcal{O}\left(a^{2} p^{2}\right)$ and are produced by single insertions of four-fermion operators. We run through the possibilities in turn.

\section{a. Single insertion of $F F(A)$ operators}

We will go through the group theory and operator enumeration rather quickly, as the process is the same as in all previous sections. We will concentrate instead on eliminating operators through use of the EoM, as this is where there is a difference between staggered and continuum $\chi \mathrm{PT}$.

Operators arising from a single insertion of $[V, A \times F]$ can be built out of the left-handed basis in (A7) along with two additional elements:

$$
D_{\mu} F_{L}, \quad \Sigma D_{\mu} F_{R} \Sigma^{\dagger}
$$

Because $F_{L}$ and $F_{R}$ transform differently than $\Sigma$ and $\Sigma^{\dagger}$, the covariant derivative acts in the following way on the taste spurions:

$$
D_{\mu} F_{L}=\partial_{\mu} F_{L}-i\left[\ell_{\mu}, F_{L}\right], \quad D_{\mu} F_{R}=\partial_{\mu} F_{L}-i\left[r_{\mu}, F_{R}\right]
$$

Once $F_{L, R}$ are set to fixed taste matrices, the partial derivative pieces will vanish. However, the commutator terms can still contribute to the left- and right-handed currents, and therefore to PGB decay constants.

It is clear from th39 that taste $S$ cannot contribute, since then the commutator vanishes. Thus we need only consider tastes $P$ and $T$, in which case $F_{L}$ and $F_{R}$ are supertraceless, and transform as adjoints under the chiral symmetry group. Moreover, $D_{\mu} F_{L}$ and $D_{\mu} F_{R}$ are also supertraceless because of the cyclicity of the supertrace, so they too are adjoints. Thus operators with one taste spurion, one covariant derivative acting on a taste spurion, and one Lie derivative will correspond to the singlet irreps in the product of three adjoints. There are two singlets in this product, and therefore four operators. These operators are given in Table XV

We can remove some of these operators using the EoM. First recall (qualitatively) how this is done in the case of covariant derivatives acting on mass spurions. One such operator is $\operatorname{Str}\left(D_{\mu} M D_{\mu} \Sigma^{\dagger}\right)$. Integrating by parts, the covariant derivative can be moved onto the field, resulting in $-\operatorname{Str}\left(M D_{\mu} D_{\mu} \Sigma^{\dagger}\right)$. Then $D_{\mu} D_{\mu} \Sigma^{\dagger}$ can be replaced with operators involving either one or no derivatives acting on each of $\Sigma$ or $\Sigma^{\dagger}$ using the EoM, all of which are already included in the standard NLO effective Lagrangian. By trying to follow the same procedure for operators involving taste spurions, we will see where it breaks down.

For example, first consider operator 115. We must be careful when integrating by parts because there are more than just two terms in this operator - we cannot simply move $D_{\mu}$ from one piece to the other and add a minus sign. Derivatives can be moved around using integration by parts because we are integrating over all spacetime in the action, so total derivative terms become surface terms and can be neglected. We therefore consider a total derivative term which gives rise to operator 115:

$$
D_{\mu}\left(F_{L} F_{L} \Sigma D_{\mu} \Sigma^{\dagger}\right)=\left(D_{\mu} F_{L}\right) F_{L} \Sigma D_{\mu} \Sigma^{\dagger}+F_{L}\left(D_{\mu} F_{L}\right) \Sigma D_{\mu} \Sigma^{\dagger}+F_{L} F_{L} D_{\mu} \Sigma D_{\mu} \Sigma^{\dagger}+F_{L} F_{L} \Sigma\left(D_{\mu} D_{\mu} \Sigma^{\dagger}\right) .
$$




\begin{tabular}{lc}
\hline \hline \multicolumn{1}{c}{ Operator } & Keep? \\
\hline 119. $\operatorname{Str}\left(D_{\mu} \widetilde{F_{L}} \Sigma^{\dagger} \widetilde{\left.F_{L} D_{\mu} \Sigma^{\dagger}\right)+ \text { p.c. }}\right.$ & Yes \\
120. $\operatorname{Str}\left(D_{\mu} \widetilde{F_{L}} D_{\mu} \Sigma^{\dagger} \widetilde{F_{L}} \Sigma^{\dagger}\right)+$ p.c. & Sometimes \\
121. $\operatorname{Str}\left(D_{\mu} \widetilde{F_{L}} \Sigma^{\dagger}\right) \operatorname{Str}\left(\widetilde{F_{L}} D_{\mu} \Sigma^{\dagger}\right)+$ p.c. & Yes \\
122. $\operatorname{Str}\left(D_{\mu} \widetilde{F_{L}} D_{\mu} \Sigma^{\dagger}\right) \operatorname{Str}\left(\widetilde{F_{L}} \Sigma^{\dagger}\right)+$ p.c. & Sometimes \\
123. $\operatorname{Str}\left(D_{\mu} \widetilde{F_{L}} \widetilde{F_{R}} \Sigma D_{\mu} \Sigma^{\dagger}\right)+$ p.c. & Yes \\
124. $\operatorname{Str}\left(D_{\mu} \widetilde{F_{L}} D_{\mu} \Sigma^{\dagger} \Sigma \widetilde{F}_{R}\right)+$ p.c. & No \\
125. $\operatorname{Str}\left(D_{\mu} \widetilde{F_{L}} \Sigma^{\dagger}\right) \operatorname{Str}\left(\Sigma \widetilde{F_{R}} \Sigma D_{\mu} \Sigma^{\dagger}\right)+$ p.c. & Yes \\
126. $\operatorname{Str}\left(D_{\mu} \widetilde{F_{L}} D_{\mu} \Sigma^{\dagger}\right) \operatorname{Str}\left(\Sigma \widetilde{F_{R}}\right)+$ p.c. & Sometimes \\
\hline \hline
\end{tabular}

TABLE XVI: The eight $a^{2}$ source operators with one $D_{\mu} \widetilde{F}$ and one Lie derivative. Notation as in Table XV

After integrating over all spacetime and taking the supertrace, the l.h.s. vanishes, so the four operators on the r.h.s. are all related. The first is operator 115, while the second is operator 116. The third operator is from the LO Lagrangian, while the fourth can be related to other standard operators using the EoM. We learn two lessons from this exercise: first, that operators 115 and 116 are redundant - they are related by integration by parts; second, that we cannot remove both using the EoM - and we choose to keep operator 115. The difference from the example given above of the covariant derivative acting on the mass matrix is the presence of two taste spurions in each operator. Integration by parts can never remove the covariant derivative from both taste matrices and produce something such as $D_{\mu} D_{\mu} \Sigma$, which can be removed using EoM. The same holds for operators 117 and 118; we can remove only one of these two using the EoM, and we choose to keep operator 117.

Operators arising from a single insertion of $[S, P \times F]$ can be built similarly out of the left-handed basis in (A12) plus the elements

$$
D_{\mu} \widetilde{F_{L}} \Sigma^{\dagger}, \quad \Sigma D_{\mu} \widetilde{F_{R}}
$$

In this case $\widetilde{F_{L}}$ and $\widetilde{F_{R}}$ transform in the same way as $\Sigma$ and $\Sigma^{\dagger}$, so

$$
D_{\mu} \widetilde{F_{L}}=\partial_{\mu} \widetilde{F_{L}}-i \ell_{\mu} \widetilde{F_{L}}+i \widetilde{F_{L}} r_{\mu}, \quad D_{\mu} \widetilde{F_{R}}=\partial_{\mu} \widetilde{F_{R}}-i r_{\mu} \widetilde{F_{R}}+i \widetilde{F_{R}} \ell_{\mu}
$$

Neither $\widetilde{F_{L}} \Sigma^{\dagger}$ nor $D_{\mu} \widetilde{F_{L}} \Sigma^{\dagger}$ (or their parity conjugates) are supertraceless, so they transform as bifundamentals. Thus all operators with one $\widetilde{F}$, one $D_{\mu} \widetilde{F}$, and a Lie derivative come from singlet irreps in the product of two bifundamentals with one adjoint. There are four such singlets, and eight such operators, given in Table XVI

We can now eliminate operators using integration by parts and EoM. Operator pairs $119 \& 120,121 \& 122,123 \&$ 124 , and $124 \& 125$ are each related in this way, and we choose to keep only the first operator in each pair. In fact, operator 124 becomes equal to operator 123 once one uses the result that $D_{\mu}\left(\widetilde{F_{L}} \widetilde{F_{R}}\right)=0$, i.e. that the spurions are momentum-independent and their product has no taste. Thus we are left with four additional operators.

As in the previous sections, four fermion operators with spin $T$ do not lead to any additional mesonic operators, and simply modify some of the unknown coefficients of the spin $S$ and $P$ operators.

Inserting the appropriate taste matrices is now straightforward. We simplify expressions using the fact that $\partial_{\mu} F=0$. We also write operators in terms of $\ell_{\mu}$ and $r_{\mu}$ because the covariant derivative acts differently on the two types of taste spurions. The final "source term" operators are listed in Table XXIV

\section{b. Single insertion of $F F(B)$ operators}

In Section A3 we discussed in detail how to map onto $\mathcal{O}\left(a^{2} p^{2}\right)$ mesonic operators arising from a single insertion of a four fermion operator in $S_{6}^{F F(B)}$, so we just summarize the result here: the chiral structures are the same as those of single $F F(A)$ insertions, but the index structures are different, breaking $S O(4)$-taste and Lorentz symmetry. Simply put, each $F F(B)$ operator is identical to the corresponding $F F(A)$ operator, but with the derivative indices contracted with a pair of taste indices. However, there is one important difference: because the indices on the covariant derivative and the taste matrices are correlated, the number of operators can no longer be reduced using the EoM. Thus there are more $a^{2}$ source operators corresponding to a single insertion of $\left[V_{\mu} \times T_{\mu}\right]$ or $\left[A_{\mu} \times T_{\mu}\right]$ than those those 
from a single insertion of $[V, A \times T] .{ }^{16}$ There are, however, the same number of $a^{2}$ source operators from $\left[T_{\mu} \times V_{\mu}, A_{\mu}\right]$ as from $[S \times V, A]$ because the doubling from the inability to use the EoM is counteracted by a halving because there are only $L-R$ cross-terms. Table XXV lists all of the resulting $F F(B)$ "source term" operators.

Note that $\left[V_{\mu} \times T_{\mu}\right]$ only generates single supertrace operators, so there are no "source term" hairpins with tensor taste. This has interesting consequences for $S O(4)$-taste symmetry breaking in PGB decay constants.

\section{APPENDIX B: $\mathcal{O}\left(a^{2} p^{2}\right), \mathcal{O}\left(a^{2} m\right)$ AND $\mathcal{O}\left(a^{4}\right)$ OPERATORS IN THE S $\chi \mathcal{L}$}

[1] L. Susskind, Phys. Rev. D 16 (1977) 3031.

[2] C. Bernard et al., Nucl. Phys. Proc. Suppl. 106, 199 (2002).

[3] C. T. H. Davies et al. [HPQCD Collaboration], Phys. Rev. Lett. 92, 022001 (2004) arXiv:hep-lat/0304004.

[4] C. Aubin et al., arXiv:hep-lat/0402030

[5] B. Bunk, M. Della Morte, K. Jansen and F. Knechtli, arXiv:hep-lat/0403022

[6] A. Kennedy, plenary talk at Lattice 2004, Fermilab, USA, June 2004, to appear in the proceedings.

[7] C. Aubin et al. [MILC Collaboration], arXiv:hep-lat/0407028

[8] E. Follana, A. Hart and C. T. H. Davies [HPQCD Collaboration], arXiv:hep-lat/0406010

[9] S. Durr, C. Hoelbling and U. Wenger, arXiv:hep-lat/0406027

[10] D. H. Adams, Phys. Rev. Lett. 92, 162002 (2004) arXiv:hep-lat/0312025.

[11] D. H. Adams, arXiv:hep-lat/0409013

[12] W. Lee and S. Sharpe, Phys. Rev. D 60, 114503 (1999) arXiv:hep-lat/9905023.

[13] C. Aubin and C. Bernard, Phys. Rev. D 68, 034014 (2003) arXiv:hep-lat/0304014.

[14] C. Aubin and C. Bernard, Nucl. Phys. Proc. Suppl. 129, 182 (2004) arXiv:hep-lat/0308036.

[15] N. Ishizuka et al., Nucl. Phys. B411 (1994) 875.

[16] S. Aoki et al., Phys. Rev. D 62, 094501 (2000) arXiv:hep-lat/9912007.

\begin{tabular}{|c|c|c|c|}
\hline \multirow{2}{*}{$\begin{array}{l}\text { Generic } \\
\text { Operator }\end{array}$} & \multicolumn{3}{|c|}{ Specific $\mathcal{O}\left(a^{2} p^{2}\right)$ Operator } \\
\hline & & {$[V, A \times P]$} & {$[V, A \times T]$} \\
\hline $1+3$ & \multirow{2}{*}{\multicolumn{2}{|c|}{$\begin{array}{c}\operatorname{Str}\left(\partial_{\mu} \Sigma^{\dagger} \partial_{\mu} \Sigma \Sigma^{\dagger} \xi_{5} \Sigma \xi_{5}\right)+p . c . \\
\operatorname{Str}\left(\partial_{\mu} \Sigma^{\dagger} \xi_{5} \partial_{\mu} \Sigma \xi_{5}\right)\end{array}$}} & $\operatorname{Str}\left(\partial_{\mu} \Sigma^{\dagger} \partial_{\mu} \Sigma \Sigma^{\dagger} \xi_{\nu \rho} \Sigma \xi_{\rho \nu}\right)+$ p.c. \\
\hline 2 & & & $\operatorname{Str}\left(\partial_{\mu} \Sigma^{\dagger} \xi_{\nu \rho} \partial_{\mu} \Sigma \xi_{\rho \nu}\right)$ \\
\hline 6 & \multicolumn{2}{|r|}{$\operatorname{Str}\left(\partial_{\mu} \Sigma^{\dagger} \partial_{\mu} \Sigma\right) \operatorname{Str}\left(\xi_{5} \Sigma \xi_{5} \Sigma^{\dagger}\right)$} & $\operatorname{Str}\left(\partial_{\mu} \Sigma^{\dagger} \partial_{\mu} \Sigma\right) \operatorname{Str}\left(\xi_{\nu \rho} \Sigma \xi_{\rho \nu} \Sigma^{\dagger}\right)$ \\
\hline 7 & \multicolumn{2}{|r|}{$\operatorname{Str}\left(\Sigma \partial_{\mu} \Sigma^{\dagger} \xi_{5}\right) \operatorname{Str}\left(\Sigma^{\dagger} \partial_{\mu} \Sigma \xi_{5}\right)$} & $\operatorname{Str}\left(\Sigma \partial_{\mu} \Sigma^{\dagger} \xi_{\nu \rho}\right) \operatorname{Str}\left(\Sigma^{\dagger} \partial_{\mu} \Sigma \xi_{\rho \nu}\right)$ \\
\hline 10 & \multicolumn{2}{|r|}{$\operatorname{Str}\left(\Sigma \partial_{\mu} \Sigma^{\dagger} \xi_{5} \Sigma \partial_{\mu} \Sigma^{\dagger} \xi_{5}\right)+$ p.c. } & $\operatorname{Str}\left(\Sigma \partial_{\mu} \Sigma^{\dagger} \xi_{\nu \rho} \Sigma \partial_{\mu} \Sigma^{\dagger} \xi_{\rho \nu}\right)+$ p.c. \\
\hline 13 & \multicolumn{3}{|c|}{$\operatorname{Str}\left(\Sigma \partial_{\mu} \Sigma^{\dagger} \xi_{5}\right) \operatorname{Str}\left(\Sigma \partial_{\mu} \Sigma^{\dagger} \xi_{5}\right)+$ p.c. $\quad \operatorname{Str}\left(\Sigma \partial_{\mu} \Sigma^{\dagger} \xi_{\nu \rho}\right) \operatorname{Str}\left(\Sigma \partial_{\mu} \Sigma^{\dagger} \xi_{\rho \nu}\right)+p$} \\
\hline \multicolumn{2}{|c|}{ Generic } & \multicolumn{2}{|c|}{ Specific $\mathcal{O}\left(a^{2} m\right)$ Operator } \\
\hline \multicolumn{2}{|c|}{ Operator } & {$[V, A \times P]$} & {$[V, A \times T]$} \\
\hline \multicolumn{2}{|c|}{$15+22$} & $\operatorname{Str}\left(\xi_{5} \Sigma \xi_{5} M^{\dagger}\right)+p . c$. & $\operatorname{Str}\left(\xi_{\mu \nu} \Sigma \xi_{\nu \mu} M^{\dagger}\right)+p . c$. \\
\hline \multicolumn{2}{|c|}{$16+21$} & $\operatorname{Str}\left(\xi_{5} \Sigma M^{\dagger} \Sigma \xi_{5} \Sigma^{\dagger}\right)+$ p.c. & $\operatorname{Str}\left(\xi_{\mu \nu} \Sigma M^{\dagger} \Sigma \xi_{\nu \mu} \Sigma^{\dagger}\right)+$ p.c. \\
\hline \multicolumn{2}{|c|}{$17+23$} & $\operatorname{Str}\left(\xi_{5} \Sigma \xi_{5} \Sigma^{\dagger}\right) \operatorname{Str}\left(\Sigma M^{\dagger}\right)+$ p.c. & $\operatorname{Str}\left(\xi_{\mu \nu} \Sigma \xi_{\nu \mu} \Sigma^{\dagger}\right) \operatorname{Str}\left(\Sigma M^{\dagger}\right)+$ p.c. \\
\hline
\end{tabular}

TABLE XVII: Operators in the staggered chiral Lagrangian arising from a single insertion of an $S_{6}^{F F(A)}$ operator with spin $V$ or $A$. Implicit sums over repeated indices follow the summation convention defined in (1), and p.c. indicates parity conjugate. Each chiral operator has an independent undetermined coefficient of $\mathcal{O}\left(a^{2}\right)$. Partial derivatives act by convention only on the object immediately to their right. To include left- and right-handed sources, derivatives should be replaced with covariant derivatives. $M$ and $M^{\dagger}$ serve as (pseudo)scalar sources; if such sources are absent then $M=M^{\dagger}=\mathcal{M}$.

16 Note that the operators resulting from generic forms 115 and 116 are the same up to a sign, so the doubling of operators does not apply in this case. 


\begin{tabular}{|c|c|c|}
\hline \multirow{2}{*}{$\begin{array}{l}\text { Generic } \\
\text { Operator }\end{array}$} & \multicolumn{2}{|c|}{ Specific $\mathcal{O}\left(a^{2} p^{2}\right)$ Operator } \\
\hline & {$[S, P \times V]$} & {$[S, P \times A]$} \\
\hline 36 & $\operatorname{Str}\left(\Sigma \partial_{\mu} \Sigma^{\dagger} \xi_{\nu} \Sigma^{\dagger} \partial_{\mu} \Sigma \xi_{\nu}\right)$ & $\operatorname{Str}\left(\Sigma \partial_{\mu} \Sigma^{\dagger} \xi_{\nu 5} \Sigma^{\dagger} \partial_{\mu} \Sigma \xi_{5 \nu}\right)$ \\
\hline $38+39$ & $\operatorname{Str}\left(\partial_{\mu} \Sigma^{\dagger} \partial_{\mu} \Sigma \Sigma^{\dagger} \xi_{\nu}\right) \operatorname{Str}\left(\Sigma \xi_{\nu}\right)+$ p.c. & $\operatorname{Str}\left(\partial_{\mu} \Sigma^{\dagger} \partial_{\mu} \Sigma \Sigma^{\dagger} \xi_{\nu 5}\right) \operatorname{Str}\left(\Sigma \xi_{5 \nu}\right)+$ p.c. \\
\hline 41 & $\operatorname{Str}\left(\partial_{\mu} \Sigma^{\dagger} \xi_{\nu}\right) \operatorname{Str}\left(\partial_{\mu} \Sigma \xi_{\nu}\right)$ & $\operatorname{Str}\left(\partial_{\mu} \Sigma^{\dagger} \xi_{\nu 5}\right) \operatorname{Str}\left(\partial_{\mu} \Sigma \xi_{5 \nu}\right)$ \\
\hline 42 & $\operatorname{Str}\left(\partial_{\mu} \Sigma^{\dagger} \partial_{\mu} \Sigma\right) \operatorname{Str}\left(\xi_{\nu} \Sigma^{\dagger}\right) \operatorname{Str}\left(\Sigma \xi_{\nu}\right)$ & $\operatorname{Str}\left(\partial_{\mu} \Sigma^{\dagger} \partial_{\mu} \Sigma\right) \operatorname{Str}\left(\xi_{\nu 5} \Sigma^{\dagger}\right) \operatorname{Str}\left(\Sigma \xi_{5 \nu}\right)$ \\
\hline 43 & $\operatorname{Str}\left(\partial_{\mu} \Sigma \partial_{\mu} \Sigma^{\dagger} \xi_{\nu} \Sigma^{\dagger} \xi_{\nu} \Sigma^{\dagger}\right)+$ p.c. & $\operatorname{Str}\left(\partial_{\mu} \Sigma \partial_{\mu} \Sigma^{\dagger} \xi_{\nu 5} \Sigma^{\dagger} \xi_{5 \nu} \Sigma^{\dagger}\right)+$ p.c. \\
\hline 44 & $\operatorname{Str}\left(\partial_{\mu} \Sigma^{\dagger} \xi_{\nu} \partial_{\mu} \Sigma^{\dagger} \xi_{\nu}\right)+$ p.c. & $\operatorname{Str}\left(\partial_{\mu} \Sigma^{\dagger} \xi_{\nu 5} \partial_{\mu} \Sigma^{\dagger} \xi_{5 \nu}\right)+$ p.c. \\
\hline 45 & $\operatorname{Str}\left(\partial_{\mu} \Sigma^{\dagger} \partial_{\mu} \Sigma\right) \operatorname{Str}\left(\xi_{\nu} \Sigma^{\dagger} \xi_{\nu} \Sigma^{\dagger}\right)+$ p.c. & $\operatorname{Str}\left(\partial_{\mu} \Sigma^{\dagger} \partial_{\mu} \Sigma\right) \operatorname{Str}\left(\xi_{\nu 5} \Sigma^{\dagger} \xi_{5 \nu} \Sigma^{\dagger}\right)+p . c$. \\
\hline 46 & $\operatorname{Str}\left(\partial_{\mu} \Sigma \partial_{\mu} \Sigma^{\dagger} \xi_{\nu} \Sigma^{\dagger}\right) \operatorname{Str}\left(\xi_{\nu} \Sigma^{\dagger}\right)+$ p.c. & $\operatorname{Str}\left(\partial_{\mu} \Sigma \partial_{\mu} \Sigma^{\dagger} \xi_{\nu 5} \Sigma^{\dagger}\right) \operatorname{Str}\left(\xi_{5 \nu} \Sigma^{\dagger}\right)+p . c$. \\
\hline 47 & $\operatorname{Str}\left(\partial_{\mu} \Sigma^{\dagger} \xi_{\nu}\right)^{2}+$ p.c. & $\operatorname{Str}\left(\partial_{\mu} \Sigma^{\dagger} \xi_{\nu 5}\right) \operatorname{Str}\left(\partial_{\mu} \Sigma^{\dagger} \xi_{5 \nu}\right)+$ p.c. \\
\hline 48 & $\operatorname{Str}\left(\partial_{\mu} \Sigma^{\dagger} \partial_{\mu} \Sigma\right) \operatorname{Str}\left(\xi_{\nu} \Sigma^{\dagger}\right)^{2}+$ p.c. & $\operatorname{Str}\left(\partial_{\mu} \Sigma^{\dagger} \partial_{\mu} \Sigma\right) \operatorname{Str}\left(\xi_{\nu 5} \Sigma^{\dagger}\right) \operatorname{Str}\left(\xi_{5 \nu} \Sigma^{\dagger}\right)+$ p.c. \\
\hline Generic & \multicolumn{2}{|c|}{ Specific $\mathcal{O}\left(a^{2} m\right)$ Operator } \\
\hline Operator & {$[S, P \times V]$} & {$[S, P \times A]$} \\
\hline $52+59$ & $\operatorname{Str}\left(\xi_{\mu} M^{\dagger}\right) \operatorname{Str}\left(\Sigma \xi_{\mu}\right)$ & $\operatorname{Str}\left(\xi_{\mu 5} M^{\dagger}\right) \operatorname{Str}\left(\Sigma \xi_{5 \mu}\right)$ \\
\hline $53+58$ & $\operatorname{Str}\left(\xi_{\mu} \Sigma^{\dagger}\right) \operatorname{Str}\left(\Sigma \xi_{\mu} \Sigma M^{\dagger}\right)+$ p.c. & $\operatorname{Str}\left(\xi_{\mu 5} \Sigma^{\dagger}\right) \operatorname{Str}\left(\Sigma \xi_{5 \mu} \Sigma M^{\dagger}\right)+$ p.c. \\
\hline $54+60$ & $\operatorname{Str}\left(\xi_{\mu} \Sigma^{\dagger}\right) \operatorname{Str}\left(\Sigma \xi_{\mu}\right) \operatorname{Str}\left(\Sigma M^{\dagger}\right)+$ p.c. & $\operatorname{Str}\left(\xi_{\mu 5} \Sigma^{\dagger}\right) \operatorname{Str}\left(\Sigma \xi_{5 \mu}\right) \operatorname{Str}\left(\Sigma M^{\dagger}\right)+$ p.c. \\
\hline 61 & $\operatorname{Str}\left(\xi_{\mu} \Sigma^{\dagger} \xi_{\mu} M^{\dagger}\right)+$ p.c. & $\operatorname{Str}\left(\xi_{\mu 5} \Sigma^{\dagger} \xi_{5 \mu} M^{\dagger}\right)+p . c$. \\
\hline 62 & $\operatorname{Str}\left(\xi_{\mu} \Sigma^{\dagger} \xi_{\mu} \Sigma^{\dagger}\right) \operatorname{Str}\left(\Sigma M^{\dagger}\right)+$ p.c. & $\operatorname{Str}\left(\xi_{\mu 5} \Sigma^{\dagger} \xi_{5 \mu} \Sigma^{\dagger}\right) \operatorname{Str}\left(\Sigma M^{\dagger}\right)+$ p.c. \\
\hline 63 & $\operatorname{Str}\left(\xi_{\mu} \Sigma^{\dagger}\right) \operatorname{Str}\left(\xi_{\mu} M^{\dagger}\right)+$ p.c. & $\operatorname{Str}\left(\xi_{\mu 5} \Sigma^{\dagger}\right) \operatorname{Str}\left(\xi_{5 \mu} M^{\dagger}\right)+$ p.c. \\
\hline 64 & $\operatorname{Str}\left(\xi_{\mu} \Sigma^{\dagger}\right) \operatorname{Str}\left(\xi_{\mu} \Sigma^{\dagger}\right) \operatorname{Str}\left(\Sigma M^{\dagger}\right)+$ p.c. & $\operatorname{Str}\left(\xi_{\mu 5} \Sigma^{\dagger}\right) \operatorname{Str}\left(\xi_{5 \mu} \Sigma^{\dagger}\right) \operatorname{Str}\left(\Sigma M^{\dagger}\right)+$ p.c. \\
\hline 65 & $\operatorname{Str}\left(\xi_{\mu} \Sigma^{\dagger} \xi_{\mu} \Sigma^{\dagger} M \Sigma^{\dagger}\right)+p . c$. & $\operatorname{Str}\left(\xi_{\mu 5} \Sigma^{\dagger} \xi_{5 \mu} \Sigma^{\dagger} M \Sigma^{\dagger}\right)+$ p.c. \\
\hline 66 & $\operatorname{Str}\left(\xi_{\mu} \Sigma^{\dagger} \xi_{\mu} \Sigma^{\dagger}\right) \operatorname{Str}\left(M \Sigma^{\dagger}\right)+$ p.c. & $\operatorname{Str}\left(\xi_{\mu 5} \Sigma^{\dagger} \xi_{5 \mu} \Sigma^{\dagger}\right) \operatorname{Str}\left(M \Sigma^{\dagger}\right)+$ p.c. \\
\hline 67 & $\operatorname{Str}\left(\xi_{\mu} \Sigma^{\dagger}\right) \operatorname{Str}\left(\xi_{\mu} \Sigma^{\dagger} M \Sigma^{\dagger}\right)+$ p.c. & $\operatorname{Str}\left(\xi_{\mu 5} \Sigma^{\dagger}\right) \operatorname{Str}\left(\xi_{5 \mu} \Sigma^{\dagger} M \Sigma^{\dagger}\right)+$ p.c. \\
\hline 68 & $\operatorname{Str}\left(\xi_{\mu} \Sigma^{\dagger}\right) \operatorname{Str}\left(\xi_{\mu} \Sigma^{\dagger}\right) \operatorname{Str}\left(M \Sigma^{\dagger}\right)+$ p.c. & $\operatorname{Str}\left(\xi_{\mu 5} \Sigma^{\dagger}\right) \operatorname{Str}\left(\xi_{5 \mu} \Sigma^{\dagger}\right) \operatorname{Str}\left(M \Sigma^{\dagger}\right)+$ p.c. \\
\hline
\end{tabular}

TABLE XVIII: Operators in the staggered chiral Lagrangian arising from a single insertion of an $S_{6}^{F F(A)}$ operator with spin $S$ or $P$. Notation as in table XVII

[17] K. Orginos et al., Phys. Rev. D 59, 014501 (1999) arXiv:hep-lat/9805009.

[18] C. Bernard et al., Nucl. Phys. Proc. Suppl. 119, 170 (2003) arXiv:hep-lat/0209086.

[19] C. Aubin and C. Bernard, Phys. Rev. D 68, 074011 (2003) arXiv:hep-lat/0306026 .

[20] S. R. Sharpe and R. J. Singleton, Phys. Rev. D 58, 074501 (1998) arXiv:hep-lat/9804028.

[21] K. Symanzik, Nucl. Phys. B226 (1983) 187, 205.

[22] M. F. L. Golterman and J. Smit, Nucl. Phys. B 245, 61 (1984); B 255, 328 (1985).

[23] D. Verstegen, Nucl. Phys. B249 (1985) 685.

[24] S. Sharpe, Nucl. Physics B (Proc. Suppl.) 34 (1994) 403 arXiv:hep-lat/9312009

[25] Y. Luo, Phys. Rev. D 55353 (1997).

[26] Y. Luo, Phys. Rev. D 57 265, 1998 arXiv:hep-lat/9702013.

[27] D. Daniel and S. Sheard, Nucl. Phys. B302 (1988) 471.

[28] A. Patel and S. Sharpe, Nucl. Phys. B395 (1993) 701 arXiv:hep-lat/9210039.

[29] S. Sharpe and A. Patel, Nucl. Phys. B417 (1994) 307 arXiv:hep-lat/9310004.

[30] G. Kilcup and S. Sharpe, Nucl. Phys. B283 (1987) 493.

[31] C. Bernard, Phys. Rev. D 65, 054031 (2002) arXiv:hep-lat/0111051.

[32] S. R. Sharpe and N. Shoresh, Phys. Rev. D 62, 094503 (2000) arXiv:hep-lat/0006017.

[33] C. W. Bernard and M. F. Golterman, Phys. Rev. D 49, 486 (1994) arXiv:hep-lat/9306005.

[34] S. R. Sharpe and N. Shoresh, Phys. Rev. D 64, 114510 (2001) arXiv:hep-lat/0108003.

[35] W. A. Bardeen, E. Eichten and H. Thacker, Phys. Rev. D 69, 054502 (2004) arXiv:hep-lat/0307023.

[36] S. R. Sharpe and R. S. Van de Water, Phys. Rev. D 69, 054027 (2004) arXiv:hep-lat/0310012.

[37] M. F. L. Golterman, Nucl. Phys. B273, 663 (1986).

[38] A. B. Balantekin and I. Bars, J. Math. Phys. 22, 1149 (1981).

[39] A. B. Balantekin and I. Bars, J. Math. Phys. 22, 1810 (1981). 


\begin{tabular}{l|c}
\hline \hline Generic & Specific $\mathcal{O}\left(a^{2} p^{2}\right)$ Operator \\
Operator & {$\left[V_{\mu} \times T_{\mu}\right]$ and $\left[A_{\mu} \times T_{\mu}\right]$} \\
\hline $1+3$ & $\operatorname{Str}\left(\partial_{\mu} \Sigma^{\dagger} \partial_{\mu} \Sigma \Sigma^{\dagger} \xi_{\mu \nu} \Sigma \xi_{\nu \mu}\right)+p . c$. \\
2 & $\operatorname{Str}\left(\partial_{\mu} \Sigma^{\dagger} \xi_{\mu \nu} \partial_{\mu} \Sigma \xi_{\nu \mu}\right)$ \\
6 & $\operatorname{Str}\left(\partial_{\mu} \Sigma^{\dagger} \partial_{\mu} \Sigma\right) \operatorname{Str}\left(\xi_{\mu \nu} \Sigma \xi_{\nu \mu} \Sigma^{\dagger}\right)$ \\
7 & $\operatorname{Str}\left(\Sigma \partial_{\mu} \Sigma^{\dagger} \xi_{\mu \nu}\right) \operatorname{Str}\left(\Sigma^{\dagger} \partial_{\mu} \Sigma \xi_{\nu \mu}\right)$ \\
10 & $\operatorname{Str}\left(\Sigma \partial_{\mu} \Sigma^{\dagger} \xi_{\mu \nu} \Sigma \partial_{\mu} \Sigma^{\dagger} \xi_{\nu \mu}\right)+p . c$. \\
13 & $\operatorname{Str}\left(\Sigma \partial_{\mu} \Sigma^{\dagger} \xi_{\mu \nu}\right) \operatorname{Str}\left(\Sigma \partial_{\mu} \Sigma^{\dagger} \xi_{\nu \mu}\right)+p . c$. \\
\hline \hline
\end{tabular}

\begin{tabular}{l|cc}
\hline \hline Generic & Specific $\mathcal{O}\left(a^{2} p^{2}\right)$ Operator \\
Operator & {$\left[T_{\mu} \times V_{\mu}\right]$} & {$\left[T_{\mu} \times A_{\mu}\right]$} \\
\hline 36 & $\operatorname{Str}\left(\Sigma \partial_{\mu} \Sigma^{\dagger} \xi_{\mu} \Sigma^{\dagger} \partial_{\mu} \Sigma \xi_{\mu}\right)$ & $\operatorname{Str}\left(\Sigma \partial_{\mu} \Sigma^{\dagger} \xi_{\mu 5} \Sigma^{\dagger} \partial_{\mu} \Sigma \xi_{5 \mu}\right)$ \\
$38+39$ & $\operatorname{Str}\left(\partial_{\mu} \Sigma^{\dagger} \partial_{\mu} \Sigma \Sigma^{\dagger} \xi_{\mu}\right) \operatorname{Str}\left(\Sigma \xi_{\mu}\right)+$ p.c. & $\operatorname{Str}\left(\partial_{\mu} \Sigma^{\dagger} \partial_{\mu} \Sigma \Sigma^{\dagger} \xi_{\mu 5}\right) \operatorname{Str}\left(\Sigma \xi_{5 \mu}\right)+$ p.c. \\
41 & $\operatorname{Str}\left(\partial_{\mu} \Sigma^{\dagger} \xi_{\mu}\right) \operatorname{Str}\left(\partial_{\mu} \Sigma \xi_{\mu}\right)$ & $\operatorname{Str}\left(\partial_{\mu} \Sigma^{\dagger} \xi_{\mu 5}\right) \operatorname{Str}\left(\partial_{\mu} \Sigma \xi_{5 \mu}\right)$ \\
42 & $\operatorname{Str}\left(\partial_{\mu} \Sigma^{\dagger} \partial_{\mu} \Sigma\right) \operatorname{Str}\left(\xi_{\mu} \Sigma^{\dagger}\right) \operatorname{Str}\left(\Sigma \xi_{\mu}\right)$ & $\operatorname{Str}\left(\partial_{\mu} \Sigma^{\dagger} \partial_{\mu} \Sigma\right) \operatorname{Str}\left(\xi_{\mu 5} \Sigma^{\dagger}\right) \operatorname{Str}\left(\Sigma \xi_{5 \mu}\right)$ \\
\hline \hline
\end{tabular}

TABLE XIX: Rotationally non-invariant operators in the staggered chiral Lagrangian arising from a single insertion of $S_{6}^{F F(B)}$ operators. There is an implicit summation over both $\mu$ and $\nu$ with the constraint $\nu \neq \mu$.

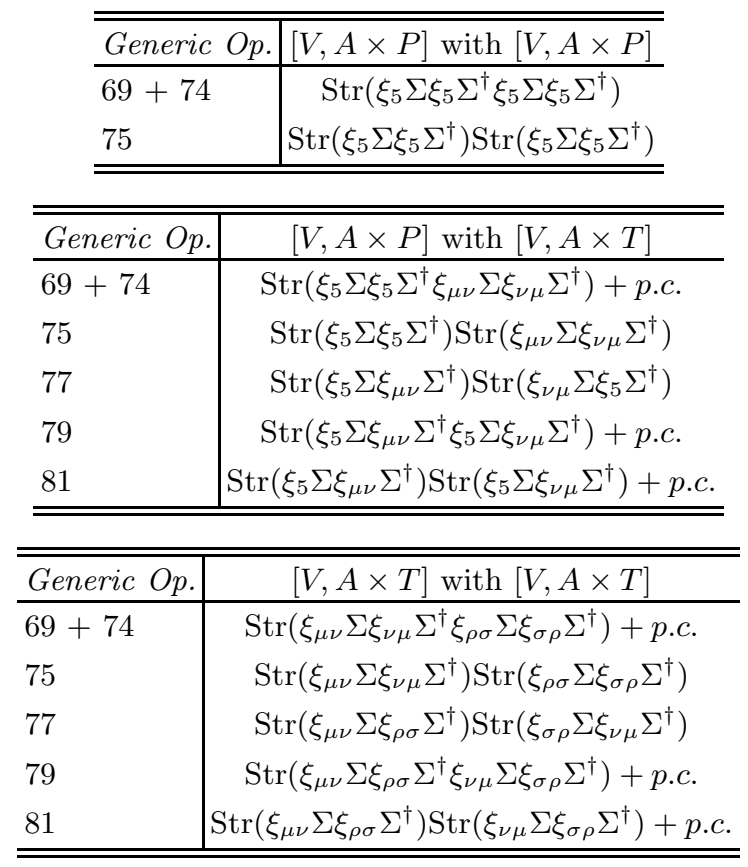

TABLE XX: $\mathcal{O}\left(a^{4}\right)$ operators in the staggered chiral Lagrangian arising from two insertions of an $S_{6}^{F F(A)}$ operator with spin $V$ or $A$. Notation as in table XVII

[40] H. W. Fearing and S. Scherer Phys. Rev. D 53, 315 (1996) arXiv:hep-ph/9408346.

[41] J. Bijnens, G. Colangelo, and G. Ecker, JHEP 9902, 020 (1999) arXiv:hep-ph/9902437.

[42] J. Gasser and H. Leutwyler, Ann. Phys. 158, 142 (1984).

[43] J. Gasser and H. Leutwyler, Nucl. Phys. B250, 465 (1985). 


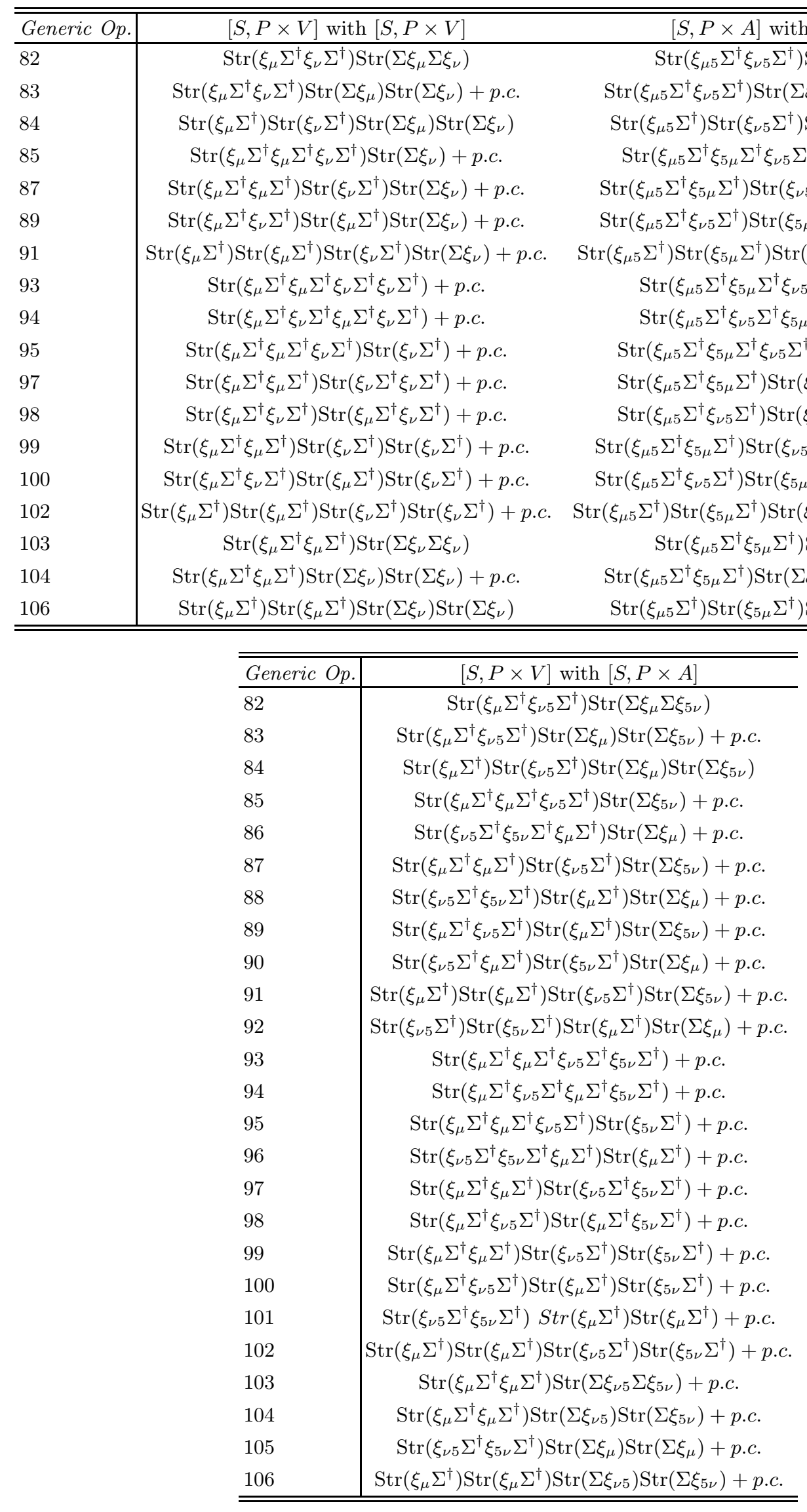

TABLE XXI: $\mathcal{O}\left(a^{4}\right)$ operators in the staggered chiral Lagrangian arising from two insertions of an $S_{6}^{F F(A)}$ operator with spin $S$ or $P$. Notation as in table XVII 


\begin{tabular}{|c|c|c|}
\hline Generic Op. & {$[V, A \times P]$ with $[S, P \times V]$} & {$[V, A \times P]$ with $[S, P \times A]$} \\
\hline 107 & $\operatorname{Str}\left(\xi_{5} \Sigma \xi_{\mu} \Sigma \xi_{5} \Sigma^{\dagger} \xi_{\mu} \Sigma^{\dagger}\right)$ & $\operatorname{Str}\left(\xi_{5} \Sigma \xi_{\mu 5} \Sigma \xi_{5} \Sigma^{\dagger} \xi_{5 \mu} \Sigma^{\dagger}\right)$ \\
\hline $108+109$ & $\operatorname{Str}\left(\xi_{5} \Sigma \xi_{\mu} \Sigma \xi_{5} \Sigma^{\dagger}\right) \operatorname{Str}\left(\xi_{\mu} \Sigma^{\dagger}\right)+$ p.c. & $\operatorname{Str}\left(\xi_{5} \Sigma \xi_{\mu 5} \Sigma \xi_{5} \Sigma^{\dagger}\right) \operatorname{Str}\left(\xi_{5 \mu} \Sigma^{\dagger}\right)+$ p.c. \\
\hline 110 & $\operatorname{Str}\left(\xi_{5} \Sigma \xi_{5} \Sigma^{\dagger}\right) \operatorname{Str}\left(\xi_{\mu} \Sigma^{\dagger}\right) \operatorname{Str}\left(\Sigma \xi_{\mu}\right)$ & $\operatorname{Str}\left(\xi_{5} \Sigma \xi_{5} \Sigma^{\dagger}\right) \operatorname{Str}\left(\xi_{\mu 5} \Sigma^{\dagger}\right) \operatorname{Str}\left(\Sigma \xi_{5 \mu}\right)$ \\
\hline 111 & $\operatorname{Str}\left(\xi_{\mu} \Sigma^{\dagger} \xi_{\mu} \Sigma^{\dagger} \xi_{5} \Sigma \xi_{5} \Sigma^{\dagger}\right)+$ p.c. & $\operatorname{Str}\left(\xi_{\mu 5} \Sigma^{\dagger} \xi_{5 \mu} \Sigma^{\dagger} \xi_{5} \Sigma \xi_{5} \Sigma^{\dagger}\right)+$ p.c. \\
\hline 112 & $\operatorname{Str}\left(\xi_{\mu} \Sigma^{\dagger} \xi_{\mu} \Sigma^{\dagger}\right) \operatorname{Str}\left(\xi_{5} \Sigma \xi_{5} \Sigma^{\dagger}\right)+$ p.c. & $\operatorname{Str}\left(\xi_{\mu 5} \Sigma^{\dagger} \xi_{5 \mu} \Sigma^{\dagger}\right) \operatorname{Str}\left(\xi_{5} \Sigma \xi_{5} \Sigma^{\dagger}\right)+$ p.c. \\
\hline 113 & $\operatorname{Str}\left(\xi_{\mu} \Sigma^{\dagger} \xi_{5} \Sigma \xi_{5} \Sigma^{\dagger}\right) \operatorname{Str}\left(\xi_{\mu} \Sigma^{\dagger}\right)+$ p.c. & $\operatorname{Str}\left(\xi_{\mu 5} \Sigma^{\dagger} \xi_{5} \Sigma \xi_{5} \Sigma^{\dagger}\right) \operatorname{Str}\left(\xi_{5 \mu} \Sigma^{\dagger}\right)+$ p.c. \\
\hline 114 & $\operatorname{Str}\left(\xi_{\mu} \Sigma^{\dagger}\right) \operatorname{Str}\left(\xi_{\mu} \Sigma^{\dagger}\right) \operatorname{Str}\left(\xi_{5} \Sigma \xi_{5} \Sigma^{\dagger}\right)+$ p.c. & $\operatorname{Str}\left(\xi_{\mu 5} \Sigma^{\dagger}\right) \operatorname{Str}\left(\xi_{5 \mu} \Sigma^{\dagger}\right) \operatorname{Str}\left(\xi_{5} \Sigma \xi_{5} \Sigma^{\dagger}\right)+$ p.c. \\
\hline Generic Op. & {$[V, A \times T]$ with $[S, P \times V]$} & 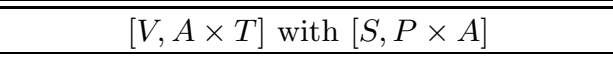 \\
\hline 107 & $\operatorname{Str}\left(\xi_{\mu \nu} \Sigma \xi_{\rho} \Sigma \xi_{\nu \mu} \Sigma^{\dagger} \xi_{\rho} \Sigma^{\dagger}\right)$ & $\operatorname{Str}\left(\xi_{\mu \nu} \Sigma \xi_{\rho 5} \Sigma \xi_{\nu \mu} \Sigma^{\dagger} \xi_{5 \rho} \Sigma^{\dagger}\right)$ \\
\hline $108+109$ & $\operatorname{Str}\left(\xi_{\mu \nu} \Sigma \xi_{\rho} \Sigma \xi_{\nu \mu} \Sigma^{\dagger}\right) \operatorname{Str}\left(\xi_{\rho} \Sigma^{\dagger}\right)+$ p.c. & $\operatorname{Str}\left(\xi_{\mu \nu} \Sigma \xi_{\rho 5} \Sigma \xi_{\nu \mu} \Sigma^{\dagger}\right) \operatorname{Str}\left(\xi_{5 \rho} \Sigma^{\dagger}\right)+$ p.c. \\
\hline 110 & $\operatorname{Str}\left(\xi_{\mu \nu} \Sigma \xi_{\nu \mu} \Sigma^{\dagger}\right) \operatorname{Str}\left(\xi_{\rho} \Sigma^{\dagger}\right) \operatorname{Str}\left(\Sigma \xi_{\rho}\right)$ & $\operatorname{Str}\left(\xi_{\mu \nu} \Sigma \xi_{\nu \mu} \Sigma^{\dagger}\right) \operatorname{Str}\left(\xi_{\rho 5} \Sigma^{\dagger}\right) \operatorname{Str}\left(\Sigma \xi_{5 \rho}\right)$ \\
\hline 111 & $\operatorname{Str}\left(\xi_{\rho} \Sigma^{\dagger} \xi_{\rho} \Sigma^{\dagger} \xi_{\mu \nu} \Sigma \xi_{\nu \mu} \Sigma^{\dagger}\right)+$ p.c. & $\operatorname{Str}\left(\xi_{\rho 5} \Sigma^{\dagger} \xi_{5 \rho} \Sigma^{\dagger} \xi_{\mu \nu} \Sigma \xi_{\nu \mu} \Sigma^{\dagger}\right)+$ p.c. \\
\hline 112 & $\operatorname{Str}\left(\xi_{\rho} \Sigma^{\dagger} \xi_{\rho} \Sigma^{\dagger}\right) \operatorname{Str}\left(\xi_{\mu \nu} \Sigma \xi_{\nu \mu} \Sigma^{\dagger}\right)+$ p.c. & $\operatorname{Str}\left(\xi_{\rho 5} \Sigma^{\dagger} \xi_{5 \rho} \Sigma^{\dagger}\right) \operatorname{Str}\left(\xi_{\mu \nu} \Sigma \xi_{\nu \mu} \Sigma^{\dagger}\right)+$ p.c. \\
\hline 113 & $\operatorname{Str}\left(\xi_{\rho} \Sigma^{\dagger} \xi_{\mu \nu} \Sigma \xi_{\nu \mu} \Sigma^{\dagger}\right) \operatorname{Str}\left(\xi_{\rho} \Sigma^{\dagger}\right)+$ p.c. & $\operatorname{Str}\left(\xi_{\rho 5} \Sigma^{\dagger} \xi_{\mu \nu} \Sigma \xi_{\nu \mu} \Sigma^{\dagger}\right) \operatorname{Str}\left(\xi_{5 \rho} \Sigma^{\dagger}\right)+$ p.c. \\
\hline 114 & $\operatorname{Str}\left(\xi_{\rho} \Sigma^{\dagger}\right) \operatorname{Str}\left(\xi_{\rho} \Sigma^{\dagger}\right) \operatorname{Str}\left(\xi_{\mu \nu} \Sigma \xi_{\nu \mu} \Sigma^{\dagger}\right)+$ p.c. & $\operatorname{Str}\left(\xi_{\rho 5} \Sigma^{\dagger}\right) \operatorname{Str}\left(\xi_{5 \rho} \Sigma^{\dagger}\right) \operatorname{Str}\left(\xi_{\mu \nu} \Sigma \xi_{\nu \mu} \Sigma^{\dagger}\right)+$ p.c. \\
\hline
\end{tabular}

TABLE XXII: $\mathcal{O}\left(a^{4}\right)$ operators in the staggered chiral Lagrangian arising from two insertions of $S_{6}^{F F(A)}$ operators, one with spin $V$ or $A$, the other with spin $S$ or $P$. Notation as in table XVII

\begin{tabular}{l|cc}
\hline \hline Generic Op. & {$\left[T_{\mu} \times V_{\mu}\right]$ with $\left[T_{\mu} \times V_{\mu}\right]$} & {$\left[T_{\mu} \times A_{\mu}\right]$ with $\left[T_{\mu} \times A_{\mu}\right]$} \\
\hline 82 & $\operatorname{Str}\left(\xi_{\mu} \Sigma^{\dagger} \xi_{\mu} \Sigma^{\dagger}\right) \operatorname{Str}\left(\Sigma \xi_{\mu} \Sigma \xi_{\mu}\right)$ & $\operatorname{Str}\left(\xi_{\mu 5} \Sigma^{\dagger} \xi_{\mu 5} \Sigma^{\dagger}\right) \operatorname{Str}\left(\Sigma \xi_{5 \mu} \Sigma \xi_{5 \mu}\right)$ \\
83 & $\operatorname{Str}\left(\xi_{\mu} \Sigma^{\dagger} \xi_{\mu} \Sigma^{\dagger}\right) \operatorname{Str}\left(\Sigma \xi_{\mu}\right) \operatorname{Str}\left(\Sigma \xi_{\mu}\right)+p . c$. & $\operatorname{Str}\left(\xi_{\mu 5} \Sigma^{\dagger} \xi_{\mu 5} \Sigma^{\dagger}\right) \operatorname{Str}\left(\Sigma \xi_{5 \mu}\right) \operatorname{Str}\left(\Sigma \xi_{5 \mu}\right)+p . c$. \\
84 & $\operatorname{Str}\left(\xi_{\mu} \Sigma^{\dagger}\right) \operatorname{Str}\left(\xi_{\mu} \Sigma^{\dagger}\right) \operatorname{Str}\left(\Sigma \xi_{\mu}\right) \operatorname{Str}\left(\Sigma \xi_{\mu}\right)$ & $\operatorname{Str}\left(\xi_{\mu 5} \Sigma^{\dagger}\right) \operatorname{Str}\left(\xi_{\mu 5} \Sigma^{\dagger}\right) \operatorname{Str}\left(\Sigma \xi_{5 \mu}\right) \operatorname{Str}\left(\Sigma \xi_{5 \mu}\right)$ \\
\hline \hline
\end{tabular}

\begin{tabular}{l|c}
\hline \hline Generic Op. & {$\left[T_{\mu} \times V_{\mu}\right]$ with $\left[T_{\mu} \times A_{\mu}\right]$} \\
\hline 82 & $\operatorname{Str}\left(\xi_{\mu} \Sigma^{\dagger} \xi_{\mu 5} \Sigma^{\dagger}\right) \operatorname{Str}\left(\Sigma \xi_{\mu} \Sigma \xi_{5 \mu}\right)$ \\
83 & $\operatorname{Str}\left(\xi_{\mu} \Sigma^{\dagger} \xi_{\mu 5} \Sigma^{\dagger}\right) \operatorname{Str}\left(\Sigma \xi_{\mu}\right) \operatorname{Str}\left(\Sigma \xi_{5 \mu}\right)+$ p.c. \\
84 & $\operatorname{Str}\left(\xi_{\mu} \Sigma^{\dagger}\right) \operatorname{Str}\left(\xi_{\mu 5} \Sigma^{\dagger}\right) \operatorname{Str}\left(\Sigma \xi_{\mu}\right) \operatorname{Str}\left(\Sigma \xi_{5 \mu}\right)$ \\
\hline \hline
\end{tabular}

\begin{tabular}{l|cc}
\hline \hline Generic Op. & {$\left[V_{\mu}, A_{\mu} \times T_{\mu}\right]$ with $\left[T_{\mu} \times V_{\mu}\right]$} & {$\left[V_{\mu}, A_{\mu} \times T_{\mu}\right]$ with $\left[T_{\mu} \times A_{\mu}\right]$} \\
\hline 107 & $\operatorname{Str}\left(\xi_{\mu \nu} \Sigma \xi_{\mu} \Sigma \xi_{\nu \mu} \Sigma^{\dagger} \xi_{\mu} \Sigma^{\dagger}\right)$ & $\operatorname{Str}\left(\xi_{\mu \nu} \Sigma \xi_{\mu 5} \Sigma \xi_{\nu \mu} \Sigma^{\dagger} \xi_{5 \mu} \Sigma^{\dagger}\right)$ \\
$108+109$ & $\operatorname{Str}\left(\xi_{\mu \nu} \Sigma \xi_{\mu} \Sigma \xi_{\nu \mu} \Sigma^{\dagger}\right) \operatorname{Str}\left(\xi_{\mu} \Sigma^{\dagger}\right)+$ p.c. & $\operatorname{Str}\left(\xi_{\mu \nu} \Sigma \xi_{\mu 5} \Sigma \xi_{\nu \mu} \Sigma^{\dagger}\right) \operatorname{Str}\left(\xi_{5 \mu} \Sigma^{\dagger}\right)+$ p.c. \\
110 & $\operatorname{Str}\left(\xi_{\mu \nu} \Sigma \xi_{\nu \mu} \Sigma^{\dagger}\right) \operatorname{Str}\left(\xi_{\mu} \Sigma^{\dagger}\right) \operatorname{Str}\left(\Sigma \xi_{\mu}\right)$ & $\operatorname{Str}\left(\xi_{\mu \nu} \Sigma \xi_{\nu \mu} \Sigma^{\dagger}\right) \operatorname{Str}\left(\xi_{\mu 5} \Sigma^{\dagger}\right) \operatorname{Str}\left(\Sigma \xi_{5 \mu}\right)$ \\
\hline \hline
\end{tabular}

\begin{tabular}{l|c}
\hline \hline Generic Op. & {$\left[V_{\mu}, A_{\mu} \times T_{\mu}\right]$ with $\left[V_{\mu}, A_{\mu} \times T_{\mu}\right]$} \\
\hline $69+74$ & $\operatorname{Str}\left(\xi_{\mu \nu} \Sigma \xi_{\nu \mu} \Sigma^{\dagger} \xi_{\mu \sigma} \Sigma \xi_{\sigma \mu} \Sigma^{\dagger}\right)+p . c$. \\
75 & $\operatorname{Str}\left(\xi_{\mu \nu} \Sigma \xi_{\nu \mu} \Sigma^{\dagger}\right) \operatorname{Str}\left(\xi_{\mu \sigma} \Sigma \xi_{\sigma \mu} \Sigma^{\dagger}\right)$ \\
77 & $\operatorname{Str}\left(\xi_{\mu \nu} \Sigma \xi_{\mu \sigma} \Sigma^{\dagger}\right) \operatorname{Str}\left(\xi_{\sigma \mu} \Sigma \xi_{\nu \mu} \Sigma^{\dagger}\right)$ \\
79 & $\operatorname{Str}\left(\xi_{\mu \nu} \Sigma \xi_{\mu \sigma} \Sigma^{\dagger} \xi_{\nu \mu} \Sigma \xi_{\sigma \mu} \Sigma^{\dagger}\right)+$ p.c. \\
81 & $\operatorname{Str}\left(\xi_{\mu \nu} \Sigma \xi_{\mu \sigma} \Sigma^{\dagger}\right) \operatorname{Str}\left(\xi_{\nu \mu} \Sigma \xi_{\sigma \mu} \Sigma^{\dagger}\right)+$ p.c. \\
\hline \hline
\end{tabular}

TABLE XXIII: $\mathcal{O}\left(a^{4}\right)$ operators in the staggered chiral Lagrangian arising from two insertions of $S_{6}^{F F(B)}$ operators. The indices $\mu$ and $\nu$ are separately summed, with the constraint that $\mu \neq \nu$. 


\begin{tabular}{l|cc}
\hline \hline Generic Op. & {$[V, A \times P]$} & {$[V, A \times T]$} \\
\hline 115 & $i \operatorname{Str}\left(\left[\ell_{\mu}, \xi_{5}\right] \xi_{5} \Sigma D_{\mu} \Sigma^{\dagger}\right)+$ p.c. & $i \operatorname{Str}\left(\left[\ell_{\mu}, \xi_{\nu \rho}\right] \xi_{\rho \nu} \Sigma D_{\mu} \Sigma^{\dagger}\right)+p . c$. \\
117 & $i \operatorname{Str}\left(\left[\ell_{\mu}, \xi_{5}\right] \Sigma \xi_{5} D_{\mu} \Sigma^{\dagger}\right)+$ p.c. & $i \operatorname{Str}\left(\left[\ell_{\mu}, \xi_{\nu \rho}\right] \Sigma \xi_{\rho \nu} D_{\mu} \Sigma^{\dagger}\right)+p . c$. \\
\hline \hline
\end{tabular}

\begin{tabular}{l|cc}
\hline \hline Generic Op. & {$[S, P \times V]$} & {$[S, P \times A]$} \\
\hline 119 & $i \operatorname{Str}\left(\left[r_{\mu} \xi_{\nu}-\xi_{\nu} \ell_{\mu}\right] \Sigma \xi_{\nu} D_{\mu} \Sigma\right)+$ p.c. & $i \operatorname{Str}\left(\left[r_{\mu} \xi_{\nu 5}-\xi_{\nu 5} \ell_{\mu}\right] \Sigma \xi_{5 \nu} D_{\mu} \Sigma\right)+$ p.c. \\
121 & $i \operatorname{Str}\left(\left[r_{\mu} \xi_{\nu}-\xi_{\nu} \ell_{\mu}\right] \Sigma\right) \operatorname{Str}\left(\xi_{\nu} D_{\mu} \Sigma\right)+$ p.c. & $i \operatorname{Str}\left(\left[r_{\mu} \xi_{\nu 5}-\xi_{\nu 5} \ell_{\mu}\right] \Sigma\right) \operatorname{Str}\left(\xi_{5 \nu} D_{\mu} \Sigma\right)+p . c$. \\
123 & $i \operatorname{Str}\left(\left[r_{\mu} \xi_{\nu}-\xi_{\nu} \ell_{\mu}\right] \xi_{\nu} \Sigma^{\dagger} D_{\mu} \Sigma\right)+$ p.c. & $i \operatorname{Str}\left(\left[r_{\mu} \xi_{\nu 5}-\xi_{\nu 5} \ell_{\mu}\right] \xi_{5 \nu} \Sigma^{\dagger} D_{\mu} \Sigma\right)+$ p.c. \\
125 & $i \operatorname{Str}\left(\left[r_{\mu} \xi_{\nu}-\xi_{\nu} \ell_{\mu}\right] \Sigma\right) \operatorname{Str}\left(\xi_{\nu} D_{\mu} \Sigma^{\dagger}\right)+$ p.c. & $i \operatorname{Str}\left(\left[r_{\mu} \xi_{\nu 5}-\xi_{\nu 5} \ell_{\mu}\right] \Sigma\right) \operatorname{Str}\left(\xi_{5 \nu} D_{\mu} \Sigma^{\dagger}\right)+$ p.c. \\
\hline \hline
\end{tabular}

TABLE XXIV: $\mathcal{O}\left(a^{2} p^{2}\right)$ operators proportional to sources, arising from single insertions of $S_{6}^{F F(A)}$ operators.

\begin{tabular}{l|c}
\hline \hline Generic Op. & {$\left[V_{\mu}, A_{\mu} \times T_{\mu}\right]$} \\
\hline 115 & $i \operatorname{Str}\left(\left[\ell_{\mu}, \xi_{\mu \nu}\right] \xi_{\nu \mu} \Sigma D_{\mu} \Sigma^{\dagger}\right)+$ p.c. \\
117 & $i \operatorname{Str}\left(\left[\ell_{\mu}, \xi_{\mu \nu}\right] \Sigma \xi_{\nu \mu} D_{\mu} \Sigma^{\dagger}\right)+$ p.c. \\
118 & $i \operatorname{Str}\left(\left[\ell_{\mu}, \xi_{\mu \nu}\right] \Sigma D_{\mu} \Sigma^{\dagger} \Sigma \xi_{\nu \mu} \Sigma^{\dagger}\right)+$ p.c. \\
\hline \hline
\end{tabular}

\begin{tabular}{l|cc}
\hline \hline Generic Op. & {$\left[T_{\mu} \times V_{\mu}\right]$} & {$\left[T_{\mu} \times A_{\mu}\right]$} \\
\hline 123 & $i \operatorname{Str}\left(\left[r_{\mu} \xi_{\mu}-\xi_{\mu} \ell_{\mu}\right] \xi_{\mu} \Sigma^{\dagger} D_{\mu} \Sigma\right)+$ p.c. & $i \operatorname{Str}\left(\left[r_{\mu} \xi_{\mu 5}-\xi_{\mu 5} \ell_{\mu}\right] \xi_{5 \mu} \Sigma^{\dagger} D_{\mu} \Sigma\right)+p . c$. \\
125 & $i \operatorname{Str}\left(\left[r_{\mu} \xi_{\mu}-\xi_{\mu} \ell_{\mu}\right] \Sigma\right) \operatorname{Str}\left(\xi_{\mu} D_{\mu} \Sigma^{\dagger}\right)+$ p.c. & $i \operatorname{Str}\left(\left[r_{\mu} \xi_{\mu 5}-\xi_{\mu 5} \ell_{\mu}\right] \Sigma\right) \operatorname{Str}\left(\xi_{5 \mu} D_{\mu} \Sigma^{\dagger}\right)+p . c$. \\
126 & $i \operatorname{Str}\left(\left[r_{\mu} \xi_{\mu}-\xi_{\mu} \ell_{\mu}\right] D_{\mu} \Sigma\right) \operatorname{Str}\left(\Sigma^{\dagger} \xi_{\mu}\right)+$ p.c. & $i \operatorname{Str}\left(\left[r_{\mu} \xi_{\mu 5}-\xi_{\mu 5} \ell_{\mu}\right] D_{\mu} \Sigma\right) \operatorname{Str}\left(\Sigma^{\dagger} \xi_{5 \mu}\right)+p . c$. \\
\hline \hline
\end{tabular}

TABLE XXV: $\mathcal{O}\left(a^{2} p^{2}\right)$ operators proportional to sources, arising from single insertions of $S_{6}^{F F(B)}$ operators. Indices are independently summed, with the constraint $\mu \neq \nu$. 